\title{
Expression and function of PSD-93 isoforms in hippocampal synapses
}

\author{
Dissertation \\ for the award of the degree \\ Doctor rerum naturalium (Dr. rer. nat.) \\ Division of Mathematics and Natural Sciences \\ of the Georg-August-Universität Göttingen \\ submitted by \\ Juliane Marie Krüger \\ from Lüdenscheid, Germany
}

Göttingen, June 2010 
Dr. Dr. Oliver Schlüter (Reviewer)

Molecular Neurobiology, European Neuroscience Institute Göttingen

Prof. Dr. Nils Brose (Reviewer)

Molecular Neurobiology, Max Planck Institute of Experimental Medicine

Dr. Takeshi Sakaba

Department of Membrane Biophysics, Max Planck Institute for Biophysical Chemistry

Day of oral examination: August $9^{\text {th }}, 2010$ 
I hereby declare that I prepared the PhD thesis "Expression and function of PSD-93 isoforms in hippocampal synapses" on my own and with no other sources and aids than quoted.

Juliane Marie Krüger 


\begin{abstract}
Changes in the strength of synaptic transmission are thought to underlie information processing in the brain. At excitatory synapses in the hippocampus, synaptic strength is determined by the number of AMPA receptors (AMPARs) at the postsynaptic density (PSD). PSD-95 is the prototypical scaffolding protein in the PSD, and has been proposed to be important for synaptic AMPAR function. PSD-95 belongs to the PSD-MAGUK protein family that includes SAP97, SAP102 and PSD-93. PSD-MAGUKs share a common domain structure, but they exist as different isoforms with distinct N-terminal amino acid sequences. These N-termini have previously been shown to govern the function of PSD-95 and SAP97 isoforms, implying selective roles in modulating synaptic strength. PSD-93 has six alternative $\mathrm{N}$-termini, including $\alpha_{1}, \alpha_{2}$, and $\beta$, which are similar to the $\alpha$ - and $\beta$-isoforms of PSD-95 and SAP97, and three unique ones, which have not been analyzed so far.

In this thesis, I analyzed the expression pattern of PSD-93 isoforms in the hippocampus and characterized their function by electrophysiology. Using mass spectrometry and western blotting, PSD-93 $\beta, \gamma_{1}, \delta$, and one or both $\alpha$-isoforms of PSD-93 were detected in the hippocampus. The $\alpha$-isoforms were the most abundant of all. Gain-of-function studies in CA1 pyramidal cells of hippocampal slice cultures demonstrated that only PSD-93 $\delta$ and $\epsilon$ increased AMPAR EPSCs by $50-100 \%$, similar to the $\alpha$-isoforms of PSD95 and SAP97. Although the $\alpha$-N-terminal exon of PSD-93 in principle behaved like a classical $\alpha$-isoform, an inhibitory domain was discovered that blocked the positive action of endogenous PSD- $93 \alpha_{1 / 2}$ on AMPARs. Consistent with this and the fact that PSD-93 $\delta$ and $\epsilon$ make up at most $11 \%$ of PSD-95, knockdown of PSD-95, but not PSD-93, affected basal synaptic transmission. Activity manipulations revealed differential effects of the individual isoforms, and provide evidence that the activity-dependence, and potentially the role in long-term plasticity, may be governed by several PSD-MAGUKs, including the $\beta$-isoforms and PSD-93 .
\end{abstract}




\section{Acknowledgements}

First of all, I am very grateful to my supervisor, Dr. Dr. Oliver Schlüter, for his trust in my abilities. I was completely new to wet-bench sciences, and I was lucky to be taught a variety of techniques. Thank you for giving me a lot of freedom to develop my own ideas and to establish new approaches in the lab. Most of all, thank you for your enthusiasm for science, for sharing ideas and knowledge, and for your unbeaten optimism.

I thank Prof. Nils Brose and Dr. Takeshi Sakaba for being part of my thesis committee, for the time they devoted to this project, and for the discussions we had during our meetings.

I thank my collaborators, Monika Raabe and Dr. Henning Urlaub for the mass spectrometric analysis and the discussions. I am grateful to the AGCT DNA Core Facility for providing high-quality oligonucleotides and for the timely sequencing. I want to thank Dr. Judith Stegmüller, Prof. Nils Brose and Dr. Hiroshi Kawabe, Dr. David Bredt, Dr. Mark Leyland, and Dr. Juan Lerma for sharing antibodies and drugs.

It was a pleasure to be able to participate in the lab seminars of the Brose department, and to share ideas and protocols with the members of the lab. Thank you all for making me feel at home! Special thanks to Dr. Sonja Wojcik for sharing her ES cell protocol which enabled me to successfully generate one conditional knock-out and one knock-in mouse line.

I was very lucky to be surrounded by great people in the lab. Thank you for sharing excitement and frustration, successes and failures at the bench. Martina, thank you for preparing and taking care of the neuronal cultures, and for working together for more than three years. Sandra, thanks for your amazing viruses, your help with plasmid preps, the genotyping, and of course for the cakes and ice cream sessions. I don't know what we would do without our TAs! Yanling, thanks for sharing the setup with me, and for a nice time in Geneva. Huang, you are very gifted with programming, and I hope that you keep IGOR alive! 
Ania, Derya, and Stéph, thank you so much for your friendship! I am grateful for all your comforting words, for your hugs, and for all the laughter. Don't forget: We can do it! I will always remember our amazing trips to Prague and Istanbul, and I will miss our Wednesday evenings!

Many thanks to all the students in the lab, especially to Georg Raiser, Carina Hamann, and Oleksandr Korolov, for their commitment and good work.

I am extremely grateful to my Mom and my Dad for their love, support, and encouragement - in all its forms - throughout my life.

I dedicate this thesis to my wonderful husband Christof. Thank you for being there for me whenever I needed you, and for giving me the freedom and time I needed to do my research. Thank you for your faith in me, for your emotional support, and your unconditional love during all these years! 


\section{Contents}

List of Figures $\quad$ xiii

$\begin{array}{ll}\text { List of Tables } & \text { XV }\end{array}$

Glossary $\quad$ xvii

1 Introduction 1

1.1 The hippocampus as a model system . . . . . . . . . . . 2

1.2 The postsynaptic receptor complex of glutamatergic synapses . . . . . . 3

$1.2 .1 \quad$ NMDA receptors . . . . . . . . . . . . . . . . . . 4

1.2 .2 AMPA receptors . . . . . . . . . . . . . . . 4

1.2.3 NMDAR-dependent plasticity . . . . . . . . . . . . . 5

1.2 .4 PSD-MAGUKs . . . . . . . . . . . . . 6

1.2.4.1 Alternative N-terminal domains . . . . . . . . 7

1.2.4.2 Differential function of the PSD-MAGUKs and their isoforms . . . . . . . . . . . . . 8

1.3 Scope of this thesis . . . . . . . . . . . . . . 11

2 Materials and methods $\quad 13$

2.1 Molecular Biology . . . . . . . . . . . . . . . . . . . . 13

2.1 .1 Plasmid vectors . . . . . . . . . . . . . . . 13

2.1 .2 Bacterial strains . . . . . . . . . . . . . . . . 14

2.1.3 Oligonucleotides . . . . . . . . . . . . . . . . 14

2.1 .4 Kits . . . . . . . . . . . . . . . . . 15

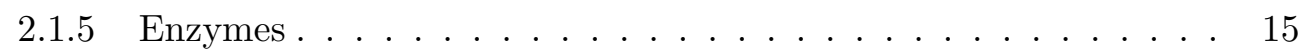

2.1.6 High-quality plasmid preparation . . . . . . . . . . . 15 
2.1.7 Polymerase chain reaction $(\mathrm{PCR}) \ldots \ldots \ldots \ldots$

2.1 .8 DNA constructs . . . . . . . . . . . . . . . . 17

2.1.8.1 PSD-93 knockdown constructs . . . . . . . . . 17

2.1.8.2 PSD-93 overexpression . . . . . . . . . . . 17

2.1.8.3 Replacement of PSD-95 with PSD-93 isoforms . . . . . 18

2.1.8.4 Chimeric constructs . . . . . . . . . . . . . . 19

2.1 .9 Semi-quantitative RT-PCR . . . . . . . . . . . . 20

2.1.9.1 RNA isolation from tissue . . . . . . . . . . 20

2.1.9.2 Reverse Transcription . . . . . . . . . . . . . . . 21

2.1.9.3 Polymerase chain reaction on cDNA . . . . . . . . 21

2.2 Cell culture techniques . . . . . . . . . . . . . . . . . . . . . . 21

2.2.1 HEK293T cell culture . . . . . . . . . . . . . . . . . . 22

2.2.1.1 Transfection of HEK293T cells . . . . . . . . . . 22

2.2.1.2 Lentivirus production . . . . . . . . . . . . 22

$2.2 .2 \quad$ Dissociated hippocampal cultures . . . . . . . . . . . . . . 23

2.2.2.1 Infection of dissociated cultures . . . . . . . . . 23

2.2.3 Organotypic hippocampal slice cultures . . . . . . . . . . . 23

2.2.3.1 Virus injection into slice cultures . . . . . . . . . . . 24

2.3 PSD-93 knock-out strain and genotyping . . . . . . . . . . . . . 25

2.4 Protein-Biochemistry . . . . . . . . . . . . . . . . . . 25

2.4 .1 Antibodies . . . . . . . . . . . . . . . 25

2.4.2 Sample preparation: HEK293T cells . . . . . . . . . . . . 25

2.4.3 Sample preparation: dissociated cultures . . . . . . . . . . . 27

2.4.4 Sample preparation: tissue . . . . . . . . . . . . . 27

2.4.5 Protein concentration with BCA assay . . . . . . . . . . . 28

2.4 .6 SDS-PAGE and western blot . . . . . . . . . . . . 28

2.4 .7 Immunoprecipitation (IP) . . . . . . . . . . . . . . . . . . . 29

2.4 .7 .1 IP from tissue . . . . . . . . . . . . . . . 29

2.4.7.2 IP from dissociated cultures . . . . . . . . . . . 30

2.5 Identification of PSD-93 isoforms by mass spectrometry . . . . . . . . 31

2.6 Electrophysiology . . . . . . . . . . . . . . . . . . . . . . . . . . . . . 32

2.6 .1 Equipment . . . . . . . . . . . . . . . . 32

2.6 .2 Data acquisition . . . . . . . . . . . . . . . 32 
2.6 .3 Pharmacology . . . . . . . . . . . . . . . . 33

2.6 .4 Statistical analysis . . . . . . . . . . . . . . . 33

2.7 Software and web tools . . . . . . . . . . . . . . . 34

3 Expression of PSD-93 in the hippocampus 35

3.1 Developmental profile . . . . . . . . . . . . . . 35

3.2 PSD-93 is alternatively spliced to generate multiple transcripts . . . . . 37

3.2 .1 N-terminal isoforms . . . . . . . . . . . . . . 39

3.2 .2 Splicing at the C-terminus . . . . . . . . . . . . 40

3.3 Tools to study PSD-93 expression and function . . . . . . . . . . . . . 43

3.3.1 Knockdown constructs for PSD-93 . . . . . . . . . . 44

3.3 .2 Antibody characterization . . . . . . . . . . . . . 46

3.3.2.1 Monoclonal PSD-93 antibody . . . . . . . . . . 46

3.3.2.2 Polyclonal PSD-93 antibody . . . . . . . . . . 47

3.3.2.3 $\alpha / \beta$-MAGUK antibody . . . . . . . . . . 48

3.3.3 Identification of PSD-93 isoforms expressed in the hippocampus 51

3.3.3.1 Analyzing the molecular weight of the isoforms . . . . 51

3.3.3.2 Traces of PSD-93 $\delta$ are expressed in the hippocampus . 53

3.3.4 Quantification of PSD-93 and PSD-95 . . . . . . . . 53

3.4 PSD-93 and PSD-95 interact in vivo . . . . . . . . . . . . . . 55

3.5 Analysis of PSD-93 by mass spectrometry . . . . . . . . . . . 58

3.5 .1 Identification of PSD-93 isoforms . . . . . . . . . . . 59

3.5.2 Possible interaction partners of PSD-93 . . . . . . . . . 63

4 The role of PSD-93 in basal synaptic transmission $\quad 67$

4.1 Knockdown of PSD-93 does not affect basal synaptic transmission . . . 69

4.2 Overexpression of PSD-93 isoforms shows differential effects . . . . . . . 69

4.3 Analyzing the molecular structure of PSD-93 . . . . . . . . . . 77

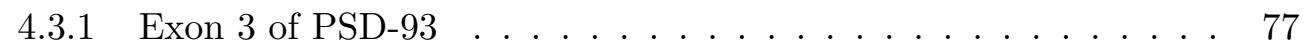

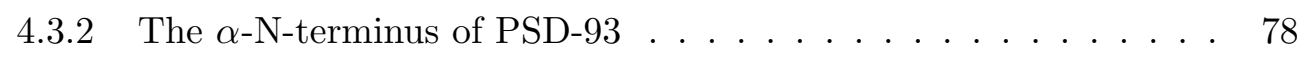

4.4 All isoforms of PSD-93 can compensate for the acute loss of PSD-95 . . 82

4.5 Activity manipulations show distinct properties of the PSD-93 isoforms 86 
$\begin{array}{lll}5 & \text { Discussion } & 91\end{array}$

5.1 Relative abundance of PSD-93 isoforms in the hippocampus . . . . . . . 92

5.2 The PSD-93 $\alpha$-isoforms behave $\alpha$-atypical . . . . . . . . . . . . . . . 94

$5.3 \quad$ PSD-93 $\delta$ affects a conductance other than NMDARs . . . . . . . . . . . 96

5.4 PSD-93 has no role in basal synaptic transmission . . . . . . . . . . 98

5.5 Activity dependence is not specific for the L27 domain . . . . . . . . . . 99

5.6 Conclusions and Outlook . . . . . . . . . . . . . . . . . . 99

$\begin{array}{ll}\text { References } & 103\end{array}$

$\begin{array}{lr}\text { Curriculum Vitae } & 120\end{array}$ 


\section{List of Figures}

1.1 Pyramidal neuron in CA1. . . . . . . . . . . . . . . . 3

1.2 General structure of the PSD-MAGUKs and their binding partners, using PSD-93 as an example. . . . . . . . . . . . . . 7

3.1 Developmental profile of PSD-MAGUKs and glutamate receptor subunits. 36

3.2 Exon-intron structure of PSD-93. . . . . . . . . . . . . . . . 39

3.3 The mRNA of the different PSD-93 isoforms is present in many brain regions, but their abundance differs between tissues as detected by semiquantitative RT-PCR. . . . . . . . . . . . . . . . . . . . 41

3.4 Splice variants between SH3 and GK domain. . . . . . . . . . . . . . 42

3.5 Lentiviral transduction of cells to drive shRNA expression. . . . . . . . . 45

3.6 Knockdown constructs for PSD-93 have differential efficiency in mouse

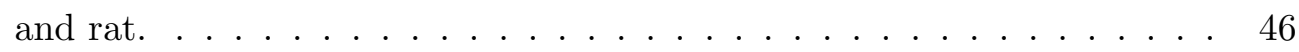

3.7 Characterization of the monoclonal antibody for PSD-93. . . . . . . 47

3.8 Characterization of the polyclonal antibody for PSD-93. . . . . . . . . 48

3.9 At the N-terminus of the PSD-MAGUKs there are conserved regions. . . 49

$3.10 \alpha$-SAP97 detects only the $\alpha$ - and $\beta$-isoforms of PSD-93. . . . . . . . . 50

$3.11 \alpha$-SAP97 has a different affinity for PSD-95, PSD-93 and SAP97. . . . 51

3.12 Expressing untagged PSD-93 shows that PSD-93 $\beta$, PSD-93 $\alpha_{1 / 2}$, PSD$93 \gamma_{1}$ are expressed in the hippocampus. . . . . . . . . . . . . 52

3.13 PSD-93 $\delta$ only makes up at most 5\% of PSD-93 in the hippocampus. . . 54

3.14 Quantification of PSD-93 and PSD-95 in the hippocampus. . . . . . . . 55

3.15 PSD-93 $\alpha_{1 / 2}$ and PSD-95 interact in vivo. . . . . . . . . . . . . 57

3.16 Immunoprecipitation of PSD-93 from hippocampal tissue of PSD-93 WT and $\mathrm{KO}$ mice. . . . . . . . . . . . . . . . . . . . . . . 64 
3.17 Classification of proteins that co-immunoprecipitated with PSD-93. . . . 65

4.1 Simultaneous dual whole-cell patch-clamp recordings of hippocampal slice cultures. . . . . . . . . . . . . . . . . . . . 68

4.2 Knockdown of PSD-93 with shRNAs does not affect AMPAR or NMDAR

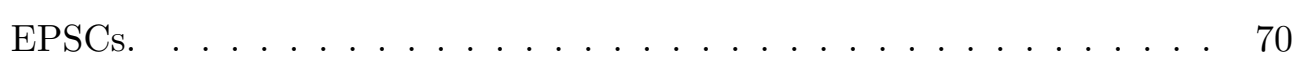

4.3 Overexpressing the $\alpha$-isoforms of PSD-93 does not increase AMPAR

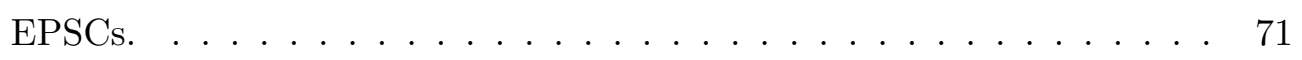

4.4 Overexpressing the non- $\alpha$-isoforms of PSD-93 has differential effects on basal synaptic transmission. . . . . . . . . . . . . . 72

4.5 Overexpressing the $\delta$-isoform increases not only AMPAR EPSCs, but also affects a conductance other than NMDARs. . . . . . . . . . 74

4.6 Exon 3 does not immediately affect basal synaptic transmission. . . . . 78

4.7 Exon 1 of PSD-93 $\alpha_{2}$ is functional and increases AMPAR EPSCs. . . . . 80

4.8 The full N-terminus of PSD-95 $\alpha$, but not exon 1, can convert PSD-93 into a classical $\alpha$-isoform. . . . . . . . . . . . . . . . . 81

4.9 PSD-93 can rescue the acute loss of PSD-95. . . . . . . . . . . . . 84

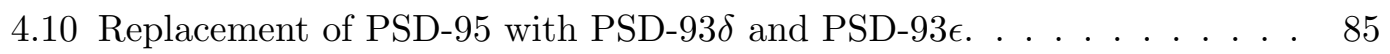

4.11 PSD-93 $\alpha_{1}$, PSD-93 $\alpha_{2}$, and PSD-93 $\gamma_{1}$ do not show activity-dependent

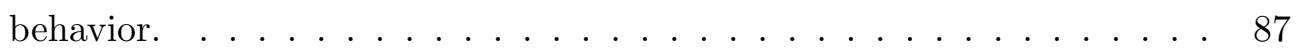

4.12 Activity-dependent increase of AMPAR EPSCs when overexpressing PSD$93 \beta$ is NMDAR-dependent. . . . . . . . . . . . . . 89

4.13 PSD-93 $\delta$ and possibly PSD-93 $\epsilon$ are affected by activity manipulations in the culture dish. . . . . . . . . . . . . . . . 90 


\section{List of Tables}

2.1 Oligonucleotides used in this study . . . . . . . . . . . . . . . . 14

2.2 Enzymes used in this study for molecular cloning. . . . . . . . . . . . 16

2.3 Antibodies used in this study for western blotting. . . . . . . . . . . 26

3.1 Nomenclature of the N-terminal splice variants of PSD-93. . . . . . . . 38

3.2 N-terminal peptides generated by trypsin digest. . . . . . . . . . . . . 60

3.3 N-terminal peptides generated by chymotrypsin digest. . . . . . . . . . 62

5.1 Summary of the expression and function of PSD-93 isoforms. . . . . . 100 


\section{Glossary}

\begin{tabular}{|c|c|}
\hline ACSF & Artificial cerebrospinal fluid \\
\hline AMPAR & $\alpha$-amino-3-hydroxyl-5-methyl-4-isoxazole-propionate (AMPA) receptor \\
\hline APP & Amyloid precursor protein \\
\hline $\mathrm{BIC}$ & Bicuculline \\
\hline CA1 & Cornu ammonis region 1 \\
\hline CA3 & Cornu ammonis region 3 \\
\hline CaMKII & Calcium-calmodulin-dependent protein kinase II \\
\hline CMV & Cytomegalovirus \\
\hline DIV & Day in vitro \\
\hline E. coli & Escherichia coli \\
\hline EPSC & Excitatory postsynaptic current \\
\hline EST & Expressed sequence tag \\
\hline GIRK & G protein-coupled inwardly-rectifying potassium channel \\
\hline GK & Guanylate kinase \\
\hline HB & Homogenization buffer \\
\hline IP & Immunoprecipitation \\
\hline IRES & Internal ribosomal entry site \\
\hline KAR & Kainate receptor \\
\hline KO & Knockout \\
\hline LTD & Long-term depression \\
\hline LTP & Long-term potentiation \\
\hline MAGUK & Membrane associated guanylate kinase \\
\hline mEPSC & Miniature excitatory postsynaptic current \\
\hline
\end{tabular}




\begin{tabular}{ll} 
mGluR & Metabotropic glutamate receptor \\
MS & Mass spectrometry \\
ms & Mouse \\
NMDAR & Number of experiments \\
PX & N-methyl-D-aspartic acid (NMDA) receptor \\
PCR & Postnatal day $X$ \\
PDZ & Polymerase chain reaction \\
Pol III & Postsynaptic density-95/Discs large/zona occludens-1 \\
PPF & Polymerase III \\
PPR & Paired pulse facilitation \\
PSD & Paired pulse ratio \\
rb & Postsynaptic density \\
RNAi & Rabbit \\
SDS-PAGE & RNA interference \\
SEM & SDS-Polyacrylamid gel electrophoresis \\
SH3 & Standard error of the mean \\
shRNA & Src homology 3 \\
SK channel & Short-hairpin RNA conductance Ca \\
TARP & Transmembrane AMPAR regulatory proteins \\
\hline
\end{tabular}




\section{1}

\section{Introduction}

Neurons, the key players in the nervous system, are capable of generating electrical signals in response to certain stimuli. The $10^{11}$ neurons in the human brain form interacting networks which enable them to communicate with each other and to perform complex computations, crucial for brain function. The fundamental means of communication is the action potential. The action potential is a rapid reversal of the membrane potential of the neuron. Action potentials are mediated by voltage-gated ion channels in the plasma membrane, and the action potential can be propagated from the cell body along the axon to the presynaptic terminal of a synapse. The synapse, point of communication between two neurons, is the region where the plasma membranes of two cells are in close proximity and equipped with specific proteins. One type of synapse is the chemical synapse, which has an orientation and consists of a presynaptic and a postsynaptic site. At the presynaptic terminal, the action potential triggers the release of a chemical substance, the neurotransmitter. The neurotransmitter traverses the synaptic cleft to bind to receptors on the postsynaptic site. On the postsynaptic site, the activation of the receptor allows ions to pass, and leads to a change in the excitability of the cell, thus converting the chemical signal back into an electrical one. If the depolarization caused in the postsynaptic cell body reaches a certain threshold, a new action potential is generated.

It is already quite impressive that neurons can communicate with each other via synapses. But it is even more stunning that these synaptic connections are not fixed in strength, but rather undergo use-dependent modifications which lead to either strengthening or weakening of the connections. These modifications of synaptic efficiency, re- 


\section{INTRODUCTION}

ferred to as synaptic plasticity, are assumed to be the cellular mechanism of learning and memory (Bliss and Lømo 1973; Hebb 1949; Malenka and Bear 2004; Milner et al. 1998). In fact, the synapse is thought to be the actual site of memory storage. Each neuron contains thousands of synapses, which function primarily autonomously.

The neurotransmitter glutamate mediates the excitatory synaptic transmission in the central nervous system. Excitatory synaptic transmission is the active form of information transport since activation of glutamatergic receptors leads to a depolarization of the cell, and eventually to the generation of an action potential. In contrast, the neurotransmitters glycine and GABA lead to an inhibitory response in the postsynaptic cell, thereby modulating the effect of glutamate receptor activation. Inhibitory transmission is important to organize brain microcircuits by generating rhythmic neuronal activity (Freund 2003; McBain and Fisahn 2001).

\subsection{The hippocampus as a model system}

The best-studied forms of synaptic plasticity are long-term potentiation (LTP) and long-term depression (LTD) of excitatory synaptic transmission (Bredt and Nicoll 2003; Malinow and Malenka 2002). Although such forms of plasticity have been observed at many synapses in the central nervous system, the hippocampus has evolved as a model system for plasticity. First, the hippocampus plays an important role in episodic memory and spatial learning (Andersen et al. 2007; Kesner and Hopkins 2006; Milner and Penfield 1955; Rogers and Kesner 2006). Second, the hippocampus possesses a laminar structure which makes it particularly suitable for electrophysiological recordings.

The hippocampal formation was classically described as a trisynaptic circuit, with the entorhinal cortex projecting to the dentate gyrus, which connects to cornu ammonis region 3 (CA3), and which in turn projects to cornu ammonis region 1 (CA1) (Andersen et al. 1971). Although it is now known that the connectivity is more complex, e.g. the entorhinal cortex not only projects to the dentate gyrus but also to CA3 and CA1 (Amaral and Witter 1989), the basic directionality is beyond dispute. The hippocampus can be cultured as organotypic slices (Gähwiler 1981; Stoppini et al. 1991). Facilitated by the laminar structure, the slices in culture keep their connectivity and develop with a similar time course as in vivo (Muller et al. 1993; Simoni et al. 2003). 
About $90 \%$ of the cells in the hippocampus are excitatory cells. Pyramidal cells reside in $\mathrm{CA} 3$ and $\mathrm{CA} 1$, and granule neurons in the dentate gyrus. Pyramidal neurons have a characteristic triangular shape, as shown in Figure 1.1. They possess multiple dendrites but only a single axon. Only $10 \%$ of the cells in the hippocampus are local inhibitory interneurons.

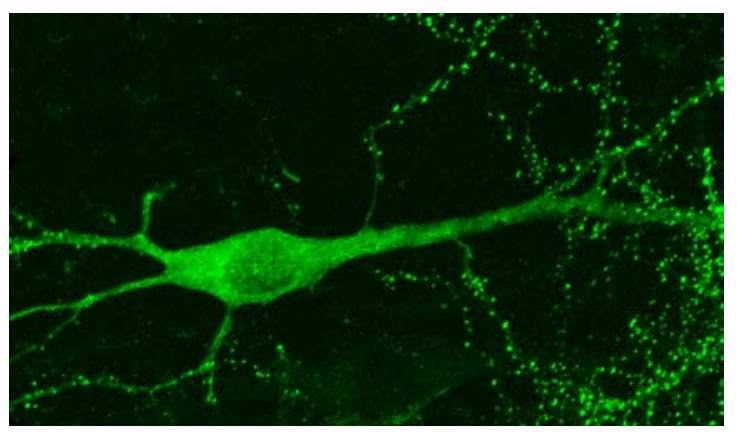

Figure 1.1: Pyramidal neuron in CA1. Example of a CA1 pyramidal cell in a slice culture overexpressing PSD-95 as a GFP-fusion protein.

The excitatory projection from CA3 onto CA1 pyramidal cells formed by the Schaffer collateral axons is the most-studied synapse and is believed to be representative for many other synapses in the brain (Andersen et al. 2007; Shepherd 2003). The present study focusses on the postsynaptic site of this CA3-CA1 synapse.

\subsection{The postsynaptic receptor complex of glutamatergic synapses}

Glutamate is the major excitatory neurotransmitter in the hippocampus. It activates three ionotropic glutamate receptors which have been termed after their selective agonists AMPA, NMDA, and kainate. AMPA receptors (AMPARs), NMDA receptors (NMDARs), as well as kainate receptors (KARs) are assembled as heteromeric complexes of four subunits which form an ion channel. Experimentally, the ion flux through these channels is measured as the excitatory postsynaptic current (EPSC). For this, the cell is clamped at a fixed membrane potential and inhibitory transmission is pharmacologically blocked. In addition to these ionotropic receptors, glutamate also activates G protein-coupled metabotropic receptors (mGluRs), transmembrane receptors which do not form ion channels but induce intracellular signaling cascades upon activation. 


\section{INTRODUCTION}

Synaptic AMPARs, NMDARs, and a subset of mGluRs are found on spines, mushroom-shaped protrusions of the dendrite, in the postsynaptic density (PSD). The PSD is an electron-dense region as viewed by electron microscopy which is characteristic for excitatory synapses. It is found at the spine head and is about half a micron in width, and 25-50nm thick. Apart from receptors, it harbors scaffolding and cell adhesion proteins as well as downstream signaling molecules.

\subsubsection{NMDA receptors}

NMDARs form a tetrameric complex which in the hippocampus mainly consists of the subunits NR1, NR2A, and NR2B (Andersen et al. 2000; Laurie et al. 1997; Wenzel et al. 1997). The incorporation of NR1 is required for the formation of a functional receptor, since it provides the binding site for the coagonist glycine or D-serine (Wenthold et al. 2003). The NR2A and NR2B subunits contain the binding site for the agonist glutamate (Laube et al. 1997).

In the hippocampus, NR2B is the NR2 subunit which is predominantly expressed in early postnatal development. During maturation, more NR2A subunits are added to the synapse, eventually outnumbering NR2B subunits. This is called the NR2B to NR2A developmental switch (Liu et al. 2004). The NR2 subunit also defines the channel's kinetics, and the presence of NR2A leads to a 3-4 times faster decay time (Monyer et al. 1994).

Importantly, NMDARs are blocked by extracellular $\mathrm{Mg}^{2+}$ ions and only open when the postsynaptic neuron is depolarized. Thus, NMDARs act as coincidence detectors of pre- and postsynaptic activity. NMDA receptors have a high calcium permeability and slow activation and deactivation kinetics, which result from the fact that glutamate stays bound to the receptor for a long time (Ascher and Nowak 1988; Lester et al. 1990). Because of the $\mathrm{Mg}^{2+}$ block at resting potential, AMPARs mediate most of the constitutive or basal synaptic transmission.

\subsubsection{AMPA receptors}

AMPARs form tetramers of the subunits GluR1-GluR4. The subunits differ mainly in their intracellular C-termini and thereby bind different interaction partners (Malinow 
and Malenka 2002). GluR2 defines the properties of the AMPAR. GluR2 is posttranslationally edited and this modification renders the channel $\mathrm{Ca}^{2+}$-impermeable. In addition, AMPARs lacking the GluR2 subunit do not pass current at positive membrane potentials because they are more susceptible of being blocked by polyamines. This leads to an inward rectification, experimentally observed when measuring the currentvoltage-relationship of the channel. Apart from its role in synaptic transmission, the subunit composition is also believed to influence the receptor's trafficking (Bredt and Nicoll 2003; Malinow and Malenka 2002).

In the hippocampus, AMPARs are mainly composed of either dimers of GluR1 and GluR2 subunits (termed GluR1/2), or two GluR3 and two GluR2 subunits (termed GluR2/3) (Wenthold et al. 1996). It has been suggested that GluR2/3-containing AMPARs mediate basal synaptic transmission, and are continuously recycled on a 15min timescale, whereas GluR1/2 containing AMPARs are inserted into the synapse during LTP (Hayashi et al. 2000; Passafaro et al. 2001; Shi et al. 2001). However, more recently, Lu et al. (2009) showed with the help of single-cell subunit deletion that in basal conditions, $81 \%$ of the synapses contain GluR $1 / 2$, and only $15 \%$ GluR2/3, and almost all of the extrasynaptic receptors are GluR1/2. The existence of homomeric GluR1 containing receptors and their functional relevance is highly debated $(\mathrm{Lu}$ et al. 2009; Wenthold et al. 1996). While some groups found that calcium-permeable AMPARs, specifically GluR1 homomers, are inserted into the synapse during early LTP (He et al. 2009; Plant et al. 2006), others argue against the existence and necessity of homomeric GluR1s for LTP (Adesnik and Nicoll 2007; Bagal et al. 2005). This demonstrates that the subunit composition of the AMPARs in the hippocampus is still the subject of ongoing research.

\subsubsection{NMDAR-dependent plasticity}

Calcium influx through NMDARs is required to trigger certain forms of plasticity, the so-called NMDAR-dependent forms of plasticity. LTP is observed as an increase in AMPAR-mediated current, and by contrast, LTD leads to a decrease. Although an increase in AMPAR-mediated transmission could in principle result from an increased net current through the receptors present in the synapse, it has been shown that in LTD and LTP the number of AMPARs in the PSD is changed (Collingridge et al. 2004; Malinow and Malenka 2002). Weak activation of NMDARs, and thus only a slight 


\section{INTRODUCTION}

increase in intracellular calcium levels, leads to an activation of protein phosphatases, PP1 and PP2B, that weakens synaptic transmission in a long-term fashion, or LTD. On the other hand, a strong activation of NMDARs and high intracellular calcium levels triggers a Calcium-calmodulin-dependent protein kinase II (CaMKII)-dependent strengthening of synaptic transmission, or LTP. However, which signaling cascades are involved in the weakening and strengthening are just beginning to be understood (Shepherd and Huganir 2007).

A general model has emerged in which LTP and LTD require regulated endo- and exocytosis of AMPARs. However, the detailed molecular events of AMPAR trafficking and stabilization at the synapse remain to be elucidated. It is for instance not clear to what degree lateral diffusion of AMPARs from the dendritic surface to the synapse (Borgdorff and Choquet 2002; Heine et al. 2008; Newpher and Ehlers 2009; Passafaro et al. 2001), and exocytosis from local intracellular pools (Park et al. 2004; Passafaro et al. 2001; Yang et al. 2008) contribute to the expression of LTP.

AMPARs and NMDARs are part of a protein network, and it is believed that these protein interactions play key roles in mediating basal synaptic transmission and the induction and maintenance of synaptic plasticity. One set of proteins identified to play a role in AMPAR trafficking and function is a subfamily of membrane associated guanylate kinases (MAGUKs), the PSD-MAGUKs.

\subsubsection{PSD-MAGUKs}

The proteins chapsyn-110/PSD-93/dLg2, PSD-95/SAP90/dLg4, SAP102/dLg3, and SAP97/dLg1 belong to the family of PSD-MAGUKs. PSD-MAGUKs share a common set of modular protein interaction motifs, including three postsynaptic density-95/Discs large/zona occludens-1 (PDZ) domains, a Src homology 3 (SH3) domain, and an enzymatically inactive guanylate kinase (GK) domain (Kim and Sheng 2004). PDZ domains interact with short amino acid motifs at the C-terminus of certain proteins, such as NR2 subunits (Niethammer et al. 1996). Known interaction partners of the PSD-MAGUKs are illustrated in Figure 1.2, taking PSD-93 as an example, and are reviewed in Kim and Sheng (2004). Despite extensive similarity, the PSD-MAGUKs exist as different isoforms with distinct $\mathrm{N}$-terminal amino acid sequences as a result of alternative promoter usage. 


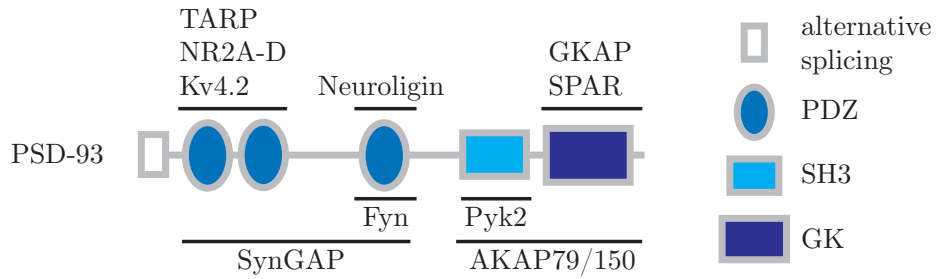

Figure 1.2: General structure of the PSD-MAGUKs and their binding partners, using PSD-93 as an example. The PSD-MAGUKs share a common domain structure in having three PDZ domains, an SH3 and a GK domain. The N-terminus is alternatively spliced. They interact with several proteins through their interaction domains. The proposed site of interaction is indicated by the bars.

\subsubsection{Alternative N-terminal domains}

PSD-95 and SAP97 exist with two different N-terminal domains. The $\alpha$-isoform is identical for PSD-95 and SAP97 (Schlüter et al. 2006), and contains a cysteine doublet (CxC) that has been shown to be palmitoylated (Topinka and Bredt 1998). Palmitoylation is a post-translational modification in which a saturated 16-carbon palmitic acid is conjugated to a cysteine residue of a protein through the formation of a labile thioester bond. Palmitoylation is special among the protein modifications in that it is reversible. Palmitoylation and depalmitoylation happen faster than the half-life of the modified protein, and can be constitutive or dynamically regulated (for a review see Fukata and Fukata 2010). For instance, targeting PSD-95 to the synapse has been shown to depend on palmitoylation, and the palmitoylation is accelerated by glutamate receptor activation (Craven et al. 1999; El-Husseini et al. 2000a, 2002; Topinka and Bredt 1998). However, in addition to a palmitoylated N-terminus, the C-terminal SH3 and/or GK domains are also required for PSD-95's stabilization in the PSD (Xu et al. 2008). Palmitoylation allows PSD-95 to multimerize with itself or with other palmitoylated proteins such as PSD-93 (Hsueh et al. 1997; Kim et al. 1996). This N-terminal homomultimerization is required for PSD-95's effect on basal synaptic transmission (Xu et al. 2008).

The $\beta$-isoforms of PSD-95 and SAP97 have an L27 domain which serves as an additional protein-protein-interaction domain to homomultimerize with itself or to heteromultimerize with other L27 domain-containing proteins, such as CASK (Chetkovich et al. 2002; Lee et al. 2002). Multimerization of the $\beta$-isoforms via the L27 domain 


\section{INTRODUCTION}

might be important for the synaptic function of SAP97 $\beta$ (Nakagawa et al. 2004). However, whether the $\beta$-isoforms are localized to the synapse is debated, as some groups see a synaptic enrichment (Chetkovich et al. 2002; Nakagawa et al. 2004; Rumbaugh et al. 2003), whereas in another study SAP97 $\beta$ was observed both in spines and diffusely in the dendrites and cell bodies (Waites et al. 2009). The observed discrepancies might result from different expression levels as the former studies used transfection which results in much higher expression than the lentiviral-mediated transduction used in the later study.

Only one isoform has been found for SAP102/Dlg3, and it does not possess a palmitoylated N-terminus or an L27 domain. Rather, its N-terminal cysteines have been shown to bind to zinc (El-Husseini et al. 2000c).

PSD-93, on the other hand, exists with six alternative N-terminal domains (Parker et al. 2004). Two $\alpha$-like isoforms contain a cysteine doublet that can be palmitoylated in vivo (El-Husseini et al. 2000c). In sharp contrast to PSD-95, palmitoylation of PSD-93 seems to be dispensable for synaptic targeting (Firestein et al. 2000), although another group proposed that palmitoylation of PSD-93 $\alpha_{1}$ is required for its membrane association (Nada et al. 2003). The third isoform of PSD-93 contains an L27 domain, similar to PSD-95 $\beta$ and SAP97 $\beta$. The remaining three isoforms are unique to PSD-93 (Parker et al. 2004). PSD-93 is alternatively spliced at the C-terminus, and it has a unique insert between PDZ2 and PDZ3 that does not occur in any of the other PSD-MAGUK proteins (Brenman et al. 1996).

\subsubsection{Differential function of the PSD-MAGUKs and their isoforms}

PSD-95 is the prototypical scaffolding protein in the PSD, and has been proposed to be the key in synaptic AMPAR function. Besides, PSD-95 has been suggested to be important for synapse development, as overexpression of PSD-95 accelerates the maturation of excitatory synapses (El-Husseini et al. 2000b). However, genetic evidence for this hypothesis remains elusive. Whereas Migaud et al. (1998) observed no alteration in AMPAR- and NMDAR-mediated basal synaptic transmission and normal synaptic localization of NMDARs in the PSD-95 knockout (KO) animals, Béique et al. (2006) found an increased number of AMPAR-lacking synapses (so-called silent synapses) in the PSD-95 KO mice. In addition, a higher proportion of NR2B-containing NMDARs was observed, indicating deficits in the developmental switch from NR2B to NR2A. 
In the hippocampus, PSD-95 is mainly expressed as the $\alpha$-isoform (Chetkovich et al. 2002). PSD-95 indirectly interacts with AMPARs via transmembrane AMPAR regulatory proteins (TARPs), and this interaction has been suggested to modulate AMPAR trafficking and stability at the synapse (Bats et al. 2007; Chen et al. 2000; Dakoji et al. 2003; Fukata et al. 2005). In line with this model, overexpression of PSD95 enhances AMPAR-mediated transmission (Elias et al. 2006; Nakagawa et al. 2004; Schlüter et al. 2006; Schnell et al. 2002), and occludes LTP (Stein et al. 2003) whereas RNA interference (RNAi)-mediated knockdown of PSD-95 reduces AMPAR-mediated transmission (Elias et al. 2006; Nakagawa et al. 2004; Schlüter et al. 2006). This data prompted the idea that PSD-95 acts as a "slot protein" for the continuous recycling of existing AMPARs to the synapse (Schnell et al. 2002), a concept introduced by Shi et al. (2001). However, contradictory to this direct correlation of PSD-95 and AMPAR number, LTP was enhanced in PSD-95 KO animals and LTD absent (Béïque et al. 2006; Migaud et al. 1998). This indicates that PSD-95 is not directly involved in the recruitment of GluR1-containing AMPARs during LTP. Therefore it has been proposed that PSD-95 acts simultaneously as a signaling scaffold to link NMDAR activation to downstream signaling pathways and as a "gatekeeper" to limit the access of AMPARs to the synapse (Schlüter et al. 2006). In the PSD-95 KO, AMPARs can access the synapse without any checkpoint to pass, since the "gatekeeper" PSD-95 is absent, thus resulting in enhanced LTP.

PSD-95 interacts directly with NMDARs and it was first assumed that this association stabilizes NMDARs in the synapse (Roche et al. 2001). Interestingly, neither overexpression nor knockdown via RNAi alters NMDAR-mediated transmission (Béique and Andrade 2003; Ehrlich and Malinow 2004; Elias et al. 2006; Nakagawa et al. 2004; Schlüter et al. 2006; Schnell et al. 2002; Stein et al. 2003), although one group sees an increase in NMDAR EPSCs following overexpression (Futai et al. 2007).

SAP97 $\beta$ is the only PSD-MAGUK that has been shown to directly bind to AMPARs (Cai et al. 2002; Leonard et al. 1998). Some studies show that SAP97 $\beta$-overexpression can enhance AMPAR-mediated transmission (Nakagawa et al. 2004; Rumbaugh et al. 2003) and partially occlude LTP. This enhancement is thought to depend on the I3 exon, which provides a binding site for the GluR1 binding protein $4.1 \mathrm{~N}$ that links AMPARs to the cytoskeleton (Rumbaugh et al. 2003). However, in other studies, SAP97 $\beta$ overexpression did not affect AMPAR-mediated transmission (Ehrlich and Malinow 


\section{INTRODUCTION}

2004; Schlüter et al. 2006; Schnell et al. 2002), and Waites et al. (2009) even observed a reduction in AMPAR EPSC amplitude.

In particular, different functional roles have been attributed to the $\alpha$ - and $\beta$ isoforms, which are independent of the "backbone" of the protein (e.g. SAP97 or PSD95). The $\alpha$-isoform has been shown to regulate AMPAR responses independently of activity, whereas the effects of the $\beta$-isoform are dependent on activity and CaMKII. Besides, the presence of the $\alpha$-isoform masks the effect of the $\beta$-isoforms on synaptic transmission (Schlüter et al. 2006). SAP97 $\beta$ has been suggested to play a role in the biosynthetic pathway of AMPARs, specifically in the forward trafficking from the Golgi network to the plasma membrane (Sans et al. 2001). Its role as a synaptic scaffold is highly debated (Ehrlich and Malinow 2004; Rumbaugh et al. 2003; Schlüter et al. 2006; Schnell et al. 2002; Waites et al. 2009).

The data on PSD-93 function are rather limited. Its role in synaptic transmission is not clear, and might be dependent on the synapse and brain region under investigation. PSD-93 knockout animals did not show any phenotype in synaptic transmission in the cerebellum or motor behavior (McGee et al. 2001). In cerebellar neurons, synaptic components assembled normally in the knockout animals, suggesting that PSD-93 is not required for synapse development (McGee et al. 2001). On the other hand, in cultured neurons from spinal dorsal horn from PSD-93 KOs, the NR2A and NR2B surface expression was reduced, while the GluR1 expression level was not changed. Also, NMDAR-mediated transmission was altered and NMDAR-dependent persistent pain was reduced in the KO mice (Tao et al. 2003).

Yet another phenotype was observed in the study of Elias et al. (2006), which showed a similar role for PSD-93 and PSD-95 in basal synaptic transmission in the hippocampus. Knockdown of either protein did not affect NMDAR-dependent transmission, but resulted in a $50 \%$ reduction of AMPAR-mediated currents, whereas the overexpression of the $\alpha$-isoform increased AMPAR-mediated currents. Miniature excitatory postsynaptic current (mEPSC) frequency but not amplitude was reduced when either protein was knocked down by RNAi. mEPSCs result from spontaneous fusion events of individual synaptic vesicles. A change in mEPSC amplitude can arise from changes in receptor number or their conductance at the postsynapse. A change in mEPSC frequency can be due to changes in release probability at the presynaptic site, or a change in the number of AMPAR-lacking synapses (silent synapses, see Isaac et al. 
1995). In Elias et al. (2006), the reduction in mEPSC frequency but not amplitude together with the reduction in the amplitude of evoked AMPAR EPSC thus likely result from the silencing of a proportion of synapses, possibly because PSD-93 and PSD-95 are localized to different sets of synapses. The data from the assays performed in the hippocampus led to the assumption that PSD-93 and PSD-95 fulfill the same function, but in different synapses and that in the case of the $\mathrm{KO}$ of one protein, the other family member can compensate. However, it seems unlikely that the proteins will perform the exact same function in their natural setting.

As discussed earlier, it has been shown that the specific function of PSD-95 and SAP97 is solely governed by their N-terminal domains (Schlüter et al. 2006). The observation that PSD-93 and PSD-95 have identical functional properties would suggest that PSD-93 is expressed as an $\alpha$-isoform. However, the study of Schlüter et al. (2006) also implied that neither the $\alpha$ - nor the $\beta$-isoforms of PSD-93 are expressed in the hippocampus. This prompted the question which isoform of PSD-93 can behave $\alpha$-like, and which functional properties are conferred to PSD-93 by the other N-termini.

In the course of this study, a paper was published revisiting some of the data on plasticity in the PSD-93 and PSD-95 knockout animals. LTP has been shown to be enhanced in the PSD-95 KO mice previously, and LTD blocked (Béique et al. 2006; Migaud et al. 1998). However, in the paper of Carlisle et al. (2008) a phenotype for PSD-93 in plasticity was found, for the very first time. In PSD-93 KO mice, a weak stimulation protocol was unable to induce LTP. Thus, PSD-93 KOs have an increased threshold for LTP but normal LTD, showing contrasting roles for the two PSD-MAGUKs. Thus, PSD-93 and PSD-95 most likely couple to different signaling cascades, PSD-93 to facilitators of LTP, and PSD-95 to inhibitors of LTP.

\subsection{Scope of this thesis}

It is not known which isoforms of PSD-93 are expressed in the hippocampus and whether they possess different functional properties. The first aim of this study was to determine which isoforms are present in the hippocampus. Given the importance of maintaining a constant level of synaptic transmission as the basis of long-lasting plasticity, the second aim was to provide a comprehensive analysis of PSD-93 isoforms with respect to their role in basal synaptic transmission and activity manipulations. 


\section{2}

\section{Materials and methods}

\subsection{Molecular Biology}

For the cloning of DNA constructs, standard methods for preparative and analytical digestion, ligation, and electroporation of Escherichia coli (E. coli) were used (Sambrook 2001). Deviations from standard protocols are indicated in the following sections. DNA sequencing was carried out by the AGCT DNA Core Facility of the Max Planck Institute of Experimental Medicine, Göttingen, Germany.

\subsubsection{Plasmid vectors}

The following plasmids were used in this study:

\begin{tabular}{ll}
\hline Plasmid & Reference \\
\hline pBluescriptII SK(-) & Stratagene (Santa Clara, CA, USA) \\
FUGW & Lois et al. (2002) \\
FHUG+W & Schlüter et al. (2006) \\
pSuper & Brummelkamp et al. (2002) \\
pBU6 $^{1}$ & \\
\hline
\end{tabular}

\footnotetext{
${ }^{1}$ pBU6 was generated in the Schlüter lab by amplifying the human U6 promoter (hU6) by PCR and cloning it into the BamHI/EcoRV sites of pBluescriptII SK(-) in which the XhoI site has been eliminated. The primers amplifying hU6 also introduced a 5' EcoRI site and a 3' SmaI site for linearization, as well as the Polymerase III (Pol III) stop signal TTTTT.
} 


\section{MATERIALS AND METHODS}

\subsubsection{Bacterial strains}

Molecular cloning was done using the E. coli strains XL1-Blue (Stratagene, Santa Clara, CA, USA) or TOP10 (Invitrogen, Darmstadt, Germany).

\subsubsection{Oligonucleotides}

The primers used in this study are listed in Table 2.1 and were obtained from SigmaGenosys (Steinheim, Germany) or the AGCT DNA Core Facility of the Max Planck Institute of Experimental Medicine (Göttingen, Germany). The primer ID as designated by the AGCT DNA Core Facility is listed where applicable. In general, a prime in the primer name indicates a reverse primer.

Table 2.1: Oligonucleotides used in this study

\begin{tabular}{lll}
\hline Name & Primer ID & Sequence \\
\hline c93 $\gamma_{1}$ & 12583 & AAGAATTCGCCACCATGTTACCGACTTTCGATATGCAACG \\
c93 $\gamma_{2}$ & 12584 & AAGAATTCGCCACCATGCAACACGCGTTCATTCC \\
c93 $\gamma^{\prime}$ & 12585 & CACGAGGAGAAGGAAGCTTTTGTCAC \\
$93 \beta^{\prime}$ & 12078 & TAGAGCCGGCTTCCTTGAG \\
$93 \alpha_{1}$ & 16484 & CTGAGCTCTCACCACTCAGTGCCTTC \\
$93 \alpha_{2}$ & 16485 & AGCTGCCGCTCGGTCTAGGCTG \\
$93 \delta$ & 16486 & GGGAGGAAGCCTTTCTATGCAG \\
$93 \epsilon$ & 16487 & GCCAACTGGATGTGTGTGAGCCG \\
$93 \gamma$ & 16490 & GTGAAGAAGCTATGCAACATGCGT \\
$93 \gamma^{\prime}$ & 16491 & CGAGTTGCAGTACTGTGCTGG \\
$93{ }^{\prime}$ & 16492 & CGGTGGCCCATAAGGATCAGT \\
$93 \beta$ & 17984 & AAGGCAAATGCCCAGCCCAG \\
$93 \epsilon^{\prime}$ & 18577 & CACAACAGTCTCCAATATGGGTCGC \\
E15 & & AGAAGGGTCACACTAGATGG \\
E16 & 16762 & GTGGAAAGAAAGGAGCGTG \\
E17a & & CCATTCTACAAGAACAAGGAGC \\
E17a' & 16764 & ATCACTGGTTTCCTGCTCAC \\
E17 & 16766 & ATTAGGTGACGACGGTTATGG \\
E17' & 16763 & GAGTCTTTGTTCCATAACCGTC \\
E17b' & 16765 & AGCTACTTTCGCTATCGCTG \\
E18' & 16889 & GCCTCGTGACAGGTTCATAG \\
$93 \alpha_{2} 3 B a m H I '$ & 17057 & GTGGATCCGCTAACTTCTCCTTTGAGGGAATCC \\
& &
\end{tabular}




\begin{tabular}{|c|c|c|}
\hline Name & Primer ID & Sequence \\
\hline $93 \alpha_{2} 5$ EcoRI & 17256 & TCGAATTCCCACCATGATTTGCCACTGCAAAGTTG \\
\hline cB95Esp & 17662 & CGTCTCGCACATCGGTGACGACCCG \\
\hline cB95Esp' & 17657 & CGTCTCATGTGCGGGTTGTCAGTGC \\
\hline cB93Esp & 17658 & CGTCTCTCACATTGGAGATGACCCTG \\
\hline cB93Esp' & 17661 & CGTCTCATGTGAGGATTATCTGTCCCTC \\
\hline $95 \mathrm{~N} 1-9$ & & TCGTCTCTACTATACAGAGACAGTCCATGGTGGGAATTCGATATC \\
\hline 95N1-9' & & TCGTCTCTTAGTGACAACCAAGAAGTATCGATACCAAGATG \\
\hline p93 $\mathrm{N}_{1-20} 1$ & & GTCGTTGATGTTTGGATGCAAGAAATACCGCTACCAAGATG \\
\hline p93N $\mathrm{N}_{1-20} 1^{\prime}$ & & GCAACTTTGCAGTGGCAAATCATGGTGGTCTAGAAAGGGC \\
\hline $\mathrm{p} 93 \mathrm{~N}_{1-20} 2$ & & TCGTCTCTAACAACACTTTGTCGTTGATGTTTGGATGC \\
\hline p93N $\mathrm{N}_{1-20} 2$ & & TCGTCTCTTGTTGGTGCAAGCAACTTTGCAGTGGCAAATC \\
\hline p93-intron & 18576 & GTGCGGAATGTTGTTGTGCAGTGC \\
\hline p93-exon' & 18577 & CACAACAGTCTCCAATATGGGTCGC \\
\hline p93-neo' & & GCCTTCTATCGACTTCTTGACGAG \\
\hline $\operatorname{sh} 93 \mathrm{~d}$ & 15198 & $\begin{array}{l}\text { TCGTCTCAAAGAGATCTTCAATAACAAGCTTGCTTTTTGGA } \\
\text { AAGCTTATCGATACC }\end{array}$ \\
\hline sh93d' & 15199 & $\begin{array}{l}\text { TCGTCTCATCTTGAATCTTCAATAACAAGCTTGCGGGGATC } \\
\text { TGTGGTCTCATAC }\end{array}$ \\
\hline tn93b & 15862 & $\begin{array}{l}\text { CAGCTCGAGGCAGGGAAGTCTTGTATTCTAATGTGCCTTTT } \\
\text { TGATCAAGCTTATCGATACCGTCG }\end{array}$ \\
\hline tn93b' & 15863 & $\begin{array}{l}\text { CAGCTCGAGGCAGGGAAGTCTTGTATTCTAATGTGCCGGTG } \\
\text { TTTCGTCCTTTCCACAAG }\end{array}$ \\
\hline
\end{tabular}

\subsubsection{Kits}

Purification of PCR products or restriction digests was performed using the QIAquick PCR Purification Kit purchased from Qiagen (Hilden, Germany). For DNA extraction from agarose gels, the QIAEX II or QIAquick Gel Extraction Kits (Qiagen) were used.

\subsubsection{Enzymes}

The enzymes used in this study (see Table 2.2) were purchased from Fermentas (St. LeonRot, Germany), New England Biolabs (NEB, Frankfurt, Germany), and Bioline (Luckenwalde, Germany). The enzymes and the supplied buffers were used according to the manufacturers' instructions.

\subsubsection{High-quality plasmid preparation}

Plasmid DNA was isolated from E. coli overnight cultures by alkaline lysis (Birnboim and Doly 1979) using the buffers P1, P2, and P3 purchased from Qiagen (Hilden, Germany), according to the manufacturer's protocol. The solution containing the plasmid 
Table 2.2: Enzymes used in this study for molecular cloning.

\begin{tabular}{lll}
\hline Enzyme & Application & Supplier \\
\hline Restriction Endonucleases & Restriction digest & Fermentas/NEB \\
T4 Polynucleotide Kinase & Phosphorylation of primers & Fermentas \\
T4 DNA Ligase & Ligation & Fermentas \\
Pfu DNA polymerase & Cloning & Fermentas \\
Mango Taq DNA polymerase & RT-PCR, genotyping & Bioline \\
\hline
\end{tabular}

DNA was vigorously mixed with an equal volume of phenol:chloroform:isoamyl alcohol (25:24:1) in order to denature the proteins. Phase separation was achieved by centrifugation. The upper, aqueous phase was vigorously mixed with an equal volume of chloroform to extract the phenol. Phases were again separated by centrifugation. The plasmid DNA in the upper phase was transferred to a new reaction tube and precipitated with 0.7 volumes of isopropanol. The DNA pellet was dissolved in $30 \mu \mathrm{l}$ EB (10mM Tris/HCl, $\mathrm{pH} 8.5$, Qiagen).

\subsubsection{Polymerase chain reaction $(\mathrm{PCR})$}

Amplification of DNA fragments for RT-PCR or genotyping was done using Taq polymerase (Saiki et al. 1988) and TNK reaction buffer (Blanchard et al. 1993) in a $20 \mu \mathrm{l}$ or $25 \mu \mathrm{l}$ reaction. PCR for clonings was done using high-fidelity Pfu polymerase (Lundberg et al. 1991) together with the supplied buffer in a $50 \mu \mathrm{l}$ reaction volume. A typical $20 \mu \mathrm{l}$ reaction contains the following:

$\begin{array}{ll}2 \mu \mathrm{l} & \text { 10x reaction buffer } \\ 1 \mu \mathrm{l} & \text { template DNA (e.g. 20ng plasmid DNA) } \\ 0.5 \mu \mathrm{l} & \text { Forward primer }(50 \mu \mathrm{M}) \\ 0.5 \mu \mathrm{l} & \text { Reverse primer }(50 \mu \mathrm{M}) \\ 2 \mu \mathrm{l} & \text { dNTPs }(2.5 \mathrm{mM} \text { each }) \\ 0.2 / 1 \mu \mathrm{l} & \text { Taq/Pfu polymerase }\end{array}$

The PCR program used was adapted to the size of the amplicon and the melting temperature of the primers used.

10x TNK: 100mM Tris/HCl (pH 8.5), 15mM MgCl $2,500 \mathrm{mM} \mathrm{KCl,} \mathrm{50mM} \mathrm{NH} \mathrm{m}_{4} \mathrm{Cl}$. 


\subsubsection{DNA constructs}

All expression constructs are based on the lentiviral transfer vector FUGW (Lois et al. 2002), and its variant FHUG+W, which additionally contains an RNAi expression cassette (Schlüter et al. 2006) .

\subsubsection{PSD-93 knockdown constructs}

For the generation of RNAi constructs, 200pmole of each primer was phosphorylated by T4 PNK in a common reaction according to the manufacturers' instructions. The RNAi sequence was introduced into the plasmid pSuper or pBU6 by PCR using Pfu polymerase in the supplied buffer supplemented with DMSO (final concentration 5\%). The template DNA was digested with DpnI. The PCR product was purified and ligated for 2 hours at room temperature. $1 \mu$ l of the ligation reaction was electroporated into E. coli. Positive clones (identified by restriction digest and sequencing) were subcloned into FHUG+W using the EcoRI and ClaI/BstBI restriction sites.

The constructs rn931 and rn932 were kindly provided by Dr. Roger Nicoll (UCSF, CA, USA), and the mouse U6 promoter - RNAi expression cassette was subcloned into FHUG + W.

\subsubsection{PSD-93 overexpression}

The rat PSD-93 $\alpha_{2}$ coding sequence was a kind gift of Dr. Robert J. Wenthold. Mouse sequences of PSD-93 $\alpha_{1}$, PSD-93 $\beta$, PSD-93 $\delta$, and PSD-93 $\epsilon$ were generously provided by Dr. Guoping Feng, Duke University, USA (Parker et al. 2004). The EST clone AW060584 containing the N-terminus of PSD-93 $\gamma_{1 / 2}$ was purchased from Open Biosystems/Thermo Fisher Scientific (Bonn, Germany).

GFP-fusion constructs PSD-93 $\epsilon$ was obtained as a GFP-fusion protein with the linker sequence ADPPVAT. However, because it contained point mutations in the Cterminus of PSD-93, the C-terminus was substituted by the rat C-terminus (Wenthold) from the SacI to the C-terminal HindIII site. PSD-93є::GFP was then subcloned into FUGW downstream of the human ubiquitin $\mathrm{C}$ promoter to replace GFP. This DNA 


\section{MATERIALS AND METHODS}

construct was termed FUp93є GW. The N-termini of the individual isoforms were subcloned into FUp93 $\epsilon \mathrm{GW}$ to replace the $\epsilon$-N-terminus using the NheI site for PSD-93 $\alpha_{1}$, $\alpha_{2}$, and $\delta$, and the BstBI site for $\beta$.

The N-termini of PSD-93 $\gamma_{1}$ and PSD-93 $\gamma_{2}$ were amplified out of the EST clone AW060584 using the primer pair c93 $\gamma_{1} / \mathrm{c} 93 \gamma^{\prime}$ and $\mathrm{c} 93 \gamma_{2} / \mathrm{c} 93 \gamma^{\prime}$, respectively. The forward primers $\mathrm{c} 93 \gamma_{1}$ and $\mathrm{c} 93 \gamma_{2}$ introduced an EcoRI restriction site as well as a Kozak consensus sequence GCCACC 5' of the start codon (Kozak 1987). Using the restriction sites EcoRI and HindIII, the PCR products were subcloned into pBluescriptII SK(-) containing the C-terminus of PSD-93 $\alpha_{2}$. The N-termini were then cloned into FUp93 $\epsilon \mathrm{GW}$ to replace the $\epsilon$-N-terminus using EcoRI/NheI for the $\gamma_{1^{-}}$and EcoRI/BstBI for $\gamma_{2}$-isoform.

Additionally to the mouse PSD-93 $\alpha_{2}$ construct, PSD- $93 \alpha_{2}$ was cloned as a fulllength rat sequence and hence termed FUp93 $\alpha_{2} \mathrm{rGW}$. The coding sequence was amplified with the primers $93 \alpha_{2} 5 \mathrm{EcoRI}$ and $93 \alpha_{2} 3 \mathrm{BamHI}$ ' containing a 5' EcoRI site and a 3' BamHI site. The PCR product was cloned into FUp93 $\alpha_{2} \mathrm{GW}$ cut with the same enzymes. This rat construct was used for all subsequent experiments.

Bicistronic constructs Since the GFP tag might impede the protein's proper folding, bicistronic constructs were generated using the cytomegalovirus (CMV) internal ribosomal entry site (IRES). CMV-IRES-GFP was cut out of the plasmid pMES (Swartz et al. 2001) to replace GFP in FUGW. Rat PSD-93 $\alpha_{2}$ was inserted upstream of the CMV-IRES cassette and downstream of the human ubiquitin $\mathrm{C}$ promoter. The DNA construct was termed FUp93 $\alpha_{2}$ viGW. Subsequently, the N-terminus was swapped with the N-termini of the remaining PSD-93 isoforms to generate bicistronic constructs for all isoforms. Note that apart from the N-terminus, these constructs are based on the rat PSD-93 sequence.

\subsubsection{Replacement of PSD-95 with PSD-93 isoforms}

Knockdown of PSD-95 is efficiently achieved using the viral vector FH95pUGW that contains the RNAi construct sh95p (Schlüter et al. 2006). In order to knockdown PSD95 and replace it with PSD-93 isoforms, FH95pUGW was cut with PacI and BsrGI, and the backbone was retrieved. FUp93xGW $\left(\mathrm{x}=\alpha_{1 / 2}, \beta, \gamma_{1 / 2}, \delta, \epsilon\right)$ was cut with the same enzymes to retrieve the expression cassette for the PSD-93-GFP fusion proteins 
and ligated into the FH95pUGW backbone to yield FH95pUp93xGW ( $\mathrm{x}=\alpha_{1 / 2}, \beta, \gamma_{1 / 2}$, $\delta, \epsilon)$.

\subsubsection{Chimeric constructs}

PSD-93 exon 3 To clone the constructs with and without exon 3, I took advantage of the fact that there is a ClaI site in exon 2 of PSD-93. Thus, the N-termini of PSD-93 $\alpha_{2}$ and PSD-93 $\beta$ could be exchanged using this restriction site and keeping either the $\beta$-C-terminus (lacking exon 3 ) or the $\alpha_{2}$-C-terminus (containing exon 3 ).

PSD-93/PSD-95 chimeric constructs The chimeric constructs were cloned using the overexpression construct of PSD-95, FUp95GWEsm (Schlüter et al. 2006). Generating the constructs $95 \mathrm{~Np} 93$ and $93 \mathrm{~Np} 95$ was done in several steps. First, the PSD-95 N-terminus was subcloned from FUp95GWEsm into pBluescriptII SK(-) using the restriction sites EcoRI and SmaI (pB95). A circular PCR was performed on this construct to introduce Esp3I restriction sites using the primers cB95Esp and cB95Esp'. This temporary construct was termed pB95E. In parallel, PSD-93 $\alpha_{2}$ was subcloned from FUp93 $\alpha_{2}$ rGW into pBluescriptII SK(-) using the restriction sites EcoRI and BamHI/BglII (pB93). Then, a circular PCR was performed on this construct to introduce Esp3I restriction sites using the primers cB93Esp and cB93Esp'. This temporary construct was termed pB93E. pB95E was opened with EcoRI and Esp3I and the Nterminus of PSD-93 $\alpha_{2}$ (cut with the same enzymes out of pB93E) was pasted into this construct. This chimera was then cloned into FUp95GWEsm using the restriction enzymes EcoRI and NdeI to yield FUa2Np95GW (short: 93Np95). p93E was opened with EcoRI and Esp3I and the N-terminus of PSD-95 (cut with the same enzymes out of pB95E) was pasted into this construct. This chimera was then cloned into FUp93 $\alpha_{2}$ rGW using the restriction sites EcoRI and BstBI to yield FU95Np93a2rGW (short: 95Np93).

In order to replace the first 20 amino acids of PSD-93 $\alpha_{2}$ with the first nine amino acids of PSD-95, a circular PCR was performed on pB93 using the primers 95N1-9 and 95N1-9'. The PCR product was digested with Esp3I, ligated, and subcloned into FUp93 $\alpha_{2}$ rGW using the restriction sites EcoRI and BstBI to yield FU951-9p $93 \alpha_{2}$ rGW (short: 951-9p93). 


\section{MATERIALS AND METHODS}

In order to replace the first nine amino acids of PSD-95 with the first 20 amino acids of PSD-93 $\alpha_{2}$, a nested circular PCR was performed on pB95. In the first round, the primers $\mathrm{p} 93 \mathrm{~N}_{1-20} 1$ and $\mathrm{p} 93 \mathrm{~N}_{1-20} 1$ ' were used and the PCR product was gel extracted. For the second round, the primers $\mathrm{p} 93 \mathrm{~N}_{1-20} 2$ and $\mathrm{p} 93 \mathrm{~N}_{1-20} 2$ ' were used to introduce the remaining amino acids and an Esp3I site. The PCR product was digested with Esp3I, ligated and subcloned into FUp95GWEsm with EcoRI and NdeI to yield FU931-20p95GW (short: 931-20p95).

\subsubsection{Semi-quantitative RT-PCR}

\subsubsection{RNA isolation from tissue}

The RNA extraction from tissue using a chaotropic denaturing solution was initially described by Chomczynski and Sacchi (1987). The protocol using TRIzol (Invitrogen, Darmstadt, Germany) is an adaptation and was performed with minor modifications:

Mice were sacrificed by cervical dislocation, the desired tissue was dissected and immediately shock-frozen in liquid nitrogen. TRIzOL (50-100mg tissue/1ml TRIzoL) was added, and the tissue was homogenized by vortexing in the presence of glass beads. Extracellular membranes, polysaccharides and high molecular weight DNA as well as the glass beads were pelleted by centrifugation at $12,000 \mathrm{xg}$ for $10 \mathrm{~min}$ at $4^{\circ} \mathrm{C}$. The supernatant was incubated for $5 \mathrm{~min}$ at room temperature to permit complete dissociation of nucleoprotein complexes. 0.2 volumes of chloroform were added, the reaction tube was shaken vigorously by hand for $15 \mathrm{sec}$ and again incubated at room temperature for 2-3min. In order to dissociate the mixture into the lower red, phenol-chloroform containing, an interphase and a colorless upper aqueous phase which contains the RNA, the sample was centrifuged at $12,000 \mathrm{xg}$ for $15 \mathrm{~min}$ at $4^{\circ} \mathrm{C}$. The aqueous phase was transferred into a fresh reaction tube and the RNA was precipitated with 0.5 volumes of isopropanol. The samples were incubated at room temperature for $10 \mathrm{~min}$ and centrifuged at $12,000 \mathrm{xg}$ for $10 \mathrm{~min}$ at $4^{\circ} \mathrm{C}$. The RNA pellet was washed once with $75 \%$ ethanol and air-dried for 5-10min. $20 \mu \mathrm{l}$ deionized $\mathrm{H}_{2} \mathrm{O}$ was added and the RNA was allowed to dissolve by incubating for $10 \mathrm{~min}$ at $55^{\circ} \mathrm{C}$. The concentration was determined by measuring the absorption at $260 \mathrm{~nm}$ with a photospectrometer (concentration of $40 \mu \mathrm{g} / \mathrm{ml}$ is equal to an absorption of 1 ). The RNA was stored at $-80^{\circ} \mathrm{C}$. 


\subsubsection{Reverse Transcription}

The extracted total RNA includes mRNA. This mRNA was transcribed into cDNA using random primers and reverse transcriptase, also known as RNA-dependent DNA polymerase. 500ng total RNA were reverse transcribed according to the manufacturer's protocol (High Capacity cDNA Transcription Kit, Applied Biosystems/Life Technologies, Carlsbad, CA, USA).

\subsubsection{Polymerase chain reaction on cDNA}

The PCR on cDNA is essentially done as described in Section 2.1.7. $1 \mu$ l of cDNA template was used for a $25 \mu \mathrm{l}$ reaction. The cycling conditions were $2 \mathrm{~min} 94^{\circ} \mathrm{C}, 30 \mathrm{x}$ (30sec $94^{\circ} \mathrm{C}, 30 \sec 55^{\circ} \mathrm{C}, 1.5 \min 72^{\circ} \mathrm{C}$ ), $5 \min 72^{\circ} \mathrm{C}$.

For the N-terminus, the following primer pairs were used:

$\begin{array}{ccc}\text { Isoform } & \text { Primer pair } & \text { Expected size } \\ \alpha_{1} & 16484,16492 & 1000 \mathrm{bp} \\ \alpha_{2} & 16485,16492 & 1000 \mathrm{bp} \\ \beta & 17984,12078 & 500 \mathrm{bp} \\ \gamma_{1 / 2} & 16490,16491 & 1000 \mathrm{bp} \\ \delta & 16486,16492 & 1000 \mathrm{bp} \\ \epsilon & 16487,18577 & 800 \mathrm{bp}\end{array}$

The PCR products were separated on an agarose gel and visualized by ethidium bromide under UV light. The gel pictures were digitalized, and the average pixel intensity was determined using the program ImageJ. For the bar graph (Fig. 3.3B, p. 41), the intensities were normalized by division by the sum of the intensities of one isoform.

For the C-terminus, the primers E15, E16, E17a, E17, E17a', E17', E17b', and E18' were used in the combinations shown in Figure 3.4B1, B2 (p. 42). The cycling conditions were $2 \min 94^{\circ} \mathrm{C}, 30 \mathrm{x}\left(30 \sec 94^{\circ} \mathrm{C}, 30 \sec 55^{\circ} \mathrm{C}, 30 \sec 72^{\circ} \mathrm{C}\right), 5 \min 72^{\circ} \mathrm{C}$.

\subsection{Cell culture techniques}

All tissue culture plates were obtained from Greiner Bio-One (Frickenhausen, Germany), and filters and membranes from Millipore (Schwalbach, Germany). Cell culture grade chemicals were purchased from Roth (Karlsruhe, Germany), Merck (Darmstadt, Germany), and Sigma-Aldrich (München, Germany). Medium and supplements 


\section{MATERIALS AND METHODS}

were obtained from Biochrom (Berlin, Germany), Invitrogen/Life Technologies (Darmstadt, Germany), HyClone/Thermo Fisher Scientific (Waltham, MA, USA), Roche (Mannheim, Germany), and Mirus Bio (Madison, WI, USA).

\subsubsection{HEK293T cell culture}

HEK293 cells are a cell line of human embryonic kidney cells which have been immortalized (Graham et al. 1977). The HEK293T strain holds the SV40 large T-antigen allowing the replication of plasmids containing the SV40 origin of replication, and thus enable enhanced expression of specific gene products.

HEK293T cells were maintained in a $37^{\circ} \mathrm{C}$ humified incubator with $5 \% \mathrm{CO}_{2}$ in HEK medium on $10 \mathrm{~cm}$ cell culture dishes. The cells were passaged 1:5-1:20 every two to three days using standard procedures of trypsin-mediated dissociation of confluent monolayer cultures (Masters and Stacey 2007).

HEK medium: High-glucose Dulbecco's Modified Eagle's Medium (Biochrom), 2mM Glutamax (Invitrogen), 10\% fetal calf serum (Biochrom).

\subsubsection{Transfection of HEK293T cells}

HEK293T cells in a 24 well plate were transfected with 250-500ng of plasmid DNA when they were 60-70\% confluent. For the transfection Fugene6 (Roche) or TransIt (Mirus Bio) transfection reagents was used, according to the manufacturers' protocols. After 24 hours the cells were harvested as described in Section 2.4.2.

\subsubsection{Lentivirus production}

Lentiviruses are a class of retroviruses equipped with the ability to infect both dividing and non-dividing cells, and are thus capable of transducing neurons (Lewis et al. 1992). The viral transfer vectors used for the generation of lentiviral particles are described in detail in Section 2.1.8.

For the production of lentiviral vectors, the transfer vector, the HIV-1 packaging vector $\Delta 8.9$, and the vsvg envelope glycoprotein vector were cotransfected into $60-70 \%$ confluent HEK293T cells using Fugene6 (Roche) or TransIt (Mirus Bio) transfection reagents. Small scale virus production was performed in 6 well tissue plates using $2 \mu \mathrm{g}$ of transfer vector, $1.5 \mu \mathrm{g}$ of $\Delta 8.9$ and $0.5 \mu \mathrm{g}$ of vsvg. For large scale virus preparation, 
T-75 culture flasks were used with $10 \mu \mathrm{g}$ of transfer vector, $7.5 \mu \mathrm{g}$ of $\Delta 8.9$ and $2.5 \mu \mathrm{g}$ of vsvg. 24 hours after the transfection, the HEK293T culture dish was moved to a $32^{\circ} \mathrm{C}$ humified incubator with $5 \% \mathrm{CO}_{2} .36-48$ hours after the transfection, the medium was collected, and centrifuged for 5min at 800xg to remove HEK293T cell debris. In case of the small scale virus production, the supernatant was aliquoted and stored at $-80^{\circ} \mathrm{C}$. In case of the large scale virus production, the supernatant was filtered through a $0.45 \mu \mathrm{m}$ PVDF membrane (Millipore), and then centrifuged at 36,500xg for 90min to concentrate the viral particles. The pellet was allowed to dissolve in $100 \mu \mathrm{l} \mathrm{VSB}$ at $4^{\circ} \mathrm{C}$ overnight. The virus was aliquoted and stored at $-80^{\circ} \mathrm{C}$.

VSB: 20mM Tris-HCl (pH 8.0), 250mM NaCl, $5 \%$ Sorbitol.

\subsubsection{Dissociated hippocampal cultures}

Dissociated hippocampal cultures were prepared from newborn (P0) or one day old (P1) mice or Wistar rats of both sexes. The hippocampi were dissected, the dentate gyri as well as the fimbriae carefully removed, and the hippocampi were digested for 30min at $37^{\circ} \mathrm{C}$ with papain, according to the method of Huettner and Baughman (1986). Following trituration with a fire polished glass pipette, 100,000 cells were plated per well of a poly-D-lysine-coated 24 well plate into culture medium. Astrocyte growth was inhibited with FUDR (Sigma-Aldrich) at DIV4. On DIV7, 50\% of the medium was exchanged against culture medium that has been conditioned by astrocytes for $24 \mathrm{~h}$. The feeding was repeated every 3-7 days, dependent on the density of the culture.

Culture medium: DMEM/HamsF12 (Biochrom) supplemented with 2\% B27 (Invitrogen) and 1\% Glutamax (Invitrogen).

\subsubsection{Infection of dissociated cultures}

On DIV7, after the medium was exchanged, cultures were infected with $30-100 \mu$ l virus supernatant.

\subsubsection{Organotypic hippocampal slice cultures}

Organotypic hippocampal slices were prepared from postnatal day 7-9 Wistar rats of both sexes, according to the method of Stoppini et al. (1991) with minor modifications. Animals were anaesthetized with Isoflurane. After decapitation, the hippocampi of 


\section{MATERIALS AND METHODS}

both hemispheres were dissected in ice-cold cutting buffer and fimbriae were removed. The hippocampi were placed on a guillotine base and $300 \mu \mathrm{m}$ thick slices were cut with a wire frame. The slices were collected with a plastic Pasteur pipette and transferred into artificial cerebrospinal fluid (ACSF) for recovery. Slices were washed in BME and then plated onto rectangular membrane pieces on porous membrane discs $(0.4 \mu \mathrm{m}$, Millipore) in culture dishes containing $20 \% \mathrm{HK}$. The slices were incubated at $37^{\circ} \mathrm{C}$ in a $5 \% \mathrm{CO}_{2}$ environment. On DIV1, the slices were transferred to $34^{\circ} \mathrm{C}$, and the culture medium was replaced with fresh $20 \%$ HK. On DIV4 or DIV5 the culture medium was replaced by $5 \% \mathrm{HK}$, and renewed every other day.

20\% HK: 49\% BME (Biochrom), 25\% EBSS, 25mM HEPES (Biochrom), 20\% Donor Equine Serum (heat-inactivated for $30 \mathrm{~min}$ at $55^{\circ} \mathrm{C}$, HyClone), $28 \mathrm{mM}$ D-Glucose, $1 \mathrm{mM}$ Glutamax (Invitrogen), $88 \mu \mathrm{g} / \mathrm{ml}$ L-Ascorbic acid, $1 \mu \mathrm{g} / \mathrm{ml}$ Insulin, $0.25 \% 100 \mathrm{X}$ MEM-Vitamins, 0.49\% 50X MEM-amino acids.

5\% HK: 63.3\% BME (Biochrom), 25\% EBSS, 25mM HEPES (Biochrom), 5\% Donor Equine Serum (heat-inactivated for $30 \mathrm{~min}$ at $55^{\circ} \mathrm{C}, \mathrm{HyClone}$ ), $28 \mathrm{mM}$ D-Glucose, $2 \mathrm{mM}$ Glutamax (Invitrogen), $88 \mu \mathrm{g} / \mathrm{ml}$ L-Ascorbic acid, $1 \mu \mathrm{g} / \mathrm{ml}$ Insulin, 0.32\% 100X MEM-Vitamins, 0.63\% 50X MEM-amino acids.

EBSS: $1.8 \mathrm{mM} \mathrm{CaCl}_{2} * 2 \mathrm{H}_{2} \mathrm{O}, 1 \mathrm{mM} \mathrm{NaH}_{2} \mathrm{PO}_{4} * \mathrm{H}_{2} \mathrm{O}, 0.8 \mathrm{mM} \mathrm{MgSO}_{4} * 7 \mathrm{H}_{2} \mathrm{O}, 116 \mathrm{mM}$

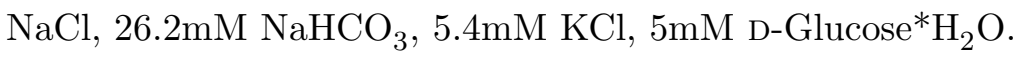

Sucrose Cutting Buffer: 204mM Sucrose, 26mM $\mathrm{NaHCO}_{3}$, 10mM D-Glucose, $2.5 \mathrm{mM} \mathrm{KCl}, 1 \mathrm{mM} \mathrm{NaH} \mathrm{PO}_{4} * \mathrm{H}_{2} \mathrm{O}, 4 \mathrm{mM} \mathrm{MgSO}_{4} * 7 \mathrm{H}_{2} \mathrm{O}, 1 \mathrm{mM} \mathrm{CaCl} \mathrm{Cl}_{2} * 2 \mathrm{H}_{2} \mathrm{O}, 4 \mathrm{mM} \mathrm{L}-$ Ascorbic acid; sterile filtered.

ACSF (recovery): $119 \mathrm{mM} \mathrm{NaCl}, 26 \mathrm{mM} \mathrm{NaHCO} 3,20 \mathrm{mM}$ D-Glucose, $2.5 \mathrm{mM}$ $\mathrm{KCl}, 1 \mathrm{mM} \mathrm{NaH} \mathrm{PO}_{4}, 4 \mathrm{mM} \mathrm{MgSO}{ }_{4} * 7 \mathrm{H}_{2} \mathrm{O}, 4 \mathrm{mM} \mathrm{CaCl}{ }_{2} * 2 \mathrm{H}_{2} \mathrm{O}$. Sterile filtered and oxygenated for $30 \mathrm{~min}$ with $95 \% \mathrm{O}_{2} / 5 \% \mathrm{CO}_{2}$ before use.

\subsubsection{Virus injection into slice cultures}

At DIV1 or DIV2, concentrated viral particles were injected into two to three sites of the CA1 pyramidal cell layer using the Nanoject II device (Drummond Scientific, Broomall, PA, USA) with the supplied capillary tips, and mounted on a custom-built micromanipulator. The amount of virus injected was in the low $\mathrm{nl}$ range, dependent on 
the virus titer, and was adjusted such that single cells in CA1 got transduced. After the injection, the culture medium was exchanged by fresh $20 \%$ HK.

\section{$2.3 \quad$ PSD-93 knock-out strain and genotyping}

The PSD-93 KO mouse line is described in McGee et al. (2001), and was kept in the institute's animal facility. The genotype of the animals was determined by PCR according to the protocol described by The Jackson Laboratory (Bar Harbor, ME, USA): $2 \mathrm{~mm}$ mouse tail was digested overnight in $200 \mu \mathrm{l}$ PBND at $55^{\circ} \mathrm{C}$. Samples were boiled for $10 \mathrm{~min}$, and $2 \mu \mathrm{l}$ was used for multiplex PCR genotyping in a $20 \mu \mathrm{l}$ reaction using the primers p93-intron, p93-exon', and p93-neo', and Taq polymerase. The PCR products were analyzed by gel electrophoresis to show the presence of the wildtype allele (330bp product) and/or the knockout allele (750bp product).

PBND: $10 \mathrm{mM}$ Tris/ $\mathrm{HCl}$ ( $\mathrm{pH} 8.3$ ), $50 \mathrm{mM} \mathrm{KCl}, 2.5 \mathrm{mM} \mathrm{MgCl}_{2}, 0.01 \%$ gelatin, $0.45 \%$ Nonidet P-40, 0.45\% Tween $20,100 \mu \mathrm{g} / \mathrm{ml}$ proteinase K.

\subsection{Protein-Biochemistry}

\subsubsection{Antibodies}

Antibodies were obtained from Invitrogen (Darmstadt, Germany), UC Davis/NIH NeuroMab Facility (Davis, CA, USA), Assay Designs (Ann Arbor, MI, USA), Santa Cruz Biotechnology, Inc. (Heidelberg, Germany), Li-COR Biosciences (Bad Homburg, Germany), and Millipore (Molsheim, France). The polyclonal PSD-93 antiserum (rabbit) was a kind gift of Dr. David Bredt (Eli Lilly, Indianapolis, IN, USA). The PSD-93 $\delta$ antiserum raised against the N-terminal amino acids 1-20 (MNAYLTKQHSCSRGSDGMDI) of PSD-93 $\delta$ was generously provided by Dr. Mark Leyland, University of Leicester, UK (Leyland and Dart 2004). The Nedd4-1 polyclonal antibody was a kind gift of Prof. Nils Brose and Dr. Hiroshi Kawabe, Max Planck Institute of Experimental Medicine, Göttingen, Germany (Kawabe et al. 2010).

\subsubsection{Sample preparation: HEK293T cells}

HEK293T cells were washed once with $1 \mathrm{ml}$ PBS without $\mathrm{Ca}^{2+} / \mathrm{Mg}^{2+}$ (Biochrom), on ice. The cells were lysed with $100 \mu$ l RIPA buffer in the well, and the lysate was 
Table 2.3: Antibodies used in this study for western blotting.

\begin{tabular}{|c|c|c|}
\hline Antibody (host) & Dilution & Supplier/Reference \\
\hline \multicolumn{3}{|l|}{ Primary antibodies } \\
\hline$\alpha$-GFP (rabbit) & $1: 5000$ & Invitrogen \\
\hline$\alpha$-GFP (mouse) & $1: 2000$ & NeuroMab \\
\hline$\alpha$-GluR2 (mouse) & $1: 2000$ & NeuroMab \\
\hline$\alpha$-NR2A (rabbit) & $1: 2000$ & Upstate/Millipore \\
\hline$\alpha$-NR2B (mouse) & $1: 2000$ & NeuroMab \\
\hline$\alpha$-PSD-95 (mouse) & $1: 5000$ & NeuroMab \\
\hline$\alpha$-PSD-93 N18/30 (mouse) & $1: 2000$ & NeuroMab \\
\hline$\alpha$-PSD-93 (rabbit) & $1: 2000$ & Dr. David Bredt \\
\hline$\alpha$-SAP97 (mouse) & $1: 2000$ & Assay Designs \\
\hline$\alpha$-SAP97 (mouse) & $1: 2000$ & NeuroMab \\
\hline$\alpha$-SAP102 (mouse) & $1: 2000$ & NeuroMab \\
\hline$\alpha$-mortalin (mouse) & $1: 10000$ & NeuroMab \\
\hline$\alpha$-GAPDH (mouse) & $1: 10000$ & Chemicon/Millipore \\
\hline$\alpha-14-3-3 \beta$ (mouse) & $1: 1000$ & Santa Cruz \\
\hline$\alpha$-Nedd4-1 (rabbit) & $1: 2500$ & Kawabe et al. (2010) \\
\hline$\alpha$-PSD-93 $\delta$ (serum, rabbit) & $1: 250$ & Leyland and Dart (2004) \\
\hline \multicolumn{3}{|l|}{ Secondary antibodies } \\
\hline$\alpha$-mouse Alexa Fluor 680 (goat) & 1:15000 & Invitrogen \\
\hline$\alpha$-rabbit Alexa Fluor 680 (goat) & $1: 15000$ & Invitrogen \\
\hline$\alpha$-mouse IR800 (goat) & $1: 15000$ & Li-COR Biosciences \\
\hline$\alpha$-rabbit IR800 (goat) & $1: 15000$ & Li-COR Biosciences \\
\hline
\end{tabular}


transferred to a reaction tube. Insoluble material was pelleted by centrifugation (30min, $20,000 \mathrm{xg}$ ). $50 \mu \mathrm{l}$ of $4 \mathrm{x}$ sample buffer was added to the supernatant, and the sample was boiled for 10min before subjecting 10-15 $\mu$ l of the sample to SDS-PAGE.

RIPA buffer: $50 \mathrm{mM} \mathrm{NaH}_{2} \mathrm{PO}_{4}(\mathrm{pH} 7.4), 150 \mathrm{mM} \mathrm{NaCl}, 1 \%$ Triton-X100, $0.1 \%$ SDS, $0.5 \%$ deoxycholic acid, 2mM EDTA, 1x Complete Proteinase inhibitor cocktail (Roche).

4x Sample buffer: 0.8M Tris/HCl ( $\mathrm{pH} 8.4$ ), $40 \%$ (w/v) glycerol, $8 \%$ SDS, $2 \mathrm{mM}$ EDTA, $0.075 \%$ bromphenolblue. $13 \mathrm{mM}$ dithiothreitol was added before use.

\subsubsection{Sample preparation: dissociated cultures}

Dissociated cultures were washed once with $1 \mathrm{ml}$ PBS containing $\mathrm{Ca}^{2+} / \mathrm{Mg}^{2+}$ (Biochrom), on ice. The cells were then harvested using $30 \mu \mathrm{l} 2 \mathrm{x}$ sample buffer (p. 27) per well of a 24 well plate, and the lysate was transferred into a reaction tube. The sample was boiled for 10min before subjecting 10-15 $\mu$ l of the sample to SDS-PAGE.

\subsubsection{Sample preparation: tissue}

Wildtype (WT) or PSD-93 KO mice were sacrificed, the brain immerged in cold homogenization buffer (HB), and the hippocampi of both hemispheres were dissected on a cold surface. Two different protocols were used:

A) The hippocampi were homogenized in HB by sonication. The nuclear fraction was pelleted (10min, $800 \mathrm{xg}$ ), and the supernatant $\mathrm{S} 1$ extracted for 1 hour by the addition of sodium N-lauryl sarcosine to a final concentration of $1 \%$. Insoluble proteins were pelleted by centrifugation (1h, 100,000xg).

B) Alternatively, the hippocampi were homogenized in HB with a glass/teflon potter (10 strokes) yielding the homogenate $\mathrm{H}$. The nuclear fraction was pelleted (10min, $800 \mathrm{xg}$ ), and the supernatant S1 centrifuged (15min, 9200xg) to enrich in synaptosomes (in the pellet $\mathrm{P} 2$ ).

The protein concentration of the lysate was determined by BCA assay, adjusted and an equal volume of $2 \mathrm{x}$ sample buffer (p. 27) was added. The samples were subjected to SDS-PAGE, or stored at $-80^{\circ} \mathrm{C}$.

HB: 320mM Sucrose, 2mM EDTA (pH 7.4), 1x Complete Proteinase inhibitor cocktail (Roche). 


\section{MATERIALS AND METHODS}

\subsubsection{Protein concentration with BCA assay}

The protein concentration was determined using the bicinchoninic acid (BCA) assay (Smith et al. 1985) and according to the instruction of the BCA Protein Assay Kit (Pierce/Thermo Fisher Scientific, Bonn, Germany).

\subsubsection{SDS-PAGE and western blot}

Protein samples were separated according to their molecular weight by SDS-Polyacrylamid gel electrophoresis (SDS-PAGE, introduced by Shapiro et al. 1967) in a 4-20\% precast NuPage 4-12\% BisTris gel (Invitrogen) for up to 5 hours at $120 \mathrm{~V}$, dependent on the desired resolution and molecular weight of the protein of interest.

Following SDS-PAGE, the proteins were western blotted (Towbin et al. 1979): The proteins were transferred to a $0.2 \mu \mathrm{m}$ nitrocellulose membrane (Whatman/GE Healthcare, Maidstone, UK) for two hours at 200mA, or overnight at $40 \mathrm{~mA}$. Successful transfer was confirmed by incubation with Ponceau S dye (Sigma-Aldrich). The membrane was blocked in blocking solution for one hour at room temperature. Afterwards, the membrane was incubated with primary antibodies in TBS-T-2.5 at dilutions shown in Table 2.3, overnight at $4^{\circ} \mathrm{C}$. The membrane was washed three times for $10 \mathrm{~min}$ with TBS-T and then incubated for 1 hour at room temperature with the appropriate secondary antibody diluted in TBS-T. Again, the membrane was washed three times for 10min with TBS-T, rinsed with TBS, and stored at $4^{\circ} \mathrm{C}$ in TBS until analysis.

The membrane was imaged using an Odyssey Infrared Imaging System (Li-COR Biosciences, Bad Homburg, Germany). The scan resolution was set to $160 \mu \mathrm{m}$. The quantification was performed with the Odyssey analysis software using integrated intensity with median background correction.

For mass spectrometry analysis, the proteins were not transferred to nitrocellulose membranes but were instead stained with Coomassie blue. For this, the gel was incubated in Coomassie staining solution for $15 \mathrm{~min}$ at $50^{\circ} \mathrm{C}$ under agitation. The gel was boiled in excess $\mathrm{H}_{2} \mathrm{O}$ for $10 \mathrm{~min}$, and then incubated in Coomassie destaining solution. The destaining solution was regularly exchanged until the background was destained. 
20x SDS running buffer: 1M MOPS, 1M Tris, 20mM EDTA, 2\% SDS.

Transfer buffer: 3g/l Tris, 14.4g/l Glycin, 20\% Methanol.

TBS: 20mM Tris/HCL (pH 7.5), 140mM NaCl.

TBS-T: TBS, $0.1 \%$ Tween 20.

Blocking solution: TBS-T, $5 \%$ milk powder.

TBS-T-2.5: TBS-T, $2.5 \%$ milk powder.

Coomassie staining solution: $0.15 \%$ Coomassie Brilliant Blue R250, 10\% Acetic acid, $25 \%$ Isopropanol.

Coomassie destaining solution: 30\% Methanol, $10 \%$ Acetic acid.

\subsubsection{Immunoprecipitation (IP)}

All steps were performed at $4{ }^{\circ} \mathrm{C}$ or on ice, unless otherwise indicated. The protein A/Gor anti-mouse-agarose-suspension was washed with TET before use. The following antibodies and agarose combinations were used for immunoprecipitation:

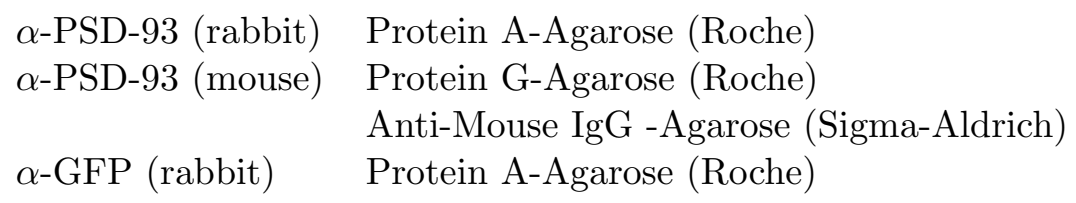

\subsubsection{IP from tissue}

In order to isolate PSD-93 from hippocampal tissue, the hippocampi were dissected and homogenized in HB (250 $\mu$ l/hippocampus, see p. 27) by sonication. Cellular debris was removed by centrifugation $(800 \mathrm{xg}, 10 \mathrm{~min})$. The supernatant was transferred to a new reaction tube, an equal volume of $2 \mathrm{x}$ TET was added, and the samples were agitated for $30 \mathrm{~min}$. Sodium N-lauryl sarcosine was added to a final concentration of $1 \%$, and proteins were extracted for $1 \mathrm{~h}$. Insoluble proteins were pelleted by centrifugation $(1 \mathrm{~h}$, 100,000xg). The lysate was transferred to a new reaction tube. To reduce background, the lysate was pre-cleared with protein A/G- or anti-mouse-agarose. For this, 30 $\mu$ l of agarose-suspension was added to the sample, and incubated for $2 \mathrm{~h}$ on a rocking platform or a rotator. The unspecifically bound proteins were pelleted with the agarose beads by centrifugation (1min, 2000xg). $5 \mu \mathrm{g}$ of antibody was added to the lysate and 


\section{MATERIALS AND METHODS}

incubated overnight, agitating. $50 \mu \mathrm{l}$ of protein $\mathrm{A} / \mathrm{G}$ - or anti-mouse-agarose was added to the sample and incubated for 2-4 hours, agitating. The antigen-antibody-agarose complexes were collected by centrifugation $(1 \mathrm{~min}, 2000 \mathrm{xg})$. The pellet was washed with $1 \mathrm{ml}$ TET for 20min, agitating. Again, the antigen-antibody-agarose complexes were collected by centrifugation, the supernatant discarded. The washing steps were repeated three times. After the last centrifugation, 30 $\mu \mathrm{l} 2 \mathrm{x}$ sample buffer (p. 27) was added to the beads and the samples were boiled for 10min to dissociate the antigen from the antibody-agarose complex. The supernatant was analyzed by SDS-PAGE.

\subsubsection{IP from dissociated cultures}

Dissociated neuronal cultures from 6 wells of a 24 -well plate were pooled and harvested with $500 \mu \mathrm{l}$ Buffer A. The cell suspension was incubated for 15min, agitating. The Triton-X100 insoluble material was collected by centrifugation $(32,000 x g, 20 \mathrm{~min})$ and extracted with $200 \mu$ l Buffer A supplemented with sodium N-lauryl sarcosine (final concentration of $1 \%$ ). The pellet was dissociated by pipetting and incubation on ice for $10 \mathrm{~min}$. $2 \mu \mathrm{g}$ of antibody was added to the sample and incubated overnight, agitating. The antigen-antibody complexes were captured by adding $30-50 \mu$ l of protein A/G- or anti-mouse-agarose for 2-4 hours. Following centrifugation (1min, 2000xg), the supernatant was discarded and the pellet washed with $1 \mathrm{ml}$ TET for 20min, agitating. Again, the antigen-antibody-agarose complexes were collected by centrifugation, the supernatant discarded. The washing steps were repeated three times. After the last centrifugation, $30 \mu \mathrm{l} 2 \mathrm{x}$ sample buffer (p. 27) was added to the agarose and the sample was boiled for 10min to dissociate the antigen from the antibody-agarose complex. The supernatant was analyzed by SDS-PAGE.

TET: $20 \mathrm{mM}$ Tris-HCl (pH 8 at $\left.4^{\circ} \mathrm{C}\right), 1 \mathrm{mM}$ EDTA (pH 8), 1.3\% Triton-X100.

2x TET: $40 \mathrm{mM}$ Tris-HCl (pH 8 at $\left.4^{\circ} \mathrm{C}\right), 2 \mathrm{mM}$ EDTA (pH 8), 2.6\% Triton-X100, 1x Complete Proteinase inhibitor cocktail (Roche).

Buffer A: 1mM EDTA (pH 7.4), 50mM NaF, $1 \mathrm{mM} \mathrm{NaVO}_{4}, 50 \mathrm{mM}^{\mathrm{m}}$ Hepes (pH 7.3), 0.25\% Triton-X100, 1x Complete Proteinase inhibitor cocktail (Roche). 


\subsection{Identification of PSD-93 isoforms by mass spectro- metry}

The analysis of PSD-93 isoforms by mass spectrometry was done in collaboration with Monika Raabe and Dr. Henning Urlaub (Bioanalytical Mass Spectroscopy Group, Max Planck Institute for Biophysical Chemistry, Göttingen, Germany).

The band corresponding to PSD-93 was cut from a 4-20\% precast NuPAGE 4-12\% Bis-Tris gel (Invitrogen) and the protein was digested in-gel with either trypsin or chymotrypsin (Rosenfeld et al. 1992; Shevchenko et al. 1996). After cleavage, peptides were extracted and analyzed with an LTQ Orbitrap XL (Thermo Fisher Scientific, Waltham, MA, USA).

The MS and MS/MS spectra were searched against the in-house refseq_mouse database using the Mascot algorithm (Perkins et al. 1999). Because the sequences of the individual isoforms of PSD-93 were not present in this database, fasta-files for all PSD-93 isoforms were incorporated into it. Mascot uses a probability-based approach. The output for a peptide or a protein is presented as a score $S$, which is given by $S=-10 * \log _{10}(P)$, where $P$ is the probability that an observed hit is a false positive which is produced by a random match. For the protein score, the threshold depends on the size of the database queried. The refseq_mouse database contained 35,959 protein entries. Thus, for a $5 \%$ chance to obtain a false positive match, $P=0.05 * 1 / 35959$, and the equivalent Mascot score is $S=59$. The ions score for an MS/MS match is calculated identically, but depends on the number of peptides that fall into the mass tolerance window about the precursor mass. Thus, the ions score cannot be calculated a priori. In general, an ions score equal to or greater than 20 is considered a good hit (personal communication with Monika Raabe and Carla Schmidt, Bioanalytical Mass Spectroscopy Group, Max Planck Institute for Biophysical Chemistry, Göttingen, Germany). For all N-terminal peptides of the PSD-93 isoforms, the MS/MS mass spectra were manually inspected to assure that the amino acid sequence is well resembled in the mass spectrum. 


\subsection{Electrophysiology}

\subsubsection{Equipment}

For the electrophysiological measurements, the following equipment was used:

\begin{tabular}{|c|c|}
\hline Instrument & Supplier \\
\hline Puller P-97 & Sutter Instrument (Novato, CA, USA) \\
\hline MultiClamp700B amplifier & Molecular Devices (Sunnyvale, CA, USA) \\
\hline ITC-18 data acquisition board & InstruTECH/HEKA (Lambrecht, Germany) \\
\hline Micromanipulator MP-225 & Sutter Instrument (Novato, CA, USA) \\
\hline IR-1000 Infrared CCD Camera & DAGE-MTI (Michigan City, IN, USA) \\
\hline Stimulator ISO-FLEX & A.M.P.I. (Jerusalem, Israel) \\
\hline $\begin{array}{l}\text { Borosilicate (KG-33) glass } \\
\text { (inner } \varnothing 1 \mathrm{~mm} \text {, outer } \varnothing 1.5 \mathrm{~mm})\end{array}$ & King Precision Glass, Inc. (Claremont, CA, USA) \\
\hline Double-barrel glass TST150-6 & World Precision Instruments (Sarasota, FL, USA) \\
\hline
\end{tabular}

\subsubsection{Data acquisition}

Recordings were performed 4-9 days after infection. A single slice was transferred into the recording chamber that was constantly perfused with oxygenated ACSF (2$3 \mathrm{ml} / \mathrm{min}$ ), kept at $\sim 28^{\circ} \mathrm{C}$. Patch pipettes (open pipette resistance $3-6 \mathrm{M} \Omega$ ) were filled with intracellular solution. Simultaneously, pairs of infected and control cell were voltage-clamped in whole-cell configuration. Responses were simultaneously evoked in both cells using a single bipolar stimulation electrode filled with ACSF. AMPAR EPSCs were recorded at $-60 \mathrm{mV}$. NMDAR EPSCs were measured at $+40 \mathrm{mV}$ and $60 \mathrm{msec}$ after the peak, a time point at which AMPAR-mediated currents are mostly absent. The responses were monitored online and analyzed online or offline using a custom-written acquisition and analysis software implemented in IGOR Pro. The signals were filtered at $4 \mathrm{kHz}$ and digitalized at $10 \mathrm{kHz}$ using an ITC-18 acquisition board. For each cell pair a minimum of 40 sweeps was collected and averaged. The stimulation strength was adjusted such that the control cell amplitude of AMPAR EPSCs was around 30$50 \mathrm{pA}$. A $5 \mathrm{mV}$ hyperpolarizing step was used to monitor the input resistance and series resistance over time.

ACSF: $119 \mathrm{mM} \mathrm{NaCl}, 26 \mathrm{mM} \mathrm{NaHCO} 3,20 \mathrm{mM}$ D-Glucose, $4 \mathrm{mM} \mathrm{MgSO}_{4} * 7 \mathrm{H}_{2} \mathrm{O}$, $2.5 \mathrm{mM} \mathrm{KCl}, 1 \mathrm{mM} \mathrm{NaH} \mathrm{PO}_{4}, 4 \mathrm{mM} \mathrm{CaCl}{ }_{2} * 2 \mathrm{H}_{2} \mathrm{O}$. The ACSF was oxygenated for 
30 min with $95 \% \mathrm{O}_{2} / 5 \% \mathrm{CO}_{2}$ before use. 1-5 $\mu \mathrm{M}$ 2-chloroadenosine was added to reduce polysynaptic activity, and $50 \mu \mathrm{M}$ picrotoxin to block inhibitory transmission and isolate EPSCs.

Intracellular solution: $117.5 \mathrm{mM} \mathrm{MeSO}_{3} \mathrm{H}, 10 \mathrm{mM} \mathrm{HEPES}, 17.75 \mathrm{mM} \mathrm{CsCl}, 10 \mathrm{mM}$ TEA-Cl, 0.25mM EGTA, 10mM D-Glucose, 2mM MgCl $2{ }_{2} * 6 \mathrm{H}_{2} \mathrm{O}, 4 \mathrm{mM} \mathrm{Na}_{2}$ ATP, $0.3 \mathrm{mM}$ NaGTP. CsOH $50 \%(\mathrm{w} / \mathrm{v}$ ) was used to adjust $\mathrm{pH}$ to 7.3, and the solution was cooled to $4^{\circ} \mathrm{C}$ before adding $\mathrm{Na}_{2}$ ATP and NaGTP. The osmolarity was adjusted to $290 \mathrm{mOsm}$ with $\mathrm{CsCl}$. The solution was filtered with a $0.2 \mu \mathrm{m}$ syringe filter, aliquoted and stored at $-80^{\circ} \mathrm{C}$ for up to 2 months.

\subsubsection{Pharmacology}

D-AP-V, NBQX, Bicuculline, and Picrotoxin were obtained from Ascent Scientific (Bristol, UK). GYKI53655 (LY303070) was kindly provided by Dr. Juan Lerma (Instituto de Neurociencias, Alicante, Spain). 2-chloroadenosine was purchased from BioLog (Bremen, Germany).

\subsubsection{Statistical analysis}

The statistical analysis of the electrophysiological data was done using non-parametric tests using the software R Project. First, the outliers defined as being outside the range $\left[Q_{1}-1.5 * I Q R ; Q_{3}+1.5 * I Q R\right], I Q R=Q_{3}-Q_{1}$, were eliminated. $Q_{1}$ and $Q_{3}$ denote the $25^{\text {th }}$ and $75^{\text {th }}$ percentiles, respectively. Comparison between control and infected cell responses were analyzed using the Wilcoxon Signed-Rank test statistic. Comparison between two different constructs was performed using the Wilcoxon Rank Sum test, or, in case of multiple independent samples, the Kruskal-Wallis test.

All data are presented as mean \pm standard error of the mean (SEM), which is shown as error bars in the graphs. 


\subsection{Software and web tools}

\section{Software}

Image J

R Project

IGOR Pro

Web tools and databases

ClustalW

UniProt database

Vega database

Conserved Domain Search service

NetPhos 2.0
NIH, Bethesda, MD, USA

R Development Core Team (2009)

WaveMetrics Inc., Lake Oswego, OR, USA

Larkin et al. (2007)

UniProt Consortium (2010)

Ashurst et al. (2005)

Marchler-Bauer and Bryant (2004)

Blom et al. (1999) 


\section{3 \\ Expression of PSD-93 in the hippocampus}

The identity of the isoforms of PSD-93 expressed in the hippocampus is not known, but it might be a key step to infer the detailed function of this protein. The next five sections provide a comprehensive analysis about the isoform identity and thereby establish the basis for the functional characterization in Chapter 4 .

\subsection{Developmental profile}

As an initial step, the postnatal expression profile of PSD-93 in the hippocampus was compared to the expression profile of the other PSD-MAGUKs (Fig. 3.1A). In order to demonstrate that each lane contains the same amount of protein, the blot was probed for the presence of protein 14-3-3 $\beta$, a scaffolding protein shown to not vary during development (Umahara et al. 2009). As expected, protein 14-3-3 $\beta$ levels remain constant throughout development.

As reported previously, PSD-93 and PSD-95 showed similar temporal expression patterns (Funke 2007; Sans et al. 2000; Wang et al. 2006). Being weakly expressed in neonatal tissue, their expression increases sharply at two weeks after birth and reaches the plateau around three weeks after birth. For PSD-93 there is a total of four bands detected, all having similar temporal expression patterns (bands indicated by arrowheads). SAP97 is already present with $50 \%$ of its adult levels at P2 and thus does not change dramatically throughout development. This is in accord with Wang et al. 


\section{EXPRESSION OF PSD-93 IN THE HIPPOCAMPUS}

A

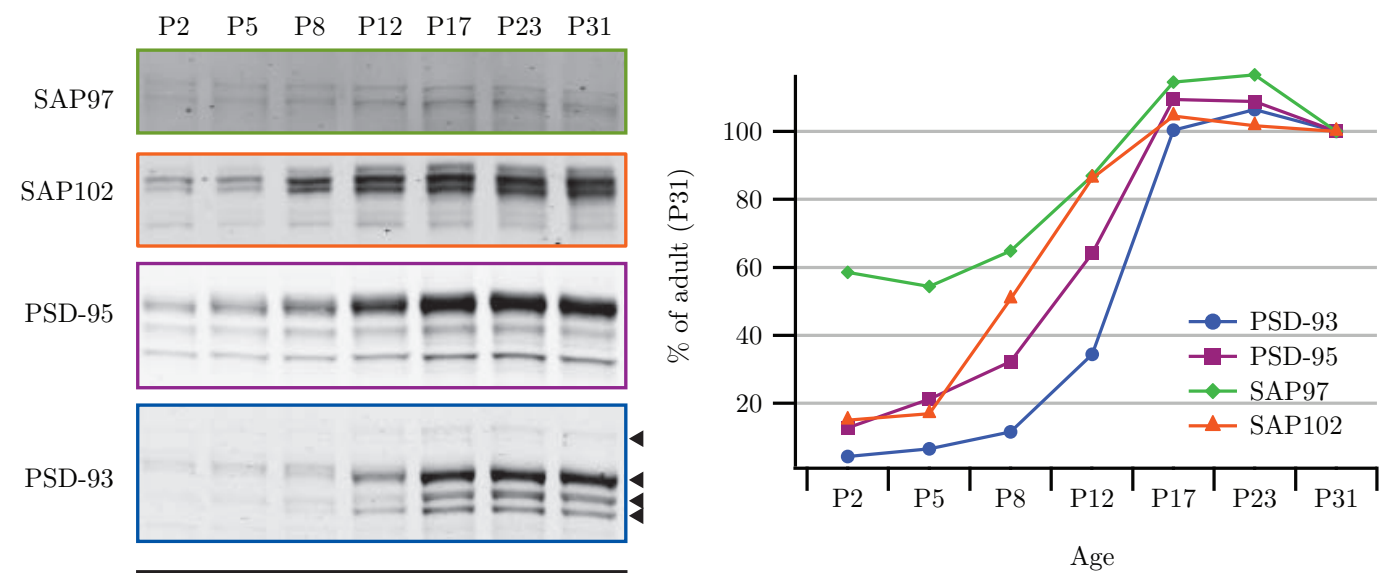

14-3-3 $\beta$

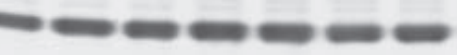

Age

B
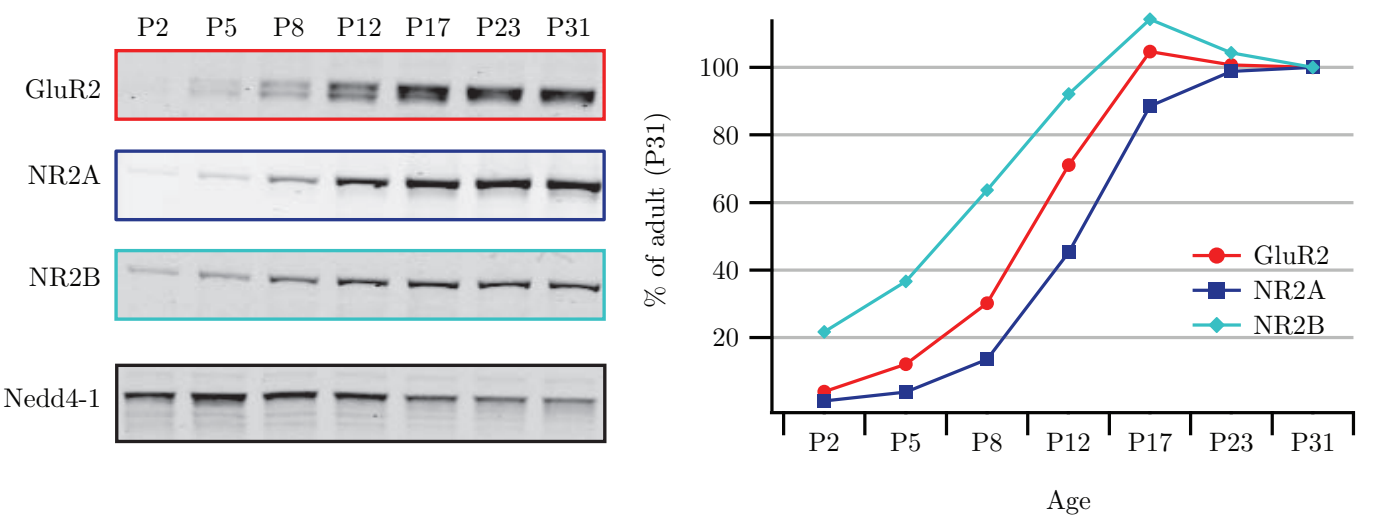

Figure 3.1: Developmental profile of PSD-MAGUKs and glutamate receptor subunits. Western blot of $\mathrm{S} 1$ fractions of hippocampal tissue from WT $(\mathrm{B} 6 \mathrm{~J} / \mathrm{N})$ mice of different ages. (A) Western blots were probed for PSD-MAGUK proteins. 14-3-3 $\beta$ is a scaffold protein that does not vary throughout development. The expression level of the PSD-MAGUKs were normalized to their respective adult (P31) expression level. (B) Western blots were probed for the glutamate receptor subunits GluR2, NR2A, and NR2B, and normalized to their respective adult (P31) expression level. Nedd4-1 is known to decrease throughout maturation.

(2006), who describe SAP97 as being expressed from E18 to P7 at constant levels, then increasing between P7 and P14 to reach adult levels. SAP102 expression levels are low 
until P5 and then increase sharply until P17 when they reach adult levels. In Funke (2007), SAP102 has been suggested to be high early in development and to decline from P15 on. Sans et al. (2000) on the other hand describe a profile mimicking the one shown in Figure 3.1.

The temporal expression profile of a selection of glutamate receptor subunits known to directly or indirectly bind to the PSD-MAGUKs is shown in Figure 3.1B. The AMPAR subunit GluR2 and the NMDAR subunit NR2A show expression patterns similar to those of PSD-95 and PSD-93. NR2B expression increases during development and shows a pattern similar to SAP102. NR2B expression peaks earlier than NR2A, fitting to the developmental switch from NR2B to NR2A (Liu et al. 2004).

Nedd4-1 is a neuronal precursor cell expressed and developmentally downregulated protein whose expression is highest from E12-P11 and then decreases dramatically until P22 (Kawabe et al. 2010). As shown in Figure 3.1B, Nedd 4-1 expression declines as expected.

\subsection{PSD-93 is alternatively spliced to generate multiple transcripts}

In mouse and rat, the PSD-93 gene is found on chromosomes 7 and 1, respectively. In mouse, the PSD-93 gene spans a region of almost 2 million nucleotides. Several isoforms have been described to exist (Brenman et al. 1996; El-Husseini et al. 2000c; Parker et al. 2004).

The PSD-MAGUKs have a common domain structure, and the isoforms mainly differ in the N-terminal domain. Across proteins, the N-termini show common features. PSD-95 and SAP97 both exist as an $\alpha$ - and a $\beta$-isoform. The $\alpha$-isoform contains a cysteine-doublet that is palmitoylated, whereas the $\beta$ - isoform has an L27 domain (Schlüter et al. 2006). Previously, the two isoforms of PSD-93 with N-terminal cysteines were named PSD-93 $\alpha$ and PSD-93 $\beta$, respectively. The isoform with an N-terminal L27

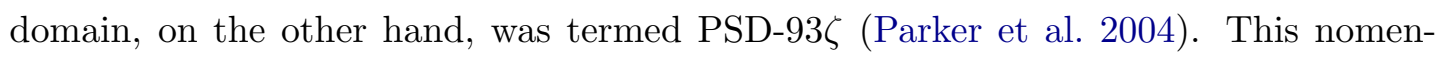
clature is very confusing, especially since the $\mathrm{N}$-termini might provide specific function to the isoforms as has been shown for PSD-95 and SAP97 (Schlüter et al. 2006). In this thesis, the nomenclature of the PSD-93 isoforms has been adapted to the scheme 


\section{EXPRESSION OF PSD-93 IN THE HIPPOCAMPUS}

Table 3.1: Nomenclature of the N-terminal splice variants of PSD-93.

This thesis Brenman 1996 El-Husseini 2000c Parker 2004 EST Acc. No.

\begin{tabular}{ccccc}
\hline$\alpha_{1}$ & $5 ’ \mathrm{~b}$ & $\alpha$ & $\alpha$ & $\mathrm{CJ} 130137$ \\
$\alpha_{2}$ & $5 ' \mathrm{a}$ & $\beta$ & $\beta$ & $\mathrm{BY} 123372$ \\
$\beta$ & - & - & $\zeta$ & $\mathrm{BB} 284284$ \\
$\gamma_{1}$ & - & - & - & AW060584 \\
$\gamma_{2}$ & $5 ' \mathrm{c}$ & - & $\gamma$ & AW060584 \\
$\delta$ & - & - & $\delta$ & $\mathrm{BY} 287980$ \\
$\epsilon$ & - & - & $\epsilon$ & - \\
\hline
\end{tabular}

of PSD-95 and SAP97, in analogy to Chetkovich et al. (2002). It is introduced in Table 3.1, where the respective counterpart of previous studies is also indicated.

Table 3.1 also shows a sample accession number of an expressed sequence tag (EST) clone. ESTs result from sequencing of mRNA from the 5' and 3' termini. Due to limitations in sequencing they are usually only 200-800 nucleotides long. However, these short bits of DNA have proven useful to determine genes expressed in certain cells or tissues as well as alternative splicing events.

Based on the ESTs, the exon-intron structure of PSD-93 was analyzed and is illustrated in Figure 3.2. The N-terminus of the $\beta$-isoform consists of five exons that are spliced onto exon 2. All other N-termini are encoded by a single exon. Exon 2 is common for the $\alpha$ - and $\beta$-isoforms and does not occur in the other isoforms since their start codon lies downstream of this exon. All isoforms apart from the $\beta$-isoform are spliced onto exon 3. From exon 4 on the isoforms are identical.

The N-terminus of the $\epsilon$-isoform was identified by screening a mouse superior cervical ganglion cDNA library (Parker et al. 2004). PSD-93€ does not contain any conserved domain. Yet, no EST clone was found to cover the N-terminus of PSD-93є.

The $\delta$-isoform of PSD-93 has a CxC motif similar to the $\alpha$-isoforms. This cysteine doublet is not at the immediate N-terminus but further downstream (amino acids 33 and 35). Still, the Conserved Domain Search service suggested that the cysteines are likely to be palmitoylated. In addition, a potential PEST sequence downstream of these 
cysteines was identified by the Conserved Domain Search service.

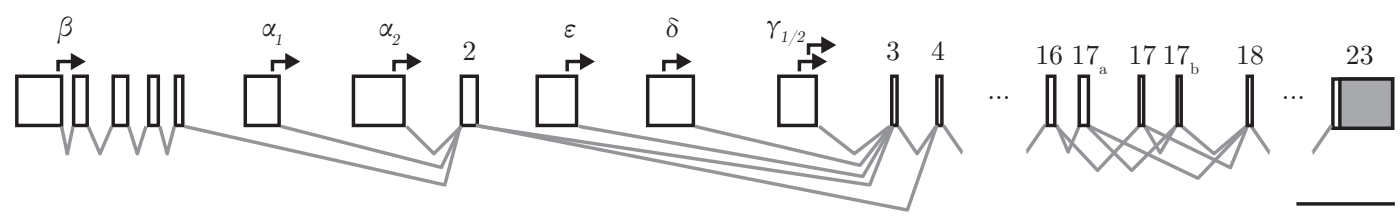

Figure 3.2: Exon-intron structure of PSD-93. The PSD-93 gene is equipped with six alternative promoters. The boxes indicate exons, which are drawn to scale (scale bar $1 \mathrm{~kb})$. Black arrows indicate the positions of the start codons, and the 3' UTR is color coded in gray.

The $\gamma$-isoform was described in the first paper on PSD-93 (Brenman et al. 1996), and was revisited by Parker et al. (2004). Nevertheless, it was never elaborated that this isoform potentially exists in a longer and/or a shorter version. The EST AW060584 describing the $\gamma$-isoform has two potential in frame start codons, none of which has a prominent Kozak sequence. The UniProt database describes the short isoform (Q91XM9-3), whereas another database, the Vertebrate Genome Annotation (Vega) database, describes the long isoform (dlg2-003). Since it is uncertain which isoform is endogenously expressed, I treated the two potential isoforms as being separate, calling the longer one PSD-93 $\gamma_{1}$, and the shorter one PSD-93 $\gamma_{2}$.

Alternative splicing also occurs at the C-terminus, between the SH3 and GK domains. In addition to the canonical database sequence in which exon 16 is followed by exon 17 and exon 18, two additional exons are described, which I termed exon 17a and 17b. Exon 17a precedes exon 17, and exon 17b follows exon 17.

Which splice variants occur on transcript level in the hippocampus is analyzed in the following two sections.

\subsubsection{N-terminal isoforms}

The N-terminal isoforms of PSD-93 differ in their 5' UTR and are thus likely to result from differential promoter usage. These promoters might be active in a tissuedependent manner. In order to get an idea which isoforms of PSD-93 are expressed in the hippocampus, I used semiquantitative RT-PCR. I wanted to see whether or not a respective isoform is present in hippocampal tissue and to compare its abundance to other tissues. A similar analysis has been performed by Leyland and Dart (2004) for 


\section{EXPRESSION OF PSD-93 IN THE HIPPOCAMPUS}

PSD-93 $\alpha_{1}, \alpha_{2}$ and $\delta$, but not for the other isoforms. Parker et al. (2004) extended the analysis to the other isoforms, but only compared brain against the superior cervical ganglion.

Some of the primers used for amplifying unique segments of the respective isoforms are identical to Parker et al. (2004), whereas others have been newly designed to optimize the specificity and product yield.

The mRNAs of all transcripts are present in the hippocampus, as shown in Figure 3.3A. It is important to note that the band intensity of the amplicons should not be compared across isoforms but rather across brain regions, since the primer characteristics might differ. The experiment was performed with two independent RNA samples for each brain region. The normalized integrated intensities of the two individual experiments is displayed as circles in Figure 3.3B. Apart from the experiment for PSD-93 $\gamma_{1 / 2}$, the results are highly reproducible. The bar graph (Fig. 3.3B) shows the average of the two experiments. One can see that the relative abundance of the transcripts for the six isoforms varies for different brain regions. The $\alpha$-isoforms are expressed to a much higher degree in the hippocampus compared to the cerebellum, whereas PSD-93 $\gamma_{1 / 2}$ is predominantly expressed in the cerebellum. The $\beta$-isoform is enriched in hippocampus, cortex and olfactory bulb, but is also present in the other brain regions. As shown previously, the PSD-93 $\delta$ expression level is the highest in the spinal cord, although the difference is not as dramatic as in the previous study (Leyland and Dart 2004). This might be due to the different primers used. PSD-93€ is expressed at similar levels in all brain regions studied.

\subsubsection{Splicing at the C-terminus}

In addition to the N-terminal splice variants, there is also variation of the transcript between the SH3 and GK domains. Screening EST clones, two additional exons were identified and termed exon 17a and exon 17b. Exon 17a shares about 80\% identity with the SAP97 exon I3, which is thought to be essential for the synaptic localization of SAP97 (Rumbaugh et al. 2003). The I3 insert provides an interaction domain for the neuronal actin/spectrin-associated protein 4.1N (Lue et al. 1994). Exon 17b has $90 \%$ sequence identity with the I5 insert of SAP97 and $60 \%$ with SAP102. Interestingly, it has been recently suggested that A-kinase anchoring protein 79/150 (AKAP79/150) 
A

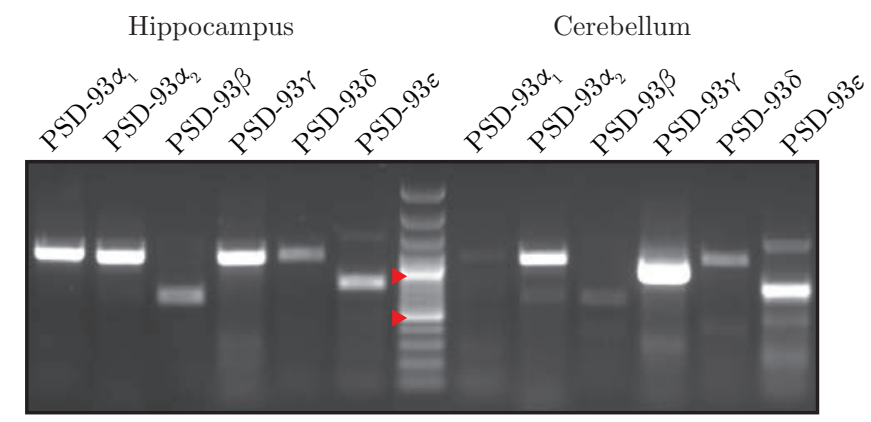

B

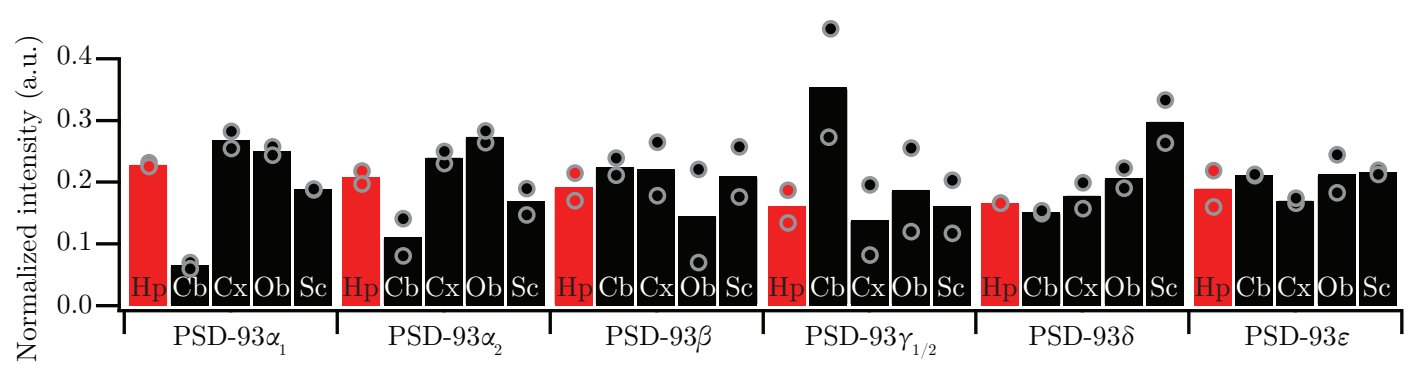

Figure 3.3: The mRNA of the different PSD-93 isoforms is present in many brain regions, but their abundance differs between tissues as detected by semiquantitative RT-PCR. (A) Example of RT-PCR products from hippocampus and cerebellum. 100bp ladder with arrowheads at $1 \mathrm{~kb}$ (top) and 500bp (bottom). Note that the intensity of the amplicons should not be compared across isoforms but rather across tissues. (B) Data of two independent RT-PCR experiments for different tissues and all PSD-93 isoforms. The average band intensity was calculated with ImageJ and compared across tissues. The intensity was normalized such that for one isoform the intensities sum up to one. The circles indicate the data of two independent experiments, and the bar graph their average. Abbreviations: Hp-hippocampus, Cb-cerebellum, Cx-cortex, Ob-olfactory bulb, Sc-spinal cord, a.u. arbitrary units. 


\section{EXPRESSION OF PSD-93 IN THE HIPPOCAMPUS}

specifically interacts with SAP97 containing both I3 and I5 inserts, and that this interaction is dynamically modulated by CaMKII $\alpha$ phosphorylation of SAP97 (Nikandrova et al. 2010).

Since it is not known in which combinations these exons exist in the hippocampus, I again used RT-PCR to analyze the presence of the transcripts.

A

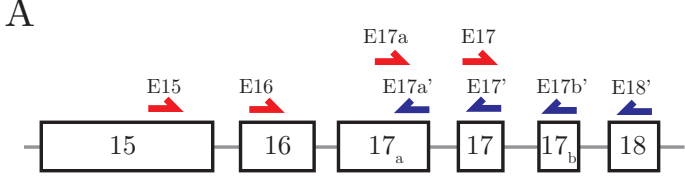

C

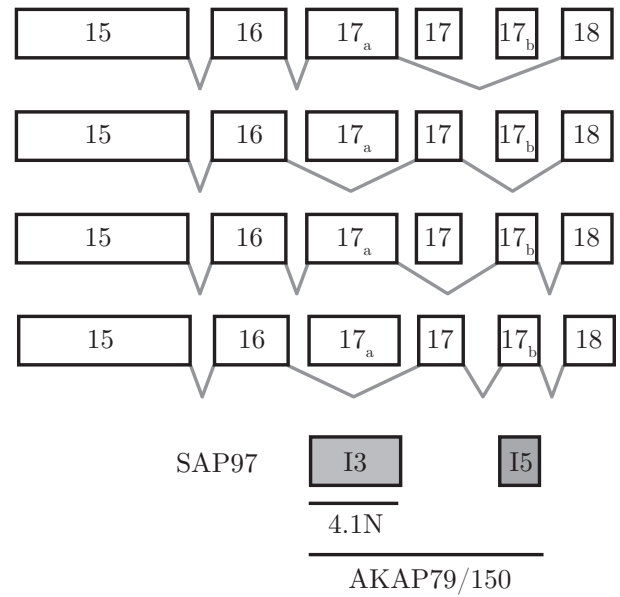

B1

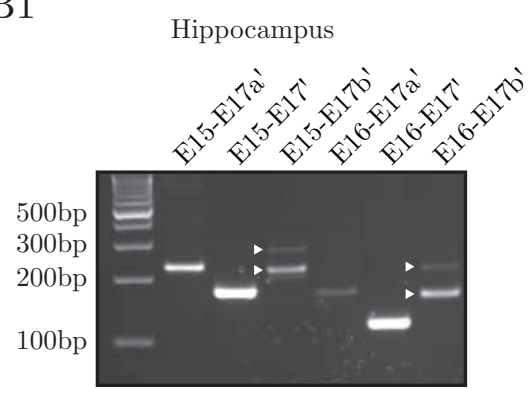

B2

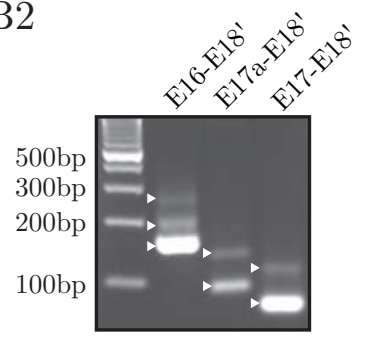

Figure 3.4: Splice variants between SH3 and GK domain. (A) Relative location of exon $17 \mathrm{a}$ and exon $17 \mathrm{~b}$ and primers used to analyze which isoforms are expressed. The exons are drawn to scale (scale bar 100nt). (B1, B2) Electrophoresis of the RTPCR products with arrowheads highlighting the product sizes discussed in the text. (C) Combinations of exons expressed in the hippocampus as deduced from B1, B2. Exons are drawn to scale as in A. The homologous exons of SAP97 are indicated at the bottom in gray, together with their identified interaction partners.

As can be seen in Figure 3.4B1, exon 16 is not an alternatively spliced exon. The first three lanes are identical to the last three lanes, just shifted 76nt upward. This shift fully accounts for the size of exon 16. Lane four shows that the combination 16-17a exists (expected size 165nt). Lane five indicates that exon 17 never occurs in combination with exon $17 \mathrm{a}$, since the expected size for the combination 16-17a- 
17 is $215 \mathrm{nt}$, and that of $16-17$ is $115 \mathrm{nt}$. Lane six shows that exon $17 \mathrm{~b}$ does occur in combination with exon 17a or exon 17, but not alone and also not with both of them in the same transcript (expected sizes: 16-17b 112nt, 16-17a-17b 212nt, 16-1718b 158nt, 16-17a-17-17b 258nt). Figure 3.4B2 demonstrates that all the isoforms mentioned before are spliced onto exon 18. The PCR product in lane one shows three bands, fitting to the four combinations 16-17a-18 (217nt), 16-17-18 (163nt), 16-17a17b-18 (259nt), and 16-17-17b-18 (205nt). Lane two demonstrates that 17a-18 (94nt) and 17a-17b-18 (136nt) exist in the hippocampus. Finally, lane three shows a major band for 17-18 (80nt) and another band for 17-17b-18 (122nt).

All transcripts present in the hippocampus for this splice site are summarized in Figure 3.4C. In conclusion, $17 \mathrm{a}$ and 17 are mutually exclusive exons, and exon $17 \mathrm{~b}$ is a so-called cassette exon; it is either spliced in after exon 17 a or exon 17 , or excluded from the transcript.

Of course, the splicing at the C-terminus introduces a high degree of complexity. The six N-terminal and the four C-terminal splice variants can theoretically yield 24 different proteins. For PSD-95 and SAP97 it has been shown that the $\alpha$ - and $\beta-\mathrm{N}$ termini completely change the protein's function. Since it is not known what function the N-terminal splice variants of PSD-93 provide, the following analysis of PSD-93 expression and function focusses on the $\mathrm{N}$-terminal domains as they likely provide more functional differences. All constructs used in this study thus contain the canonical Cterminal exon combination 16-17-18.

\subsection{Tools to study PSD-93 expression and function}

In order to study the isoform identity of PSD-93 in hippocampal synapses and to analyze its function, I wanted to combine knockdown of the endogenous protein with overexpression of specific isoforms of PSD-93. For this, efficient RNA interference sequences had to be found and overexpression constructs cloned.

In the following paragraphs, the RNAi constructs are introduced and their efficiency is demonstrated. With the use of protein biochemistry and mass spectrometry, I can define a subset of isoforms that is definitely expressed in the hippocampus. Besides, I can estimate the relative amount of endogenous PSD-93 in the hippocampus. 


\section{EXPRESSION OF PSD-93 IN THE HIPPOCAMPUS}

\subsubsection{Knockdown constructs for PSD-93}

RNA interference can be used to silence protein synthesis at the post-transcriptional level. Double-stranded RNA is introduced into cells and either leads to a sequencespecific degradation of complementary mRNA or to a block of its translation. Thus, RNAi enables the study of loss-of-function of individual genes. Compared to knockout strategies, RNAi is more rapid, cost-effective and permits temporally and spatially controlled gene expression.

One challenge of RNAi studies is to deliver the knockdown construct into the cells of interest. While dividing cell lines like HEK293 cells can be easily transfected, this is different for neurons. Therefore, we use lentivirus to transduce cultured neurons as illustrated in Figure 3.5. The lentivirus vector contains an expression cassette for a short-hairpin RNA (shRNA), driven by RNA pol III promoters. These promoters have the advantage of efficiently directing the synthesis of small, non-coding transcripts with well-defined ends and are thus ideal for shRNAs. The lentivirus vector stably recombines into the host genome and permanently expresses the RNAi construct.

In principle, any gene can be silenced, but it is not always easy to find a target sequence that leads to an efficient knockdown of at least $85 \%$ of the protein. Four different RNAi constructs for PSD-93 were used in this study. FUrn931UGW (rn931) and FUrn932UGW (rn932) have been described in Elias et al. (2006). FHsh93dUGW (sh93d) was used in Ogawa et al. (2008). The target sequence used for FU6tn93bUGW (tn93b) was predicted by The RNAi Consortium (Moffat et al. 2006) and also received a high score with a self-made scoring system. This sequence was constructed as a 29mer (Siolas et al. 2005), driven by the human U6 promoter. sh93d is driven by the H1 promoter, and the other two are driven by the mouse U6 promoter (rn931, rn932).

Because rat and mouse sequences may have considerable differences on the transcript level, different RNAi constructs can have variable efficiency in different species. As shown in Figure 3.6A, sh93d and tn93b efficiently reduce PSD-93 protein levels in dissociated hippocampal cultures from mice, whereas the constructs rn931 and rn932 do not. On the other hand, rn931 and rn932 as well as to a lower degree sh93d efficiently reduce protein expression in rat hippocampal cultures (Fig. 3.6B). However, tn93b shows hardly any knockdown of rat PSD-93. 


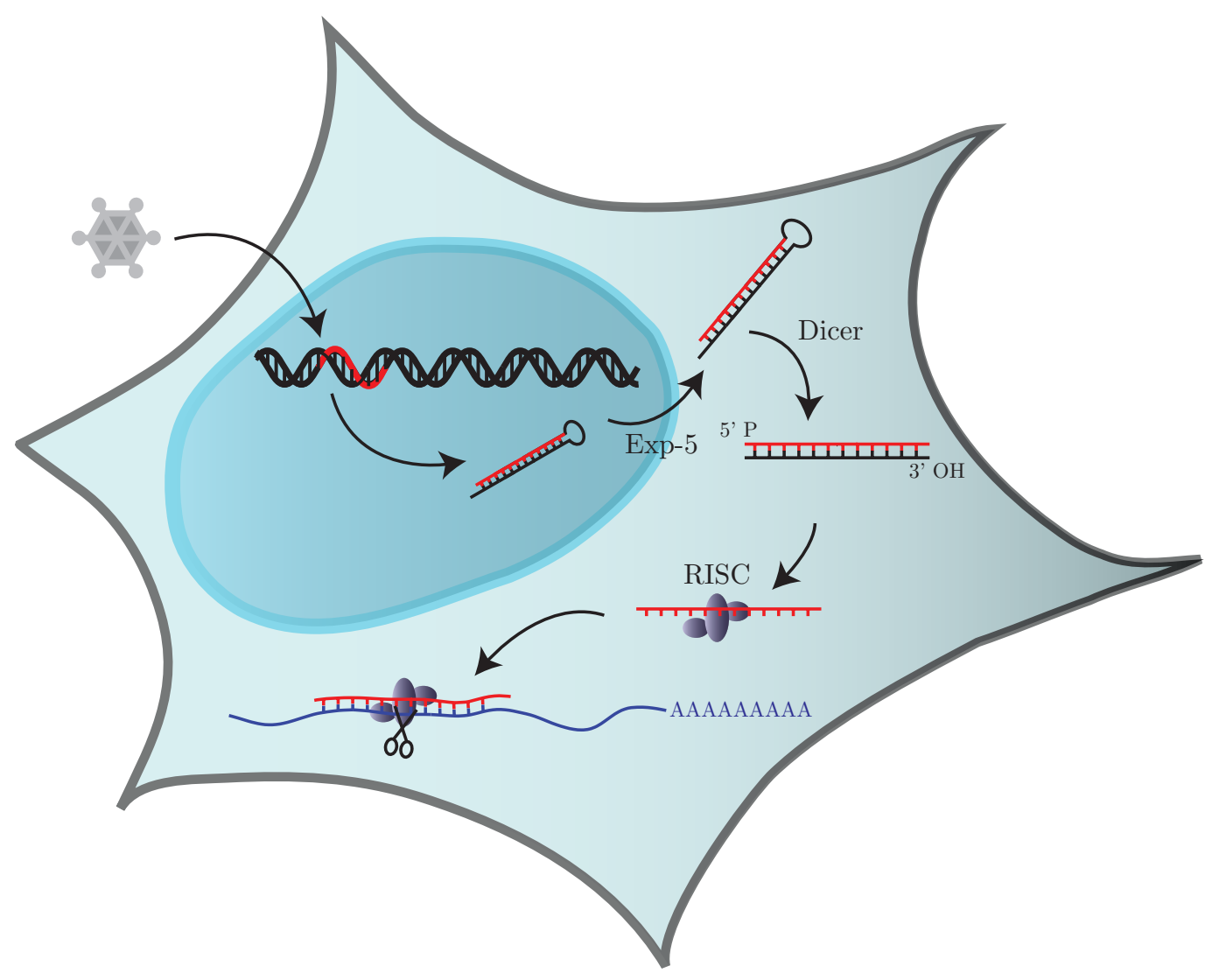

Figure 3.5: Lentiviral transduction of cells to drive shRNA expression. When a cell is infected by a lentivirus carrying an shRNA expression cassette, the expression cassette stably recombines into the cell's genome. A small RNA is transcribed from this expression cassette and folds into a hairpin structure. This hairpin mimics endogenous microRNAs and is supposedly analogously exported out of the nucleus by the Exportin-5Ran-GTP complex. In the cytoplasm, the hairpin is processed by the RNase Dicer, and one strand of the RNA duplex is incorporated into the RNA-induced silencing complex (RISC). This protein-RNA complex binds to complementary mRNA and either results in mRNA degradation or block of translation.

Importantly, knockdown of PSD-93 does not unspecifically knockdown PSD-95 (Fig. 3.6B). The expression of the glutamate receptor subunits NR2B and GluR2 is also not affected by knockdown of PSD-93. As well, the expression of the NMDAR subunit NR2A is unchanged (data not shown). In Figure 3.6C, the results of several knockdown experiments with mouse and rat hippocampal cultures are summarized as mean knockdown \pm SEM (N, number of experiments). 
A

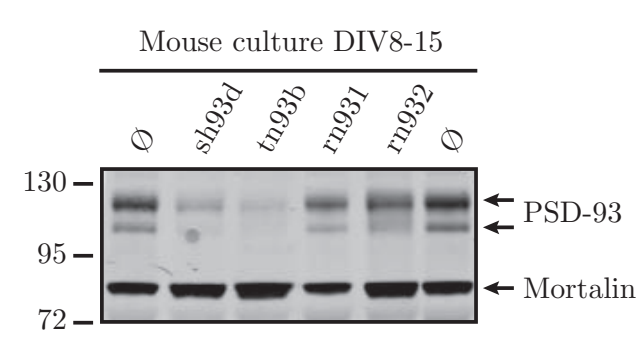

B

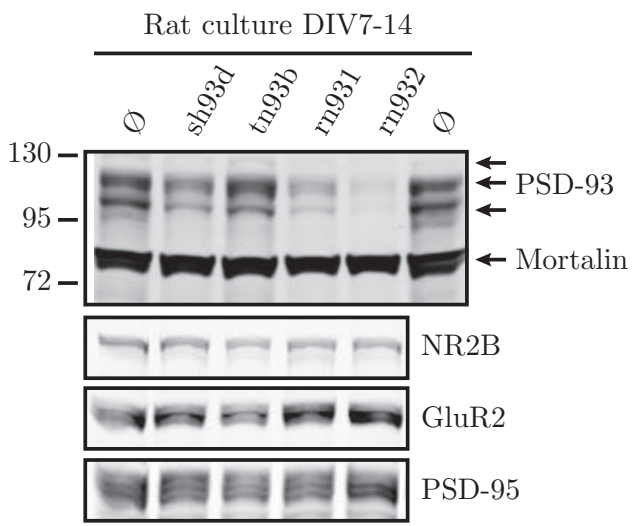

$\mathrm{C}$

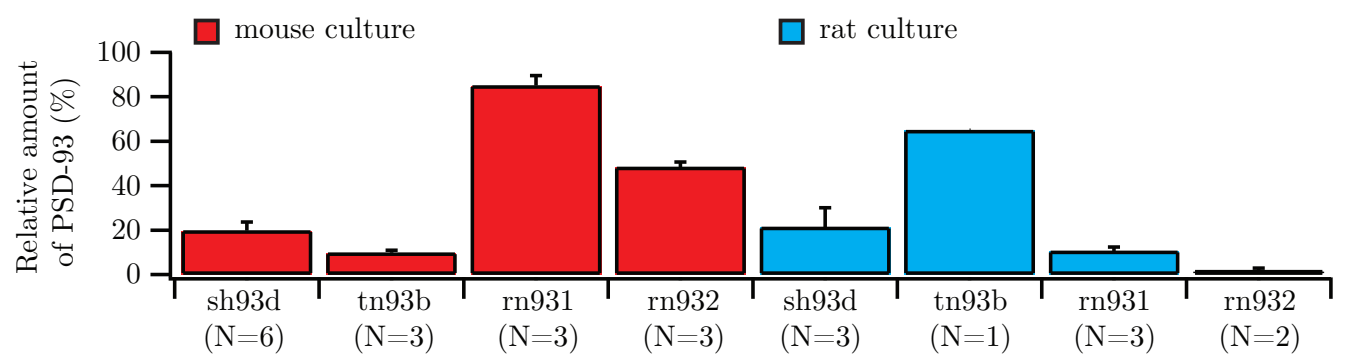

Figure 3.6: Knockdown constructs for PSD-93 have differential efficiency in mouse and rat. (A) Western blot of mouse cultures infected with the RNAi constructs sh93d, tn93b, rn931 and rn932. Mortalin is used to normalize the signal to the amount of protein per lane. (B) Western blot of rat cultures infected with the same RNAi constructs as in (A). The expression of PSD-95, as well as of GluR2 and NR2B is not affected by RNAi-mediated knockdown of PSD-93. (C) Quantification of the knockdown efficiency in mouse and rat based on several experiments (N, number of experiments).

\subsubsection{Antibody characterization}

Unfortunately, antibodies for the specific isoforms of PSD-93 do not exist. Instead, I had access to different antibodies with different characteristics, which are described in more detail below.

\subsubsection{Monoclonal PSD-93 antibody}

The monoclonal PSD-93 antibody (NeuroMab) has roughly the same affinity for all isoforms of PSD-93. Recombinant GFP-fusion proteins of the different isoforms were 
expressed in neurons and the samples subjected to SDS-PAGE and immunoblotted. The same membrane was probed with $\alpha$ - PSD-93 (mouse, ms) and $\alpha$ - GFP (rabbit, $\mathrm{rb}$ ). The secondary antibodies were coupled to different infrared dyes such that they could be simultaneously detected in the $700 \mathrm{~nm}$ or $800 \mathrm{~nm}$ channel, respectively. As demonstrated in Figure 3.7A, PSD-93 $\alpha_{1}$ is detected with the highest affinity. This is not surprising, as this antibody is raised against the amino acids 1-852 of rat PSD-93 $\alpha_{1}$ (UniProt ID Q63622). PSD-93 $\alpha_{2}$ is detected with 90\%, PSD-93 $\beta$ with $64 \%$, PSD-93 $\gamma_{1}$ with $74 \%$, PSD-93 $\gamma_{2}$ with $76 \%$, PSD-93 $\delta$ with $81 \%$, and PSD-93 $\epsilon$ with $93 \%$ affinity.

Unfortunately, this antibody shows some cross-reactivity with PSD-95 at high concentrations of the antigen. When PSD-95 is overexpressed as a GFP-fusion protein, a high-molecular weight band is detected which is also detected by $\alpha$-GFP and which is still present in case of acute knockdown of PSD-93 with sh93rn2 (Fig. 3.7B).

A

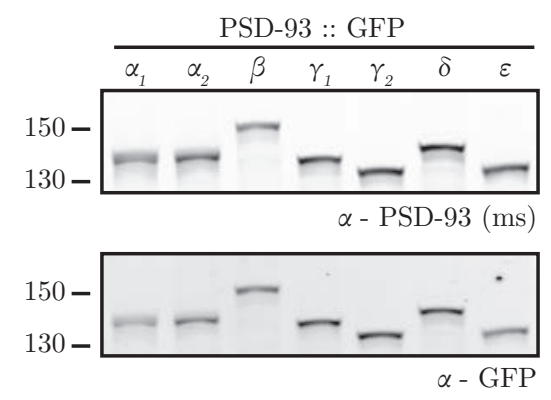

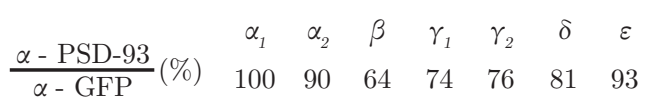

$\mathrm{B}$

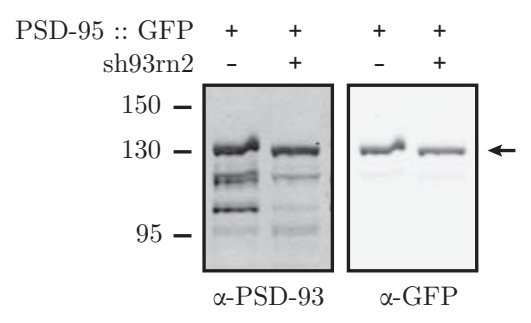

Figure 3.7: Characterization of the monoclonal antibody for PSD-93. (A) $\alpha$ PSD-93 (ms) detects all isoforms with almost the same affinity. (B) m $\alpha$-PSD-93 shows cross-reactivity with PSD-95.

\subsubsection{Polyclonal PSD-93 antibody}

The same characterization was performed for the polyclonal PSD-93 antiserum raised in rabbit and kindly provided by Dr. David Bredt. Recombinant GFP-fusion proteins of the different isoforms were expressed in neurons and the samples were immunoblotted with $\alpha$-PSD-93 (rb) and $\alpha$-GFP (ms). Again, the secondary antibodies were coupled 


\section{EXPRESSION OF PSD-93 IN THE HIPPOCAMPUS}

to different infrared dyes, allowing the simultaneous detection in separate channels (Fig. 3.8). According to Sans et al. (2000), the polyclonal PSD-93 antibody is raised against amino acids 299-417 of PSD-93, a region conserved among the different isoforms. Hence, it is not surprising that the affinity for the different isoforms is similar. PSD$93 \alpha_{1}, \alpha_{2}, \beta$ and $\epsilon$ are detected with $100 \%$ affinity, PSD-93 $\gamma_{1}$ with $73 \%, \gamma_{2}$ with $86 \%$ and $\delta$ with $77 \%$.

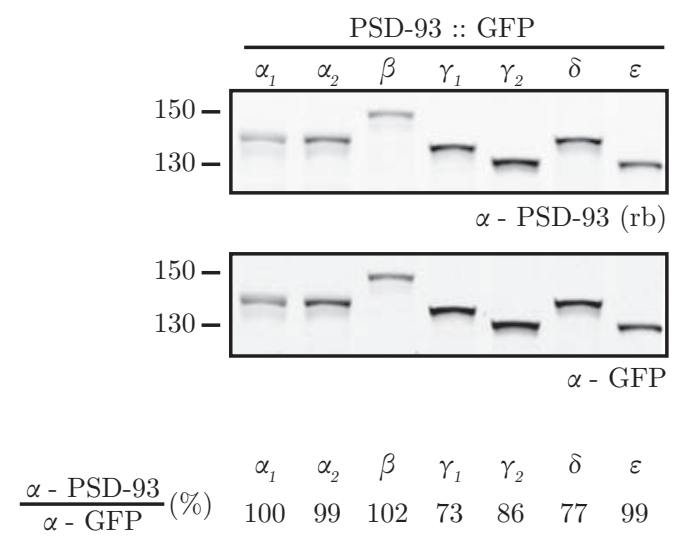

Figure 3.8: Characterization of the polyclonal antibody for PSD-93. $\alpha$-PSD-93

$(\mathrm{rb})$ detects all isoforms with roughly the same affinity.

\subsubsection{3 $\alpha / \beta$-MAGUK antibody}

There is a commercially available antibody for SAP97 (Assay Designs). Its immunogen corresponds to the N-terminal amino acids 1-163 of rat SAP97 $\beta$ including exon 2. Exon 2 has a segment which is conserved among the $\alpha$ - and $\beta$-isoforms of the PSDMAGUKs (Fig. 3.9). Hence, this antibody has been used previously to detect the $\alpha$ and $\beta$-isoforms of PSD-95 and SAP97 (Schlüter et al. 2006), and might therefore also detect PSD-93 $\alpha$ and PSD-93 $\beta$.

In order to characterize the affinity of this antibody for the members of the PSDMAGUK family and to confirm the specificity for the $\alpha$ - and $\beta$-isoforms of PSD-93, dissociated neuronal cultures were used to overexpress GFP-fusion proteins of the individual isoforms. As shown in Figure 3.10A, only PSD-93 $\alpha_{1}$, PSD-93 $\alpha_{2}$ and PSD-93 $\beta$ are detected by this antibody. However, endogenous PSD-93 seems to be also detected by this antibody, in contrast to what has been shown previously (Schlüter et al. 2006). 


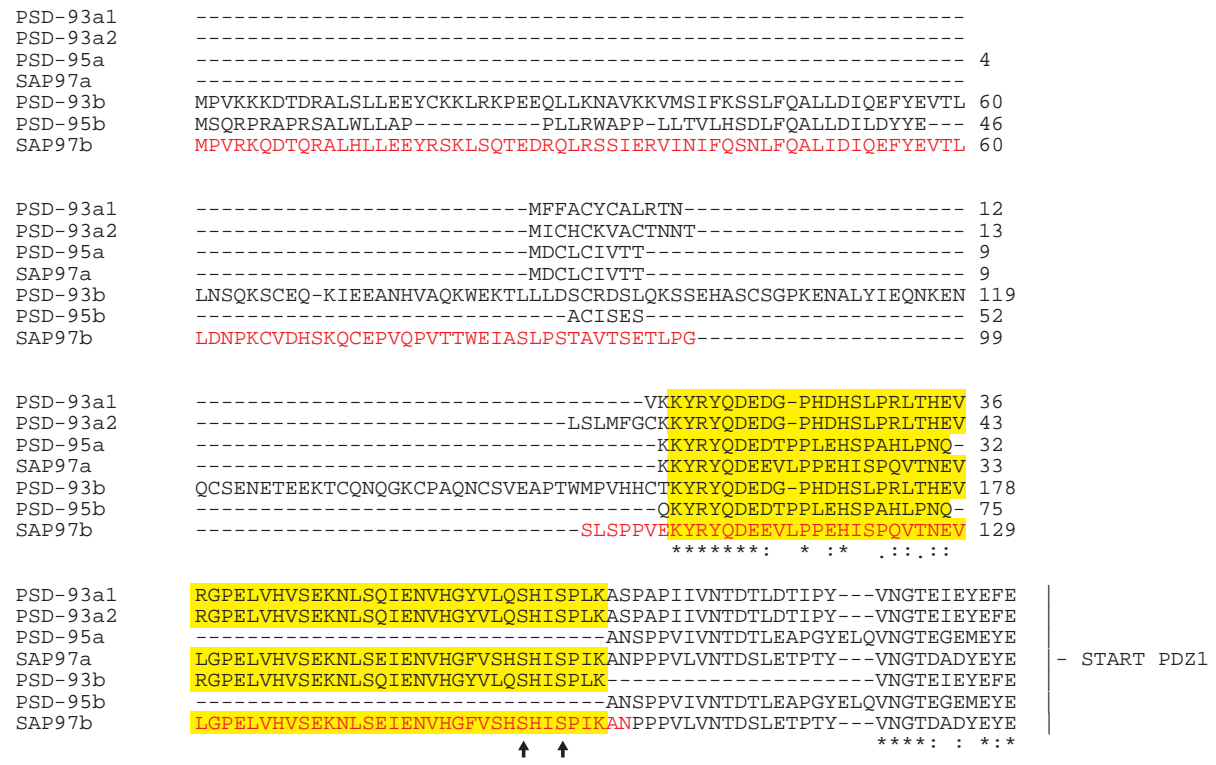

Figure 3.9: At the N-terminus of the PSD-MAGUKs there are conserved regions. Alignment of the $\alpha$ - and $\beta$-isoforms of PSD-93, PSD-95 and SAP97. The immunogen for $\alpha$-SAP97 is shown in red. The yellow area indicates the exon 2 of PSD-93, PSD-95 and SAP97, which, as can be appreciated, is partially conserved. The two arrows mark potential phosphorylation sites to be discussed on page 96 .

I therefore compared the current lot (Lot No. B505457) of this antibody to the lot that was used in the previous study (Lot No. 04190610) (Schlüter et al. 2006). Indeed, this new lot of the antibody detects PSD-93 with high affinity in PSD-93 WT but not in $\mathrm{KO}$ mice (Fig. 3.10B). In contrast, using the old lot of the antibody, only a very faint band appears for PSD-93. Thus, the conclusion drawn in the study by Schlüter et al. (2006) that PSD-93 $\alpha_{1 / 2}$ and $\beta$ are absent from the hippocampus does not hold true in the light of the new antibody lot. PSD-93 $\alpha_{1}$, PSD-93 $\alpha_{2}$, and/or PSD-93 $\beta$ are endogenously expressed in the hippocampus.

PSD-95 and SAP97 are not upregulated in the PSD-93 KO animals. In fact, the observed increase in expression level in Figure 3.10B results from a higher total protein concentration in the KO sample compared to the WT. Probing the western blot with an antibody against GAPDH (glyceraldehyde-3-phosphate dehydrogenase, a protein thought to be present at constant levels), it becomes apparent that the KO sample contains more GAPDH and hence more total protein. 


\section{EXPRESSION OF PSD-93 IN THE HIPPOCAMPUS}

A
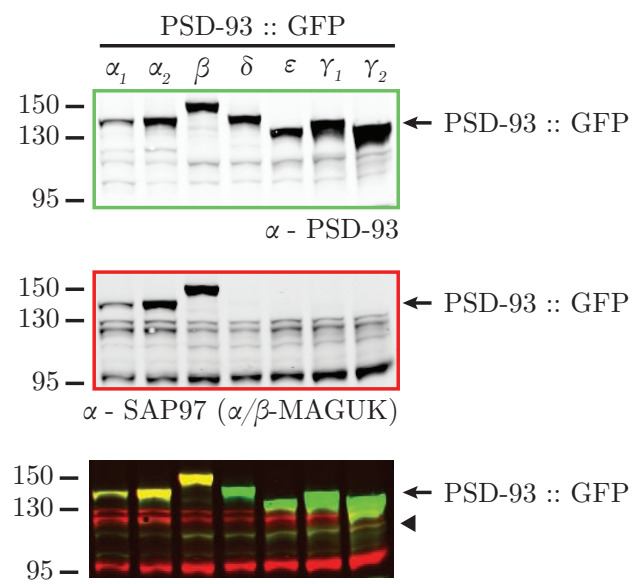

B
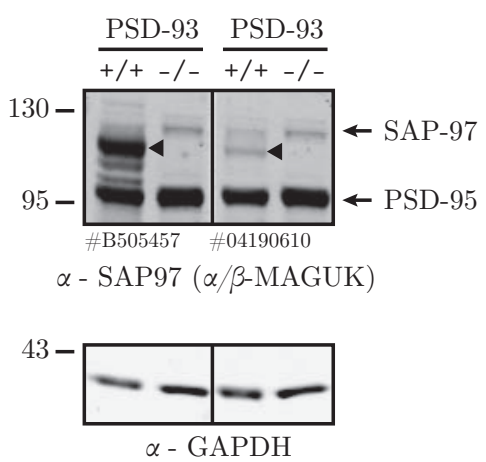

Figure 3.10: $\alpha$-SAP97 detects only the $\alpha$ - and $\beta$-isoforms of PSD-93. (A) Top: PSD-93-GFP fusion proteins are detected by the monoclonal PSD-93 antibody. Also, endogenous PSD-93 is detected as faint bands. Middle: $\alpha$-SAP97 detects the GFP-fusion proteins of PSD-93 $\alpha_{1}$, PSD-93 $\alpha_{2}$ and PSD-93 $\beta$, as well as endogenous SAP97 (double band $\sim 130 \mathrm{kDa}$ ), PSD-95 ( 95kDa) and PSD-93 (faint bands $\sim 110 \mathrm{kDa})$. Bottom: Merge of the two images above, yellow indicating overlay. Arrowhead points at endogenous PSD93, which is detected by both antibodies. (B) The affinity of two different lots of $\alpha$ SAP97 (AssayDesigns) was compared in WT and PSD-93 KO mice. Whereas the new lot (B505457) of the antibody shows a strong signal for PSD-93, there is only a faint band with the old lot (04190610) of the antibody. The observed increase of PSD-95 and SAP97 levels in the KO compared to the WT is due to a higher protein concentration of the KO as shown with the housekeeping protein GAPDH (bottom).

In order to see whether there is a different affinity of $\alpha$-SAP97 towards the different PSD-MAGUKS, I overexpressed PSD-93, PSD-95, and SAP97 in dissociated hippocampal cultures. I normalized the expression levels with $\alpha$-GFP (rb) and then probed with $\alpha$-SAP97. As seen in Figure 3.11A, $\alpha$-SAP97 has different affinities for the different antigens. This is quantified in Figure 3.11B. Obviously, the antibody affinity is highest for SAP97 $\beta$ (100\%), and surprisingly followed by PSD-95 $\alpha$ (84\%). PSD-93 $\beta$ is detected with $45 \%$ and PSD-93 $\alpha_{1}$ and PSD-93 $\alpha_{2}$ with $22 \%$ and $25 \%$ affinity, respectively. 
A
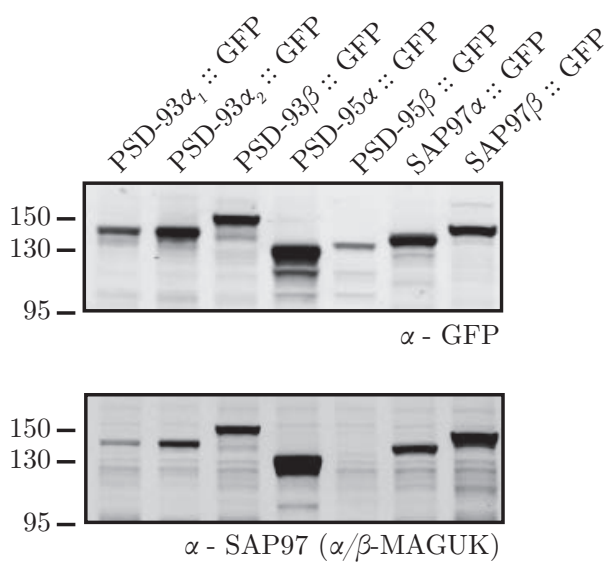

B

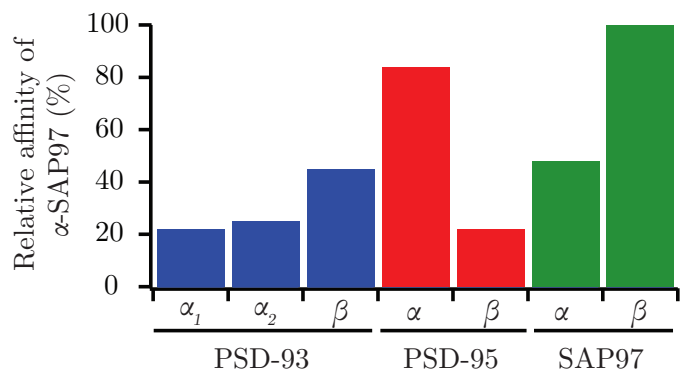

Figure 3.11: $\alpha$-SAP97 has a different affinity for PSD-95, PSD-93 and SAP97. (A) Example of a western blot of overexpression constructs of the $\alpha$ - and $\beta$-isoforms of PSD93, PSD-95 and SAP97. $\alpha$-GFP (rb) shows the absolute levels of the recombinant proteins, and $\alpha$-SAP97 the affinity of the antibody towards the antigens. SAP97 $\beta$ is detected with the highest affinity, PSD-95 $\beta$ with the lowest. (B) Quantification of N=2 western blots.

\subsubsection{Identification of PSD-93 isoforms expressed in the hippocampus}

\subsubsection{Analyzing the molecular weight of the isoforms}

Figure 3.1A suggests that several isoforms of PSD-93 are expressed in the hippocampus since four bands appear on the western blot when probed with an $\alpha$-PSD-93 antibody. From the RT-PCR experiments, none of the N-terminal splice variants can be excluded from being expressed in the hippocampus. Thus, in order to elucidate the bands' identities, recombinant full-length PSD-93 isoforms were cloned and expressed in hippocampal dissociated cultures of mouse. For this, bicistronic constructs driving expression of a specific isoform of PSD-93 from the first cistron, and the expression of GFP from the second cistron were used. These constructs are based on the rat coding sequence (see p.18). Simultaneously, endogenous PSD-93 was knocked down in the cultures using the RNAi construct tn93b, which has been shown to efficiently knockdown mouse but not rat PSD-93 (Fig. 3.6).

The PSD-93-IRES-GFP samples were subjected to SDS-PAGE, together with samples from hippocampal tissue as used in Figure 3.1. In Figure 3.12, the first lane is 


\section{EXPRESSION OF PSD-93 IN THE HIPPOCAMPUS}

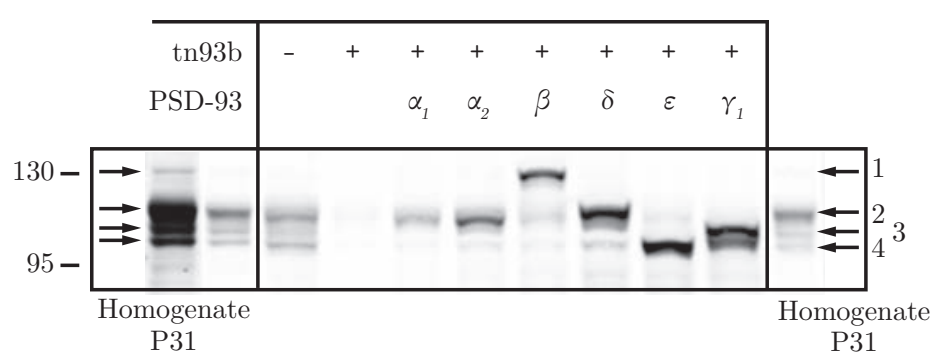

Figure 3.12: Expressing untagged PSD-93 shows that PSD-93 $\beta$, PSD-93 $\alpha_{1 / 2}$, PSD-93 $\gamma_{1}$ are expressed in the hippocampus. Murine hippocampal cultures were infected with the knockdown construct tn93b that specifically works in mouse but not in rat. Simultaneously, untagged PSD-93 isoforms were overexpressed to observe their molecular weight and compare it with homogenate. The left-most lane is a contrast enhanced version of the second lane. PSD-93 $\beta$ corresponds to the highest band. PSD-93 $\alpha_{1 / 2}$ corresponds to the second band as this also showed reactivity with the $\alpha / \beta$-MAGUK antibody. $\gamma_{1}$ might be responsible for the lower two bands. However, the presence of PSD-93 $\epsilon$ and $\delta$ cannot be excluded.

a duplication of the second lane but contrast enhanced such that the upmost band becomes visible. As one can see, the upmost band appears to be PSD-93 $\beta$. The second band runs on the same height as PSD-93 $\alpha_{1}$, PSD-93 $\alpha_{2}$, and PSD-93 $\delta$. Since this band showed immunoreactivity with the $\alpha / \beta$-MAGUK antibody (see Fig. 3.10A and B), it follows that PSD-93 $\alpha_{1}$ and/or PSD-93 $\alpha_{2}$ is expressed in the hippocampus. Whether PSD-93 $\delta$ is present cannot be derived from this experiment, but will be investigated in the following section.

PSD-93 $\gamma_{1}$ results in two bands, one being full-length PSD-93 $\gamma_{1}$ and the other one potentially a degradation product. The upper band runs on the same height as the third PSD-93 band, whereas the truncated form runs on the height of the fourth band. Thus, PSD-93 $\gamma_{1}$ might be responsible for both lower molecular weight bands. N-terminal truncation has been described for PSD-95 $\alpha$ (Xu et al. 2008), and there might be a similar mechanism for PSD-93 $\gamma_{1}$. The lowest running band in hippocampal homogenate, however, might also result from PSD-93e, which runs at this size. PSD-93 $\gamma_{2}$ was not cloned as a bicistronic construct and hence could not be tested. 


\subsubsection{Traces of PSD-93 $\delta$ are expressed in the hippocampus}

PSD-93 $\alpha_{1 / 2}$ and PSD-93 $\delta$ have the same molecular weight and thus cannot be differentiated on a western blot. With antiserum raised against amino acids 1-20 of PSD-93 that was kindly provided by Dr. Mark Leyland (Leyland and Dart 2004), I tested the presence of PSD-93 $\delta$ in the hippocampus. In order to verify that this antiserum is specific, I overexpressed PSD-93 $\alpha_{1}$, PSD-93 $\alpha_{2}$ and PSD-93 $\delta$ in dissociated cultures of rat hippocampal neurons and immunoprecipitated all isoforms with the monoclonal PSD-93 antibody and Protein G-coupled sepharose beads. As shown in Figure 3.13A, only PSD-93 $\delta$ and not the $\alpha$-isoforms are detected by the antiserum.

Having a tool at hands to determine the proportion of PSD-93 $\delta$ in the hippocampus, I asked how much of the immunoprecipitate of PSD-93 is the $\delta$-isoform. I could not test homogenate or P2 fraction because the antiserum showed a high degree of crossreactivity. Therefore, I probed the immunoblot of the IP of PSD-93 with the PSD$93 \delta$-antiserum (Fig. 3.13B). Immunoprecipitated PSD-938::GFP was used to normalize the PSD-93 $\delta$-antiserum and the monoclonal PSD-93 antibody. It appears that only $5 \%$ of this band is contributed by PSD-93 $\delta$, the remaining $95 \%$ is PSD- $93 \alpha_{1}$ and/or PSD-93 $\alpha_{2}$.

\subsubsection{Quantification of PSD-93 and PSD-95}

Complementary to the quantification of PSD-93 in the last paragraph, the antibodies and overexpression constructs for PSD-95 and PSD-93 enable me to quantify the relative amount of endogenous PSD-93 and PSD-95 protein present in hippocampal homogenate (H). To this end, PSD-93 $\alpha_{1}$ and PSD-95 $\alpha$ were overexpressed in HEK293T cells as GFP-fusion proteins. They were harvested and separated by SDS-PAGE together with homogenate and P2 fraction samples of hippocampal tissue of adult mice. The samples were western blotted and probed with the polyclonal $\alpha$-GFP antibody, as well as either the monoclonal $\alpha$-PSD-93 or $\alpha$-PSD-95 antibody.

As shown on the left hand side in Figure 3.14A, $\alpha$-GFP detects similar amounts of the GFP-fusion proteins of PSD-93 and PSD-95. The affinity of $\alpha$-PSD-95 is approximately 1.2-fold higher than of $\alpha$-PSD-93. With this information at hands, the amount of PSD-93 (three bands as indicated by red arrowheads, PSD-93 $\beta$ is not visible) in the 


\section{EXPRESSION OF PSD-93 IN THE HIPPOCAMPUS}

A

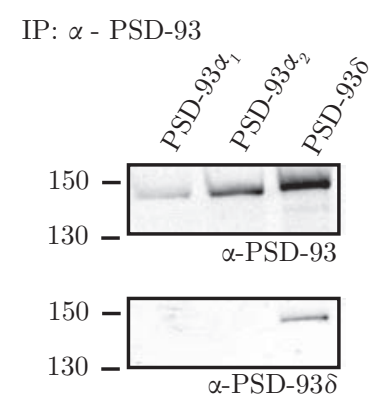

B

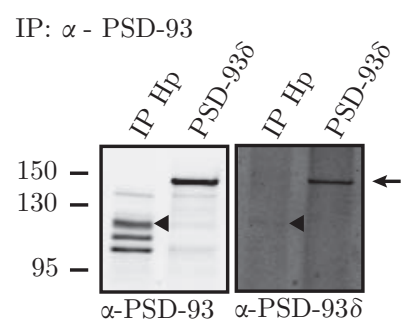

Figure 3.13: PSD-93 $\delta$ only makes up at most $5 \%$ of PSD-93 in the hippocampus. (A) PSD-93 $\alpha_{1}::$ GFP, PSD-93 $\alpha_{2}::$ GFP and PSD-93 $::$ GFP were immunoprecipitated from dissociated cultures. All are detected by the monoclonal PSD-93-antibody, but only $\delta$ is detected by the PSD-93 $\delta$-antiserum. (B) Left lane: IP of PSD-93 isoforms from hippocampal tissue showing 4 bands. The second band from top might contain some PSD-93 Right lane: Overexpression of PSD-93 $:$ GFP in hippocampal cultures and IP with $\alpha$-PSD93 (ms). PSD-93 ::GFP is detected by $\alpha$-PSD-93 and $\alpha$-PSD-93 $\delta$ (arrow). There is a faint band of PSD-93 in the IP from hippocampal tissue that runs on the right height (arrowheads). However, comparing the signals of the two antibodies, this accounts for only $5 \%$ of this band.

tissue sample of mouse hippocampus (homogenate and P2 fraction) can now be quantified as $H_{93}=h_{93} * \frac{r_{G F P}}{r_{93}}$, with the densitometrically analyzed intensities of the bands of $\alpha$-PSD-93 in the homogenate $\left(h_{93}\right), \alpha$-PSD-93 of the recombinant PSD-93::GFP fusion protein $\left(r_{93}\right)$, and $\alpha$-GFP for the recombinant PSD-93::GFP fusion protein $\left(r_{G F P}\right)$. The same calculation was done for PSD-95. From this, the total amount of PSD-93 appears to be $40 \%$ of PSD-95 in the homogenate, and 35\% in the P2 fraction.

A similar estimate can be derived with the $\alpha / \beta$-MAGUK antibody for the $\alpha$ isoforms of PSD-93. Again, the recombinant protein expressed in HEK293T cells was analyzed on the same western blot as samples from hippocampal homogenate and P2 fraction. The membrane was probed with the polyclonal $\alpha$-GFP antibody and $\alpha$ SAP97 ( $\alpha / \beta$-MAGUK, Lot No. B505457). In Figure 3.14B, only the homogenate and P2 fraction is shown, as the differential affinity of $\alpha$-SAP97 was already determined in Figure 3.11. For the calculation of the levels of PSD-95 and PSD-93 $\alpha_{1 / 2}$ however, the data from the same blot was taken into account, which slightly differs from the values obtained in Figure 3.11B. PSD-93 $\alpha_{1 / 2}$ appears to be $20 \%$ of PSD-95 in homogenate, 
A

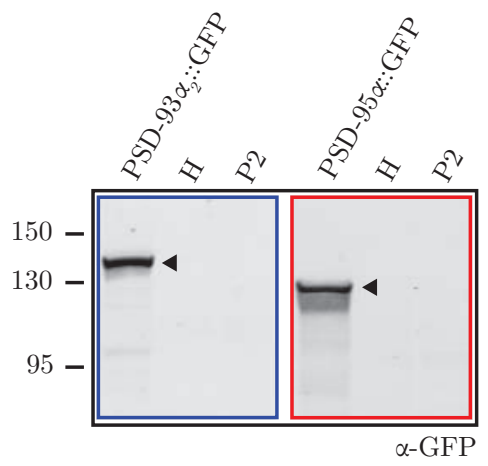

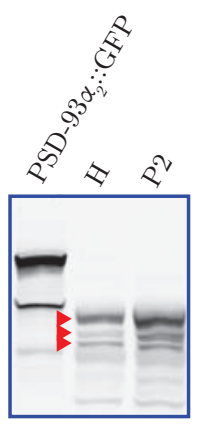

$\alpha-P S D-93$

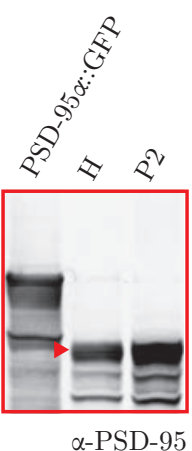

$\mathrm{B}$

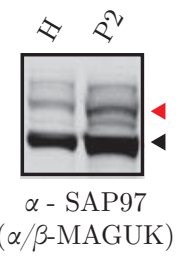

Figure 3.14: Quantification of PSD-93 and PSD-95 in the hippocampus. (A) PSD-93 $\alpha_{1}$ and PSD-95 $\alpha$ were overexpressed as GFP-fusion proteins in order to normalize the monoclonal $\alpha$-PSD-93 and $\alpha$-PSD-95 antibodies. The recombinant proteins are marked by black arrowheads, and the endogenous proteins are highlighted by red arrowheads. Having the information about their relative affinity, the amounts of PSD-93 and PSD-95 detected in hippocampal homogenate $(\mathrm{H})$ and $\mathrm{P} 2$ fraction could be quantitatively compared. In homogenate PSD-93 is about $42 \%$ of PSD-95 levels. PSD-93 enriches 2.2fold in the P2 fraction, whereas PSD-95 enriches 2.62-fold. (B) The same analysis as in (A) was performed using the $\alpha / \beta$-MAGUK antibody. The recombinant proteins for the normalization were loaded on the same western blot and probed simultaneously with $\alpha$ $\alpha / \beta$-MAGUK and $\alpha$-GFP. PSD-93 $\alpha_{1 / 2}$ appears to be $20 \%$ of the total PSD-95 levels in the homogenate, and $28 \%$ in the $\mathrm{P} 2$ fraction.

and reflects $28 \%$ in the $\mathrm{P} 2$ fraction.

Consistent with the band intensities of the PSD-93 isoforms in Figure 3.14A, PSD$93 \alpha_{1 / 2}$ thus accounts for $50 \%$ of all PSD-93 in the hippocampus. It is more enriched in the P2 fraction as the other isoforms. The lower two bands are each half as intense as PSD-93 $\alpha_{1 / 2}$.

\subsection{PSD-93 and PSD-95 interact in vivo}

It has previously been shown that recombinantly expressed PSD-93 $\alpha_{1}$ and PSD-95 $\alpha$ can form heteromultimers in COS-7 cells (Kim et al. 1996), but whether the other isoforms of PSD-93 can also interact with PSD-95 has not been studied. Also, it is not known whether PSD-93 and PSD-95 only bind when overexpressed in culture, or whether they 


\section{EXPRESSION OF PSD-93 IN THE HIPPOCAMPUS}

interact in vivo. N-terminal homomultimerization of PSD-95 $\alpha$ is essential for its effect on basal synaptic transmission (Xu et al. 2008), but whether the heteromultimerization with PSD-93 also occurs via the N-terminus has never been investigated.

I wanted to test whether a) PSD-93 and PSD-95 can interact in vivo, b) which of the PSD-93 isoforms is capable of interacting with PSD-95, and c) whether the interaction occurs via the N-terminal domain or via the C-terminus of PSD-95.

To answer the first question, I immunoprecipitated PSD-93 from hippocampal homogenate, which was solubilized with $1 \%$ sodium N-lauryl sarcosine. Because of the cross-reactivity of the monoclonal $\alpha$-PSD-93 antibody I performed the immunoprecipitation both with the monoclonal and the polyclonal antibody. I separated the isolated sample by SDS-PAGE, blotted the proteins onto a membrane and probed it first with monoclonal $\alpha$-PSD-93 and afterwards with $\alpha$-PSD-95. In Figure 3.15A, the PSD-93 bands appearing after the first antibody incubation are indicated by black arrows, whereas the PSD-95 band appearing after incubation with the $\alpha$-PSD-95 antibody is marked with a red arrow.

PSD-95 is co-immunoprecipitated with both the polyclonal and the monoclonal $\alpha$ PSD-93 antibody. However, the co-immunoprecipitation in the case of the monoclonal antibody is at least in part due to the cross-reactivity of the antibody since PSD-95 is also pulled down from homogenate of PSD-93 knockout mice (Fig. 3.15A, rightmost lane). The immunoprecipitation using the polyclonal $\alpha$-PSD-93 antibody suggests that the proteins interact in vivo.

In order to see which isoform of PSD-93 is capable of interacting with PSD-95, I overexpressed GFP-tagged recombinant PSD-93 in dissociated hippocampal cultures of rat. One week after infection, I solubilized the cells and immunoprecipitated with an $\alpha$-GFP (rb) antibody. Then I probed the western blot for a co-immunoprecipitation of endogenous PSD-95. As can be seen in Figure 3.15B, only PSD-93 $\alpha_{1}$ and PSD-93 $\alpha_{2}$ are able to pull down PSD-95. Thus, the interaction observed in vivo is likely to be via PSD-93 $\alpha_{1}$ and/or PSD-93 $\alpha_{2}$. Also, this result suggests that the interaction with PSD95 occurs via the immediate N-terminus of PSD-93, as the C-terminus of all PSD-93 isoforms is identical.

PSD-93 is not only capable of interacting with full-length PSD-95, but also with a truncated form of this protein which only contains the N-terminus and the first two PDZ-domains (Fig. 3.15C). Here, endogenous PSD-95 was replaced by GFP-tagged 
A

IP: $\alpha$-PSD-93 (pAb/mAb)

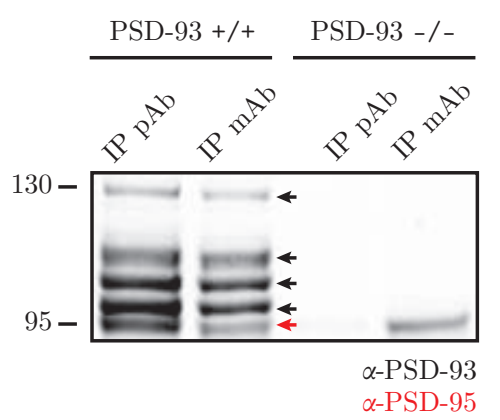

$\mathrm{B}$

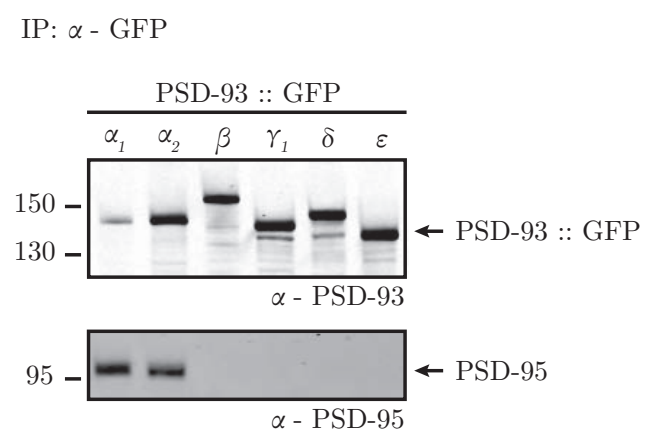

C

IP: $\alpha-$ GFP
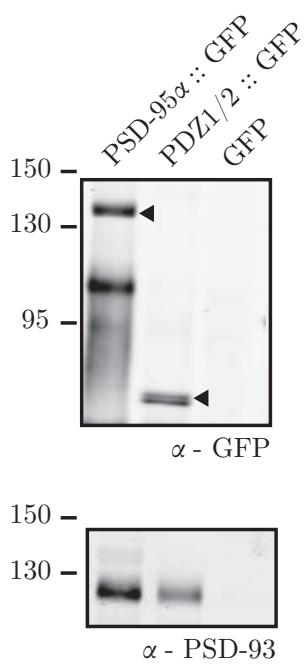

Figure 3.15: PSD-93 $\alpha_{1 / 2}$ and PSD-95 interact in vivo. (A) Immunoprecipitation of PSD-93 with the polyclonal (Lane 1,3) or monoclonal (Lane 2, 4) $\alpha$-PSD-93 antibody from WT (Lane 1,2) or PSD-93 -/- (Lane 3,4) mice. The western blot was probed with $\alpha$-PSD-93 and $\alpha$-PSD-95 monoclonal antibodies. (B) Only PSD-93 $\alpha_{1}$ and PSD-93 $\alpha_{2}$ can co-immunoprecipitate PSD-95 from dissociated hippocampal cultures. (C) PSD-93 can interact with the truncated PSD-95 (PDZ1/2). Overexpression GFP-tagged of full-length PSD-95 and truncated PSD-95 (N-terminus including PDZ1/2) in the absence of endogenous PSD-95. Immunoprecipitation with $\alpha$-GFP co-immunoprecipitates PSD-93. If only GFP is expressed, PSD-93 does not co-immunoprecipitate.

full-length or truncated PSD-95 in dissociated hippocampal cultures and immunoprecipitated with $\alpha$-GFP (rb). Endogenous PSD-93 can be detected on the western blot in case of both the full-length and truncated PSD-95, but not when only GFP is expressed (rightmost lane). This result shows that the interaction of PSD-93 $\alpha_{1 / 2}$ occurs with the N-terminus of PSD-95 $\alpha$, and not via the SH3 or GK domains.

In summary, PSD-95 and PSD-93 interact in vivo, and this interaction is most likely limited to the $\alpha$-isoforms of the two proteins. The data presented here indicates that the interaction occurs via the N-terminus of the two proteins, likely following the same 


\section{EXPRESSION OF PSD-93 IN THE HIPPOCAMPUS}

mechanism as in the homomerization of PSD-95 molecules.

With the use of antibodies I could show that PSD-93 $\beta$ and one or both of the $\alpha$ isoforms are expressed in the hippocampus. Also, I could demonstrate that PSD-93 $\delta$ makes up at most $5 \%$ of the band that runs at the height of PSD-93 $\alpha_{1 / 2}$. Figure 3.12 implies that PSD-93 $\gamma_{1}$ might be expressed in the hippocampus, but conclusive evidence is lacking, as this band could also be a truncation product of the $\alpha$-isoforms. N-terminal truncation has been previously described for PSD-95 $\alpha$ by Xu et al. (2008). It also

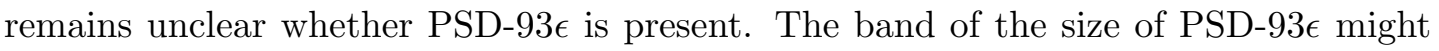
in fact be a truncation product of PSD-93 $\gamma_{1}$, as implicated in Figure 3.12.

\subsection{Analysis of PSD-93 by mass spectrometry}

Mass spectrometry (MS) is a sensitive analytical method to analyze the protein composition in a sample. In an MS experiment, proteins in a sample are digested into peptides by using an appropriate endoproteinase. Afterwards, the peptides are ionized because the mass spectrometric measurements are carried out in the gas phase. Ionization can be achieved by subjecting the peptides to a high electrical field (e.g. electrospray ionization, ESI). The ionized peptides enter the mass spectrometer, and their mass-to-charge $(\mathrm{m} / \mathrm{z})$ ratios are recorded together with their abundance.

In a tandem MS (MS/MS) experiment, the peptides are further analyzed to derive their amino acid sequence. This requires a special mass spectrometer, capable of selecting a single peptide precursor for further analysis. The chosen peptide is fragmented at its peptide backbone by collision with a gas (e.g. Argon, Xenon). By this, fragments of different lengths are generated, ranging from one amino acid to the full peptide. The $\mathrm{m} / \mathrm{z}$ spectrum of this mixture is generated. From the mass difference of the fragments, the amino acid sequence can be derived.

The MS and MS/MS spectra can be used to query a database which contains calculated masses from in silico digested proteins and fragmented peptides. Using an appropriate scoring algorithm (e.g. MOWSE, implemented in Mascot, Perkins et al. 1999), proteins matching the experimental data are retrieved.

In order to reduce the diversity of proteins in the sample, one can excise a band of an SDS-PAGE which contains the protein of interest. The digestion of the proteins 
can be performed "in-gel". Most commonly, the enzyme trypsin is used which cuts Cterminal of lysine $(\mathrm{K})$ or arginine $(\mathrm{R})$ residues, but usually not before proline $(\mathrm{P})$. Another enzyme commonly used to digest proteins for mass spectrometry is chymotrypsin. Chymotrypsin cuts C-terminal of tyrosine (Y), tryptophan (W), or phenylalanine (F), and leucine $(\mathrm{L})$ unless the next residue is a proline $(\mathrm{P})$. Unfortunately, it is possible that the digest is incomplete. Therefore, usually one or two missed cleavages are allowed for the in silico digested proteins.

In practice, some peptides are missed because they do not ionize well, are lost during sample preparation washing steps, or are too small to be detected by the mass spectrometer. In addition, long peptides pose a problem to the analysis. First, long peptides might be multiply-charged whereas in a standard MS experiment only doublyand triply-charged molecules are measured. Second, long peptides often do not fly well (e.g. because they are to acidic). Third, with increasing mass the resolution decreases, making it more difficult to match the experimental data with the in silico generated masses.

The following data result from a collaboration with Monika Raabe and Dr. Henning Urlaub (Bioanalytical Mass Spectroscopy Group, Max-Planck-Institute for Biophysical Chemistry, Göttingen, Germany).

\subsubsection{Identification of PSD-93 isoforms}

Because the PSD-93 isoforms differ at the N-terminus, I analyzed which peptides are theoretically generated by digestion with trypsin or chymotrypsin. In Table 3.2 all potential peptides generated by trypsin which are unique to a specific isoform are listed. Peptides smaller than six amino acids are excluded because they are unlikely to be detected by the mass spectrometer. For simplicity, missed cleavages are not listed, although peptides with up to two missed cleavages are included in the theoretical calculation of the $\mathrm{m} / \mathrm{z}$ ratios.

It becomes apparent that there are very few peptides unique for the $\alpha$-isoforms of PSD-93. For $\alpha_{1}$ there is only one N-terminal peptide, for $\alpha_{2}$ there are two, one being very short. Also, there are only three peptides theoretically available for PSD-93 $\gamma_{1}$, one for PSD-93 $\gamma_{2}$, and two for PSD-93є. In fact, T18, T19, and T28 might be too long to be efficiently analyzed. Only for PSD-93 $\beta$ and PSD-93 $\delta$ a variety of tryptic peptides 


\section{EXPRESSION OF PSD-93 IN THE HIPPOCAMPUS}

Table 3.2: N-terminal peptides generated by trypsin digest. Missed cleavages are ignored, as well as peptides shorter than 6 amino acids. Peptides in bold have been identified experimentally by MS/MS. Max Score is the maximum of the ions scores for the respective peptide as calculated by Mascot.

\begin{tabular}{|c|c|c|c|}
\hline Isoform & Unique peptide & ID & Max Score \\
\hline PSD-93 $\alpha_{1}$ & .MFFACYCALR.T & $\mathrm{T} 1$ & \\
\hline \multirow[t]{2}{*}{ PSD-93 $\alpha_{2}$} &. $\mathrm{MICHCK.V}$ & $\mathrm{T} 2$ & \\
\hline & K.VACTNNTLSLMFGCK.K & T3 & \\
\hline \multirow[t]{12}{*}{ PSD-93 $\beta$} & R.ALSLLEEYCK.K & $\mathrm{T} 4$ & 43 \\
\hline & R.KPEEQLLK.N & T5 & 26 \\
\hline & K.VMSIFK.S & T6 & \\
\hline & K.SSLFQALLDIQEFYEVTLLNSQK.S & $\mathrm{T} 7$ & \\
\hline & K.IEEANHVAQK.W & $\mathrm{T} 8$ & 39 \\
\hline & K.TLLLDSCR.D & T9 & 35 \\
\hline & K.SSEHASCSGPK.E & $\mathrm{T} 10$ & 15 \\
\hline & K.ENALYIEQNK.E & $\mathrm{T} 11$ & \\
\hline & K.ENQCSENETEEK.T & $\mathrm{T} 12$ & \\
\hline & K.TCQNQGK.C & $\mathrm{T} 13$ & \\
\hline & K.CPAQNCSVEAPTWMPVHHCTK.Y & $\mathrm{T} 14$ & \\
\hline & K.VNGTEIEYEFEEITLER.G & $\mathrm{T} 15$ & \\
\hline \multirow[t]{3}{*}{ PSD-93 $\gamma_{1}$} & .MLPTFDMQRPSASR.A & $\mathrm{T} 16$ & \\
\hline & R.AENYQLLWDTIASLK.Q & $\mathrm{T} 17$ & 62 \\
\hline & K.QCEEAMQHAFIPASPAPIIVNTDTLDTIPYVNGTEIEYEFEEITLER.G & $\mathrm{T} 18$ & \\
\hline PSD-93 $\gamma_{2}$ & .MQHAFIPASPAPIIVNTDTLDTIPYVNGTEIEYEFEEITLER.G & T19 & \\
\hline \multirow[t]{9}{*}{ PSD-93 } & .MNAYLTK.Q & $\mathrm{T} 20$ & \\
\hline & K.QHSCSR.G & $\mathrm{T} 21$ & \\
\hline & R.GSDGMDIGR.S & $\mathrm{T} 22$ & \\
\hline & R.SAPTLIR.D & $\mathrm{T} 23$ & 17 \\
\hline & R.DAHCACGWQR.N & $\mathrm{T} 24$ & \\
\hline & R.NAQGLGYSSQTMPSSGPGGPASNR.T & $\mathrm{T} 25$ & \\
\hline & K.LVTLWDSVR.K & $\mathrm{T} 26$ & 62 \\
\hline & K.GNCGER.C & $\mathrm{T} 27$ & \\
\hline & R.CACPHGWFSPAQASPAPIIVNTDTLDTIPYVNGTEIEYEFEEITLER.G & $\mathrm{T} 28$ & \\
\hline \multirow[t]{2}{*}{ 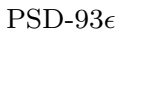 } & .MFASIWYAK.K & T29 & 5 \\
\hline & R.FVHNAR.K & T30 & \\
\hline Isoforms & Peptide unique for subset & ID & Max Score \\
\hline \multirow{4}{*}{$\begin{array}{l}\text { PSD-93 } \alpha_{1} / \\
\alpha_{2}, \beta\end{array}$} & R.YQDEDGPHDHSLPR.L & T31 & 72 \\
\hline & R.LTHEVR.G & T32 & 32 \\
\hline & R.GPELVHVSEK.N & T33 & 37 \\
\hline & K.NLSQIENVHGYVLQSHISPLK.(AV) & T34 & 35 \\
\hline $\begin{array}{l}\text { PSD-93 } \alpha_{1} / \\
\alpha_{2}, \epsilon\end{array}$ & K.ASPAPIIVNTDTLDTIPYVNGTEIEYEFEEITLER.G & T35 & 50 \\
\hline
\end{tabular}


is available. This is promising for finding matches in case the isoforms are present in the sample.

In two separate experiments, PSD-93 was immunoprecipitated from hippocampal tissue, subjected to SDS-PAGE and the gel band corresponding to the size of PSD93 excised (95-130kDa). The proteins were digested "in-gel", and separately analyzed with tandem MS. Peptides that were identified and are unique for a single isoform are shown in bold in Table 3.2.

No peptides were found that are unique for PSD-93 $\alpha_{1}$ or PSD-93 $\alpha_{2}$. Five peptides unique for PSD-93 $\beta$ (T4, T5, T8, T9 and T10) were detected, which all have a high ions score and are predicted by several hits. One peptide for PSD-93 $\gamma_{1}$ was found (T17) which also has a high ions score. Interestingly, this peptide is specific for the long isoform of PSD-93 $\gamma$. Still, it is possible that the short version as predicted by Brenman et al. (1996) and Parker et al. (2004) is additionally expressed in the hippocampus. However, although translation initiation might happen at the second start codon, bicistronic eukaryotic mRNAs are infrequent (for a review on translation initiation see Gray and Wickens 1998). PSD-93 $\delta$ is also identified in the sample and the peptides T23 and T26 have high scores. PSD-93 $\epsilon$ is predicted by one peptide, which has a very low score and which was not found in the second experiment. Therefore, it is not sure whether this is a false positive hit or not. Either the two peptides unique for PSD-93 $\epsilon$ are not ionized well, or the protein is only present in the hippocampus in traces, if at all. One peptide, T35, occurs with a high ions score and is predictive for PSD-93 $\alpha_{1}$, PSD-93 $\alpha_{2}$, and/or PSD-93€.

A similar analysis was done using the enzyme chymotrypsin to digest the protein into a different set of peptides. By this I hoped to observe masses specific for PSD-93 $\alpha_{1}$ and/or PSD-93 $\alpha_{2}$.

The number of unique chymotryptic peptides for each isoform is lower than with the digestion with trypsin (see Table 3.3). Still, for PSD-93 $\alpha_{1}$ and PSD-93 $\alpha_{2}$ there are again one or two unique peptides theoretically available. However, none of them is observed experimentally. For PSD-93 $\beta$ and PSD-93 $\delta$ several peptides are possible, but only one of each isoform is observed, and only with a small score compared to the one observed with the tryptic peptides. Importantly, there is one peptide, C23, which is unique for the $\alpha$-isoforms of PSD-93. Although the ions score is only 19 and thus 


\section{EXPRESSION OF PSD-93 IN THE HIPPOCAMPUS}

Table 3.3: N-terminal peptides generated by chymotrypsin digest. Missed cleavages are ignored, as well as peptides shorter than 6 amino acids. Peptides in bold have been identified by MS/MS. Max Score is the maximum of the ions scores for the respective peptide as calculated by Mascot.

\begin{tabular}{|c|c|c|c|}
\hline Isoform & Unique peptide & ID & Max Score \\
\hline PSD-93 $\alpha_{1}$ & L.RTNVKKY.R & $\mathrm{C} 1$ & \\
\hline \multirow[t]{2}{*}{ PSD-93 $\alpha_{2}$} & .MICHCKVACTNNTL.S & $\mathrm{C} 2$ & \\
\hline & F.GCKKY.R & $\mathrm{C} 3$ & \\
\hline \multirow[t]{9}{*}{ PSD-93 $\beta$} & .MPVKKKDTDRAL.S & $\mathrm{C} 4$ & \\
\hline & L.RKPEEQL.L & $\mathrm{C} 5$ & \\
\hline & L.KNAVKKVMSIF.K & $\mathrm{C} 6$ & \\
\hline & L.NSQKSCEQKIEEANHVAQKW.E & $\mathrm{C} 7$ & \\
\hline & L.DSCRDSL.Q & $\mathrm{C} 8$ & \\
\hline & L.QKSSEHASCSGPKENAL.Y & C9 & 4 \\
\hline & Y.IEQNKENQCSENETEEKTCQNQGKCPAQNCSVEAPTW.M & $\mathrm{C} 10$ & \\
\hline & W.MPVHHCTKY.R & $\mathrm{C} 11$ & \\
\hline & L.KVNGTEIEY.E & $\mathrm{C} 12$ & \\
\hline \multirow[t]{3}{*}{ PSD-93 $\gamma_{1}$} & F.DMQRPSASRAENY.Q & $\mathrm{C} 13$ & \\
\hline & W.DTIASL.K & $\mathrm{C} 14$ & \\
\hline & L.KQCEEAMQHAF.I & $\mathrm{C} 15$ & \\
\hline PSD-93 $\gamma_{2}$ & - & & \\
\hline \multirow[t]{6}{*}{ PSD-93 $\delta$} & L.TKQHSCSRGSDGMDIGRSAPTL.I & $\mathrm{C} 16$ & \\
\hline & L.IRDAHCACGW.Q & $\mathrm{C} 17$ & \\
\hline & W.QRNAQGL.G & $\mathrm{C} 18$ & 14 \\
\hline & Y.SSQTMPSSGPGGPASNRTKL.V & C19 & \\
\hline & W.DSVRKSPHKTSTKGKGNCGERCACPHGW.F & $\mathrm{C} 20$ & \\
\hline & F.SPAQASPAPIIVNTDTL.D & $\mathrm{C} 21$ & \\
\hline 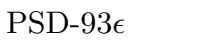 & F.VHNARKAKSEKASPAPIIVNTDTL.D & $\mathrm{C} 22$ & \\
\hline Isoforms & Peptide unique for subset & ID & Max Score \\
\hline PSD-93 $\alpha_{1}, \alpha_{2}$ & L.KASPAPIIVNTDTLDTIPY.V & $\mathrm{C} 23$ & 19 \\
\hline
\end{tabular}


lower than the threshold of 20, the MS/MS mass spectrum resembles the amino acid sequence well and hence this peptide likely reflects a true hit.

Both for the digestion with trypsin and chymotrypsin the very N-terminal peptides for $\alpha_{1}$ and $\alpha_{2}$ are not experimentally detected. The $\alpha$-isoforms have been shown to be palmitoylated (El-Husseini et al. 2000c). Addition of palmitate acid of course changes the mass of the peptide $(+238 \mathrm{Da}$ for each palmitate acid). Therefore, we manually calculated the mass to be observed for the respective peptide if one or two palmitate acids are present. The mass spectra were searched for the calculated masses, but no hit was found. In general, palmitoylated peptides can be efficiently ionized and identified by mass spectrometry (Hoffman and Kast 2006; Papac et al. 1992). Still, these specific N-terminal peptides might just not be ionized well, independent of the fact that they are palmitoylated.

The mass spectrometry results support my previous results that one or both $\alpha$ isoform of PSD-93 are expressed in the hippocampus, and that PSD-93 $\beta$ is present. It goes along with the results of using the PSD- $93 \delta$-antiserum, that this isoform is also expressed in the hippocampus. A novel finding is that the long $\gamma$-isoform, PSD-93 $\gamma_{1}$, is expressed. Whether PSD-93€ is present in or absent from the hippocampus cannot be unequivocally deduced.

\subsubsection{Possible interaction partners of PSD-93}

In addition to identifying PSD-93 isoforms expressed in the hippocampus, mass spectrometry also allows to identify possible interaction partners of PSD-93. In the band containing PSD-93, a lot of other proteins were found. In order to remove unspecific proteins, the immunoprecipitation of PSD-93 was simultaneously performed in WT and PSD-93 KO animals.

The samples were processed in parallel, and the band shown in Figure 3.16 was excised for analysis. This band contains proteins that co-immunoprecipitated specifically or unspecifically with the PSD-93 antibody or the anti-mouse IgG-agarose. Only proteins of size of approximately $95-135 \mathrm{kDa}$ are considered, as the band of this weight range was excised.

The MS and MS/MS spectra were matched with the refseq_mouse database, and identified proteins with a protein score higher or equal to 50 were considered for the 


\section{EXPRESSION OF PSD-93 IN THE HIPPOCAMPUS}

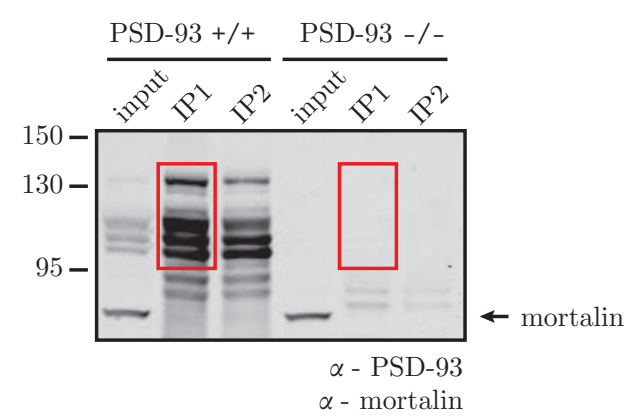

Figure 3.16: Immunoprecipitation of PSD-93 from hippocampal tissue of PSD$93 \mathrm{WT}$ and KO mice. In lane 1 and 4 the input is shown, from which PSD-93 was immunoprecipitated. In the case of the KO mice, PSD-93 is absent. The lowest band shows mortalin, a mitochondrial stress protein used to demonstrate equal amounts of input for WT and KO. In lanes 2, 3, 5, and 6, the immunoprecipitated protein sample is loaded. One can see a strong PSD-93 signal in case of the WT and no signal in case of the KO mice.

analysis. This threshold is a bit lower and hence less conservative than the calculated threshold of 59 (see p.31). The proteins from trypsin and chymotrypsin digested samples were combined and redundant entries as well as obvious contaminations such as keratin removed. A total of 195 proteins were isolated from WT animals and 106 proteins from PSD-93 KO mice. Of these proteins, 37 were found in both groups, indicating unspecific proteins. The remaining 69 proteins exclusively found in the KO sample most likely result from the cross-reactivity of the monoclonal $\alpha$-PSD-93 antibody with PSD-95, as shown in Figure 3.7B. In the absence of PSD-93, the binding of this antibody to PSD-95 might even be enhanced. 158 proteins were exclusively found in the PSD-93 WT mice. These proteins were classified into functional groups with the help of the UniProt database (UniProt Consortium 2010).

Of the proteins directly involved in synaptic transmission, the glutamate receptor subunits NR1 and GluR2 were identified. It is not surprising that NR2A and NR2B were not detected, although they have been shown to directly bind to PSD-MAGUK proteins. With their molecular weight of $180 \mathrm{kDa}$ they were too big to be included in the excised band. Also, there is no experimental data showing direct interaction of PSD-93 and GluR2. Instead, the interaction occurs via TARPs that are too small ( $\sim 36 \mathrm{kDa})$ to be present in the excised band. 


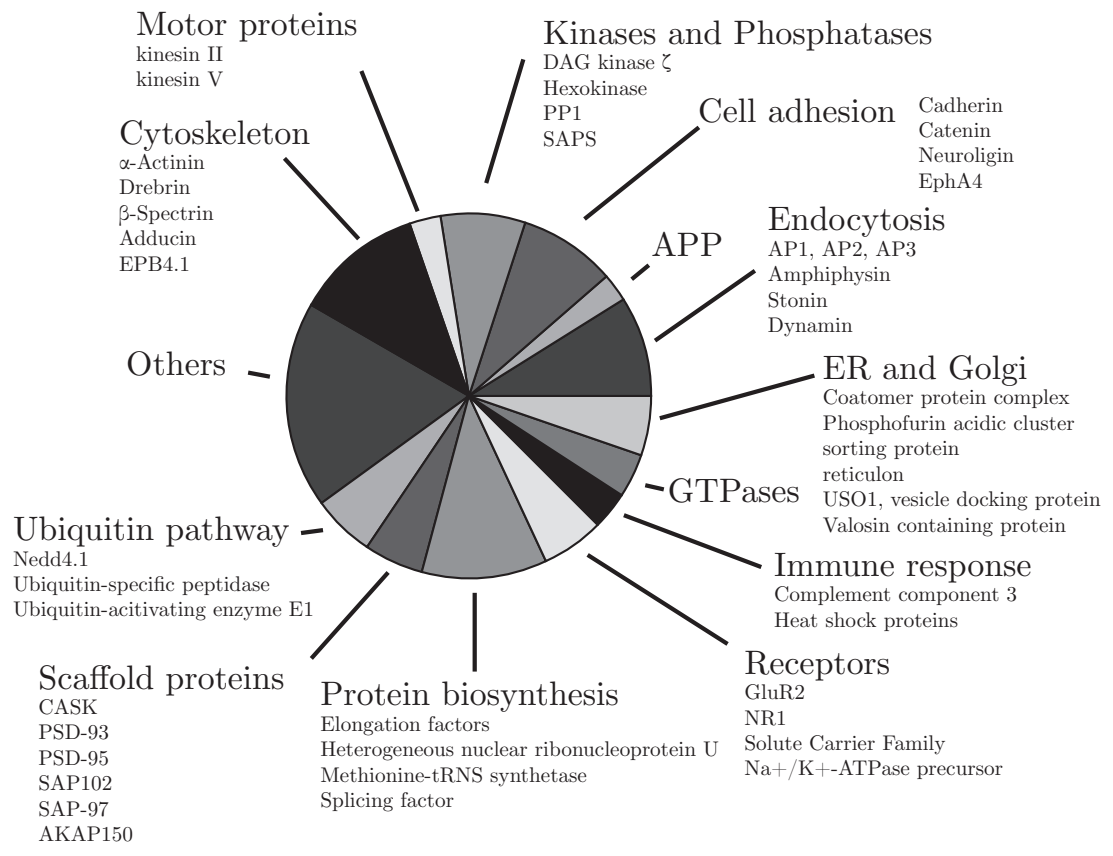

Figure 3.17: Classification of proteins that co-immunoprecipitated with PSD-

93. Proteins co-immunoprecipitated with PSD-93 are classified into cytoskeleton proteins $(12 \%)$, motor proteins $(3 \%)$, kinases and phosphatases (8\%), cell adhesion (8\%), APP (3\%), endocytosis (9\%), ER/Golgi (5\%), GTPases and regulators (4\%), immune response $(3 \%)$, ion transport and receptors $(6 \%)$, protein biosynthesis and RNA processing (11\%), scaffold proteins (5\%), ubiquitin conjugation pathway (6\%), and others (18\%).

Apart from PSD-95, also the other PSD-MAGUKs, SAP97 and SAP102 were found to co-immunoprecipitate. So far, it has not been shown that SAP102 interacts with the other PSD-MAGUKs. Observing SAP102 likely results from an indirect association via the NMDA receptor complex which binds multiple PSD-MAGUK proteins. CASK and AKAP150, members of the PSD-protein scaffold, were also identified in the coimmunoprecipitate.

Several proteins involved in the actin cytoskeleton, such as actinin, were found to coimmunoprecipitate with PSD-93. Motor proteins of the kinesin family and microtubuleassociated proteins were found. Also, known interactors of the cell-cell adhesion class, e.g. neuroligin, were detected. In addition, four isoforms of the Amyloid precursor protein (APP) were immunoprecipitated. APP is expressed in many tissues and as an integral membrane protein it is enriched in the synapses of neurons. The exact function 


\section{EXPRESSION OF PSD-93 IN THE HIPPOCAMPUS}

of APP is still oblivious, but it has been suggested to play a role in synapse formation and repair. Also, it might be important as a cell adhesion molecule and in cell signaling.

Proteins of the AP2 adaptor complex, that mediate endocytosis of clathrin-coated vesicles, and the GTPase dynamin, which is required to pinch off the clathrin-coated invaginations to produce intracellular vesicles, were found. It has been suggested that the AP2 adaptor complex and PSD-95 bind to adjacent regions of the C-terminus of NR2B (Lavezzari et al. 2003), and that AP2 binding negatively regulates PSD-95 binding (Roche and Huganir 2001). The binding of AP2 and NR2B is also regulated by phosphorylation by Fyn (Prybylowski et al. 2005), which is mediated by an interaction of this kinase with PSD-93 (Nada et al. 2003; Sato et al. 2008).

Several proteins of the ubiquitin conjugation pathway were identified. Three isoforms of the E3 ubiquitin ligase Nedd4-1 were observed, a protein which has been suggested to interact with SAP102 (Kawabe et al. 2010). It remains to be seen whether this interaction is only via SAP102 or whether PSD-93 might be ubiquitinated by Nedd4-1 as well. 


\section{The role of PSD-93 in basal synaptic transmission}

Neurons are specialized in generating electrical signals in form of action potentials and propagating them across synapses to other neurons. Changes in the strength of these connections can be analyzed in a very sensitive manner with electrophysiological techniques. The PSD-MAGUKs have been implicated to play a role in regulating synaptic strength by affecting AMPA receptor trafficking and function. The role of PSD-93, however, remains largely elusive. In the following sections, the results of knockdown of PSD-93, overexpression of single isoforms and chimeric constructs as well as replacement of PSD-95 with PSD-93 isoforms are shown.

A typical experimental setup is illustrated in Figure 4.1. In all experiments, hippocampal slice cultures of P7-P9 Wistar rats were prepared, infected at DIV1-2 with lentiviral particles and recordings were performed 4-9 days post-infection (Fig. 4.1B). No difference was observed with respect to the day of infection (DIV1 or DIV2), the duration of infection, or the age of the rats used. In most of the experiments presented here, an infected cell and an uninfected neighboring cell (control) are simultaneously voltage-clamped at $-60 \mathrm{mV}$ or at $+40 \mathrm{mV}$ and the synaptic responses to a common input are compared (Fig. 4.1C). NMDAR EPSCs were approximated by recording at $+40 \mathrm{mV}, 60 \mathrm{msec}$ after the peak, when AMPAR-mediated currents have largely decayed as can be seen by recording in the presence of the NMDAR antagonist AP-V $(50 \mu \mathrm{M})$ in Fig. 4.1C. 
A

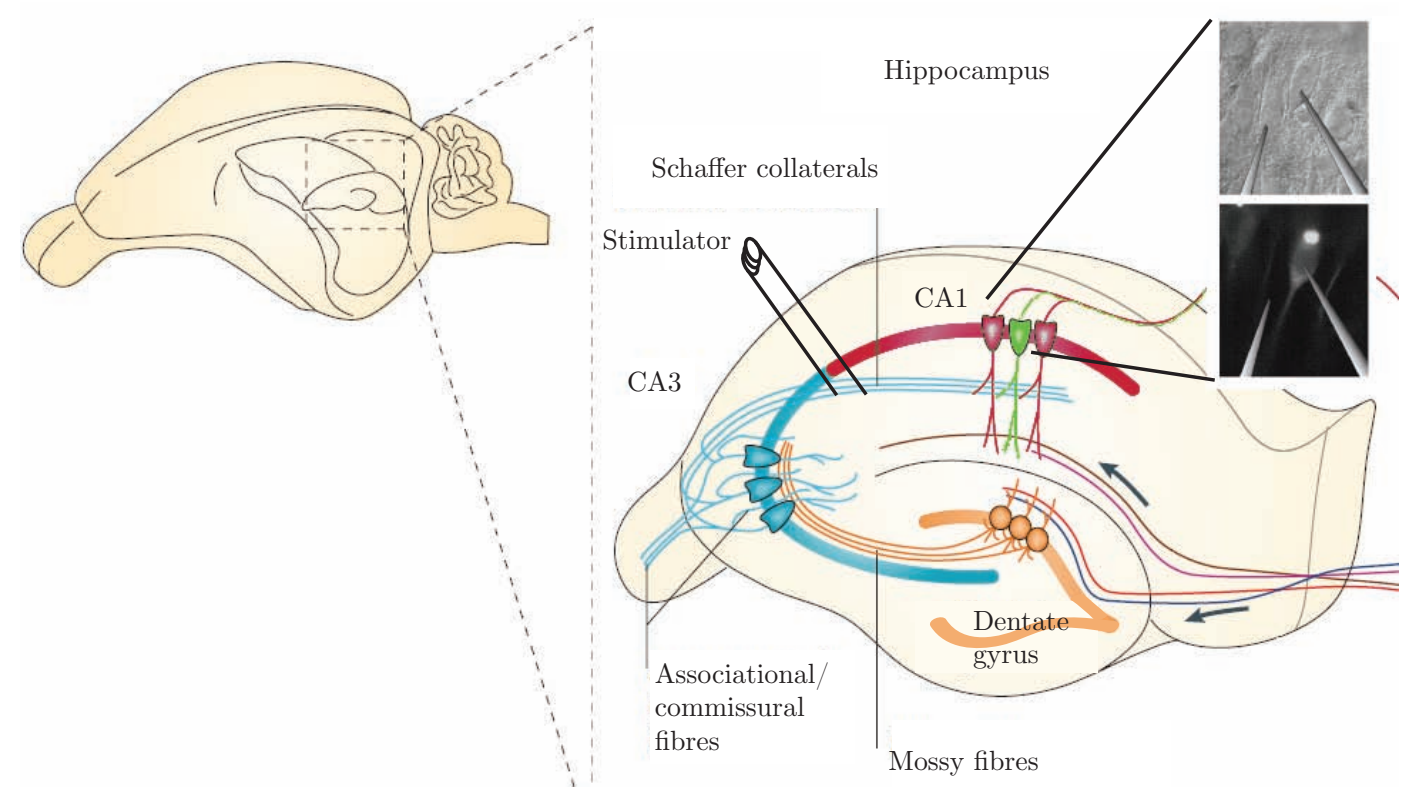

$\mathrm{B}$

C
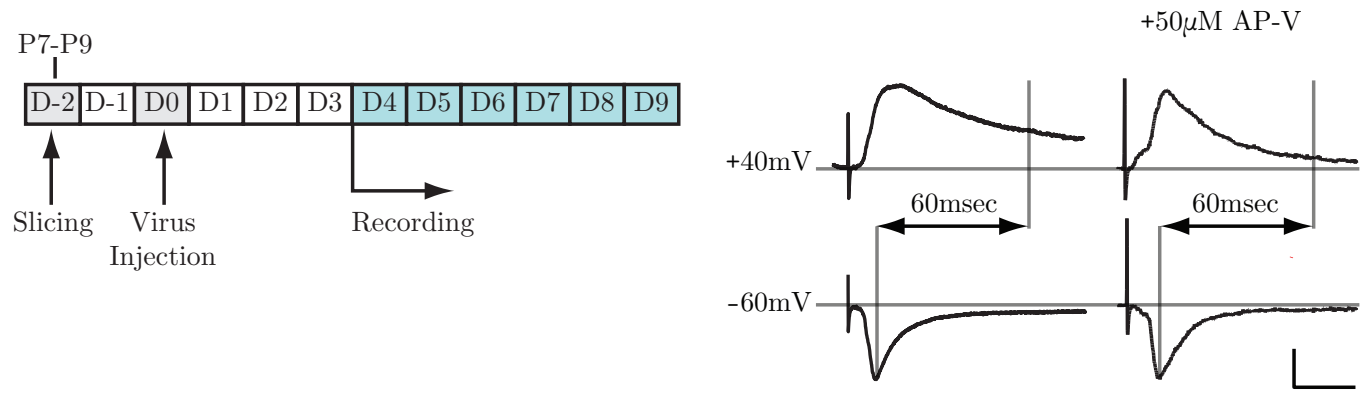

Figure 4.1: Simultaneous dual whole-cell patch-clamp recordings of hippocampal slice cultures. (A) Scheme of the anatomical location of the hippocampus and a cross section showing the hippocampal formation. Lentivirus was used to infect single cells in the CA1 region (indicated in green) which could be visualized with fluorescent light sources (inset). Recordings were performed by voltage-clamping an infected and a neighboring control cell while stimulating the Schaffer collateral pathway (Figure adapted from Neves et al. 2008). (B) Time scale of the experiment. Slices were prepared from P7 to P9 Wistar rats and infected at DIV2. The recordings were performed from DIV6 on. (C) The cells were voltage-clamped at $-60 \mathrm{mV}$ to record AMPAR EPSCs. NMDAR EPSCs were approximated by recording at $+40 \mathrm{mV}, 60 \mathrm{msec}$ after the peak, when AMPAR-mediated currents have largely decayed as can be seen by recording in the presence of $50 \mu \mathrm{M} \mathrm{AP-V}$. Scale bars 25pA, $25 \mathrm{msec}$. 


\subsection{Knockdown of PSD-93 does not affect basal synaptic transmission}

The literature on the physiological effect of the loss of PSD-93 is scarce, and its function in synaptic transmission remains puzzling. Synaptic physiology of cerebellar Purkinje neurons and basal synaptic transmission in the hippocampus was normal in the PSD93 KOs (Carlisle et al. 2008; Elias et al. 2006; McGee et al. 2001), and it has been suggested that other members of the PSD-MAGUK family can compensate for the loss of PSD-93. One previous study has postulated that RNAi-mediated acute knockdown of PSD-93 in the hippocampus shows the same phenotype as acute loss of PSD-95, namely a $50 \%$ reduction of AMPAR EPSCs (Elias et al. 2006).

In contrast to Elias et al. (2006), in my hands none of the three constructs that successfully reduce the protein levels of PSD-93 (Fig. 3.6) affect basal synaptic transmission. Both AMPAR- and NMDAR-mediated EPSCs of the infected neurons are not significantly different from the control cells (Fig. 4.2). In these scatter plots, each gray circle represents the averaged amplitudes of infected and control cell of a single experiment. The black circle shows mean \pm SEM. Two of the constructs, namely rn931 and rn932, have been used in a previous study and resulted in the above mentioned $50 \%$ reduction of AMPAR EPSCs (Elias et al. 2006). Here, rn931 and rn932 as well as sh93d do not change the amplitude of AMPAR or NMDAR EPSCs.

Apparently, PSD-95 and PSD-93 behave differently with respect to basal synaptic transmission. Whereas acute loss of PSD-95 decreases AMPAR EPSCs by 50\% (see also Fig. 4.9A), acute loss of PSD-93 has no effect on basal synaptic transmission.

\subsection{Overexpression of PSD-93 isoforms shows differential effects}

In order to delineate the functional difference of the PSD-93 isoforms, I used a gainof-function approach by overexpressing the individual isoforms in hippocampal slice cultures of WT animals. In previous studies, only the effect of overexpressing PSD-93 $\alpha_{1}$ has been studied, which resulted in a three-fold increase in AMPAR EPSCs (Elias et al. 2006). No comparative characterization of the individual isoforms has been performed with respect to basal synaptic transmission or plasticity. 
A $\operatorname{sh} 93 d$
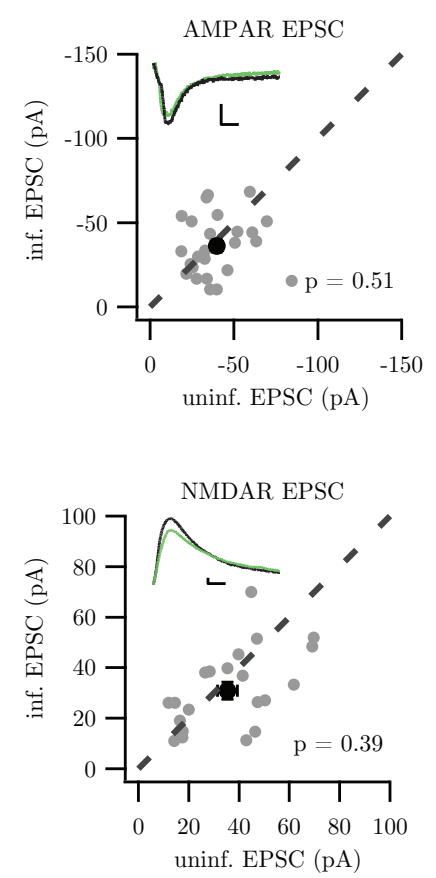

B rn931
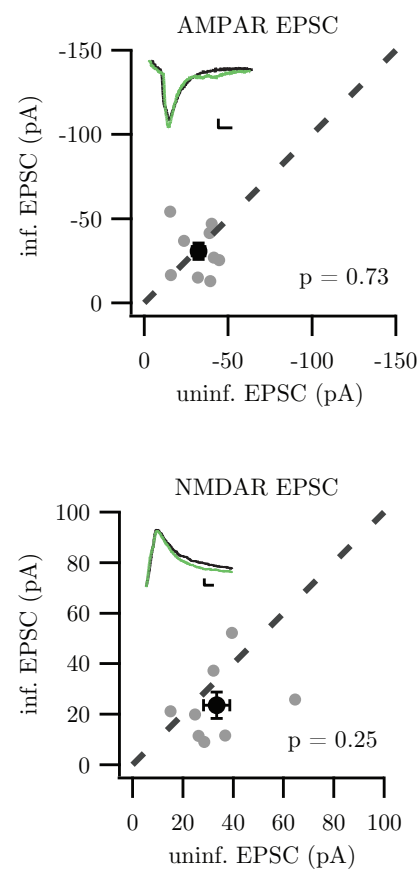

C $\operatorname{rn} 932$
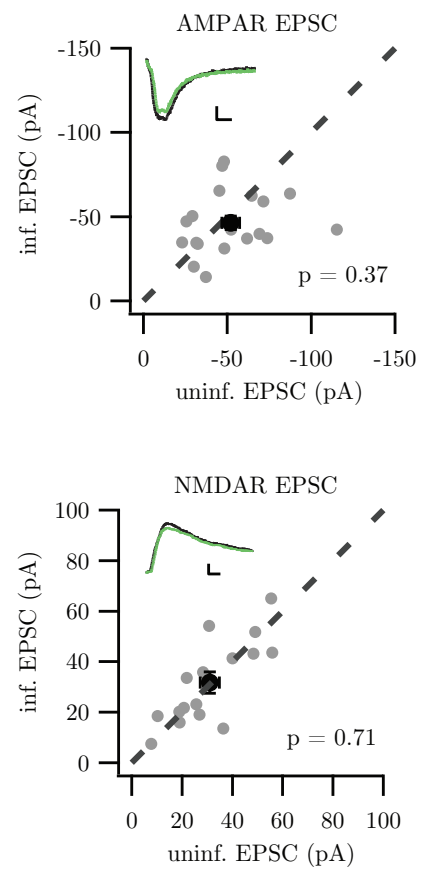

Figure 4.2: Knockdown of PSD-93 with shRNAs does not affect AMPAR or NMDAR EPSCs. (A) Amplitudes of AMPAR EPSCs (top; control: $-39.8 \pm 3.4 \mathrm{pA}$, infected: $-36.2 \pm 3.5 \mathrm{pA} ; \mathrm{N}=25$ ) and NMDAR EPSCs (bottom; control: $35.5 \pm 3.9 \mathrm{pA}$, infected: $30.9 \pm 3.4 \mathrm{pA} ; \mathrm{N}=22$ ) in cells expressing sh93d versus neighboring uninfected controls. Sample traces of infected (green) and control cell (black). (B) Amplitudes of AMPAR EPSCs (top; control: $-32.3 \pm 3.8 \mathrm{pA}$, infected: $-30.7 \pm 5.0 \mathrm{pA} ; \mathrm{N}=9$ ) and NMDAR EPSCs (bottom; control: $33.4 \pm 5.2 \mathrm{pA}$, infected: $23.5 \pm 5.2 \mathrm{pA} ; \mathrm{N}=8$ ) in cells expressing rn931 versus uninfected control cells. Sample traces of infected (green) and control cell (black). (C) Amplitudes of AMPAR EPSCs (top; control: $-52.1 \pm 5.2 \mathrm{pA}$, infected: $-46.2 \pm 4.0 \mathrm{pA} ; \mathrm{N}=20$ ) and NMDAR EPSCs (bottom; control: $31.0 \pm 3.8 \mathrm{pA}$, infected: $31.8 \pm 4.2 \mathrm{pA} ; \mathrm{N}=16$ ) in cells expressing rn932 versus uninfected controls. Sample traces of infected (green) and control cell (black). Scale bars 10pA, 10msec. 
Surprisingly, none of the $\alpha$-isoforms of PSD-93 led to a change in AMPAR EPSCs (PSD-93 $\alpha_{1}$ control: $-48.6 \pm 4.0 \mathrm{pA}$, infected: $-43.7 \pm 6.2 \mathrm{pA}$; PSD-93 $\alpha_{2}$ control: -65.9 $\pm 9.5 \mathrm{pA}$, infected: $-58.7 \pm 5.3 \mathrm{pA}$ ) or NMDAR EPSCs (PSD-93 $\alpha_{1}$ control: $17.7 \pm 2.6 \mathrm{pA}$, infected: $18.7 \pm 2.9 \mathrm{pA}$; PSD-93 $\alpha_{2}$ control: $35.5 \pm 5.1 \mathrm{pA}$, infected $41.2 \pm 7.4 \mathrm{pA}$ ). The data is illustrated in Figure 4.3A and 4.3B.

\section{A PSD- $93 \alpha_{1}$}
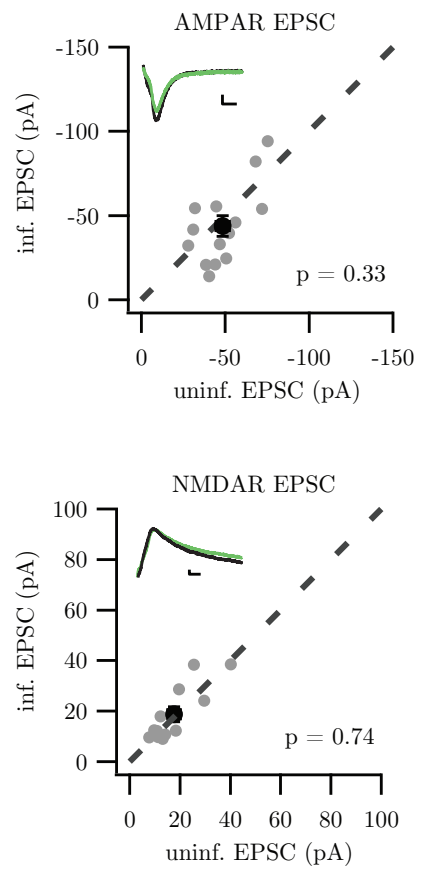

B PSD-93 $\alpha_{2}$
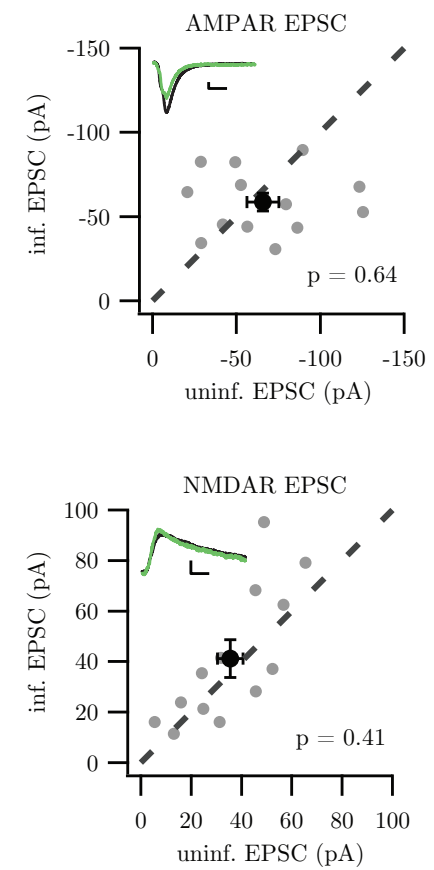

C PSD-93 $\alpha_{2}$ IRES-GFP
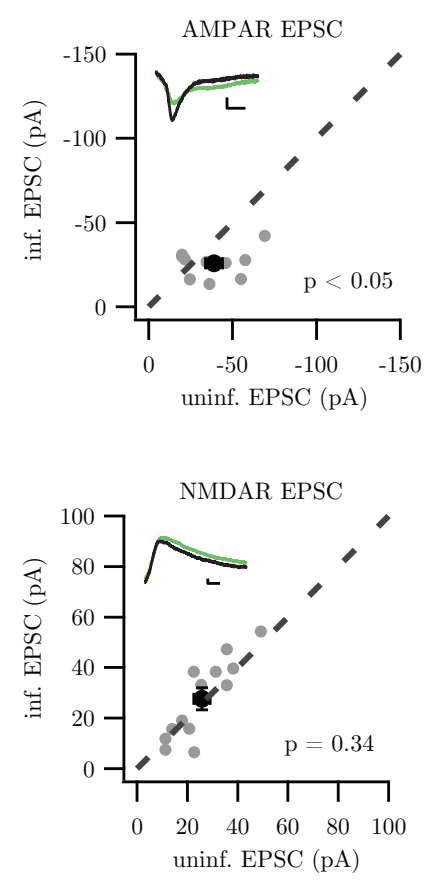

Figure 4.3: Overexpressing the $\alpha$-isoforms of PSD-93 does not increase AMPAR EPSCs. (A) Amplitudes of AMPAR EPSCs (top, N=14) and NMDAR EPSCs (bottom, N=13) in cells expressing GFP-tagged PSD-93 $\alpha_{1}$ versus uninfected controls. Sample traces of infected (green) and control cell (black). (B) Amplitudes of AMPAR EPSCs (top, N=13) and NMDAR EPSCs (bottom, N=13) in cells expressing GFP-tagged PSD-93 $\alpha_{2}$ versus uninfected controls. Sample traces of infected (green) and control cell (black). (C) Amplitudes of AMPAR EPSCs (top, N=11) and NMDAR EPSCs (bottom, $\mathrm{N}=13$ ) in cells expressing untagged PSD-93 $\alpha_{2}$ versus uninfected control. Sample traces of infected (green) and control cell (black). Scale bars 10pA, 10msec.

In order to rule out that the GFP-tag impairs the proper folding of the protein and renders it non-functional, PSD-93 $\alpha_{2}$ was also overexpressed as a bicistronic construct, separating PSD-93 $\alpha_{2}$ and GFP expression with an IRES cassette. Still, no 


\section{THE ROLE OF PSD-93 IN BASAL SYNAPTIC TRANSMISSION}

increase of AMPAR EPSC amplitude was observed. Instead, there was even a reverse effect in that AMPAR EPSCs were slightly reduced in the infected cells as shown in Figure 4.3C (control: $-38.89 \pm 5.08 \mathrm{pA}$, infected: $-25.80 \pm 2.43 \mathrm{pA}, \mathrm{p}=0.04$ ). NMDAR EPSCs, however, were not altered (control: $25.8 \pm 3.2 \mathrm{pA}$, infected: $27.7 \pm 4.4 \mathrm{pA}, \mathrm{p}=0.34$ ).

The isoform PSD-93 $\beta$, which contains an L27 domain at the N-terminus similar to PSD-95 $\beta$ and SAP97 $\beta$, showed no effect on AMPAR or NMDAR EPSCs (Fig. 4.4A; AMPAR EPSCs control: $-44.6 \pm 5.7 \mathrm{pA}$, infected: $-46.4 \pm 4.9 \mathrm{pA}$; NMDAR EPSCs control: $37.6 \pm 4.4 \mathrm{pA}$, infected: $35.7 \pm 4.0 \mathrm{pA}$ ). This was expected from a bona fide $\beta$-isoform, as overexpressing PSD-95 $\beta$ and SAP97 $\beta$ also did not affect basal synaptic transmission (Schlüter et al. 2006).

Neurons infected to overexpress PSD-93 $\gamma_{1}$ also did not show any change in basal synaptic transmission (Fig. 4.4B). Both AMPAR and NMDAR EPSCs did not differ significantly from each other in the infected cell compared to the control neuron (AMPAR EPSCs control: $-33.4 \pm 2.2 \mathrm{pA}$, infected: $-42.0 \pm 7.1 \mathrm{pA}$; NMDAR EPSCs control: $28.7 \pm 3.5 \mathrm{pA}$, infected: $36.1 \pm 7.1 \mathrm{pA})$. PSD-93 $\gamma_{2}$ was not further characterized as the construct which drives the expression of PSD-93 $\gamma_{1}$ contains the start codon for PSD-93 $\gamma_{2}$. Thus, potentially, the short isoform could also be translated. However as discussed in Section 3.5.1, most likely the $\gamma$-isoform only occurs in the long $\left(\gamma_{1}\right)$ version.

Overexpression of PSD-93 significantly enhanced AMPAR EPSCs (Figure 4.4C; control: $-37.4 \pm 4.1 \mathrm{pA}$, infected: $-53.5 \pm 4.9 \mathrm{pA})$. In addition to this $43 \%$ increase of

Figure 4.4 (facing page): Overexpressing the non- $\alpha$-isoforms of PSD-93 has differential effects on basal synaptic transmission. (A) Scatter plot of amplitudes of cells expressing PSD-93 $\beta$ and neighboring control neurons (top: AMPAR EPSCs, N=14; bottom: NMDAR EPSCs, N=16). Sample traces of infected (green) and control cell (black). (B) Scatter plot of AMPAR EPSCs (top, N=9) and NMDAR EPSCs (bottom, $\mathrm{N}=9$ ) of cells expressing PSD-93 $\gamma_{1}$ and neighboring control cells. Sample traces of infected (green) and control cell (black). (C) Amplitudes of AMPAR EPSCs (top, N=18) and NMDAR EPSCs (bottom, N=18) of neurons expressing PSD-93 versus control cells. Sample traces of infected (green) and control cell (black). Sample traces of infected (green)

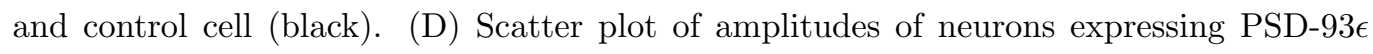
versus control neurons (top: AMPAR EPSCs, N=11; bottom: NMDAR EPSCs, N=10). Sample traces of infected (green) and control cell (black). (E) Summary graph showing the ratio of infected and control cell for AMPAR EPSCs (top) and NMDAR EPSCs (bottom). ${ }^{*} \mathrm{p}<0.05,{ }^{* * *} \mathrm{p}<0.001$. Scale bars $10 \mathrm{pA}, 10 \mathrm{msec}$. 
A PSD-93 $\beta$
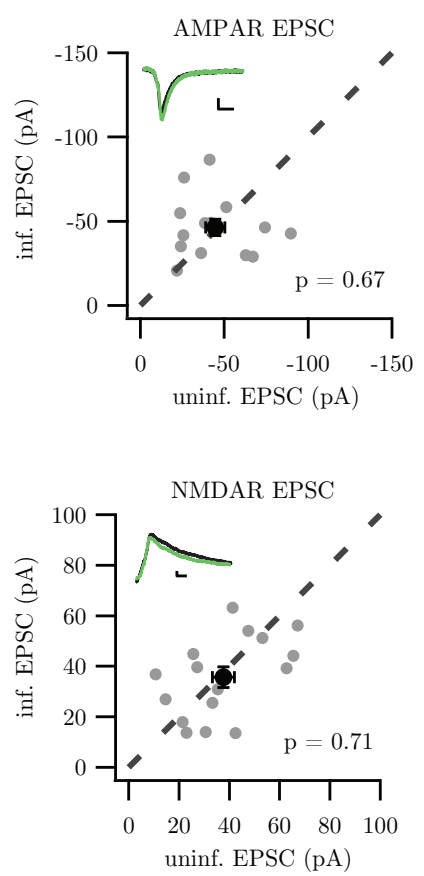

D PSD-93є
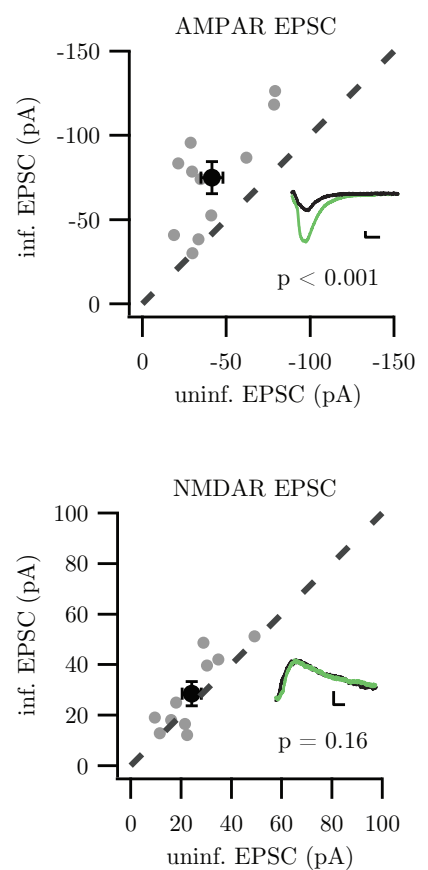

B PSD-93 $\gamma_{1}$

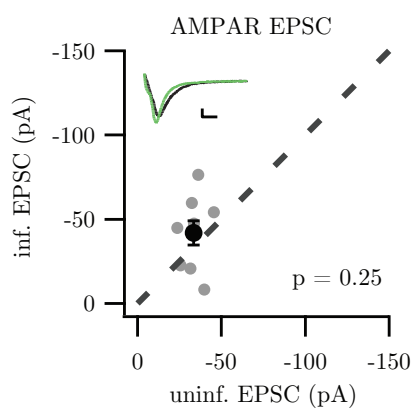

C PSD-93
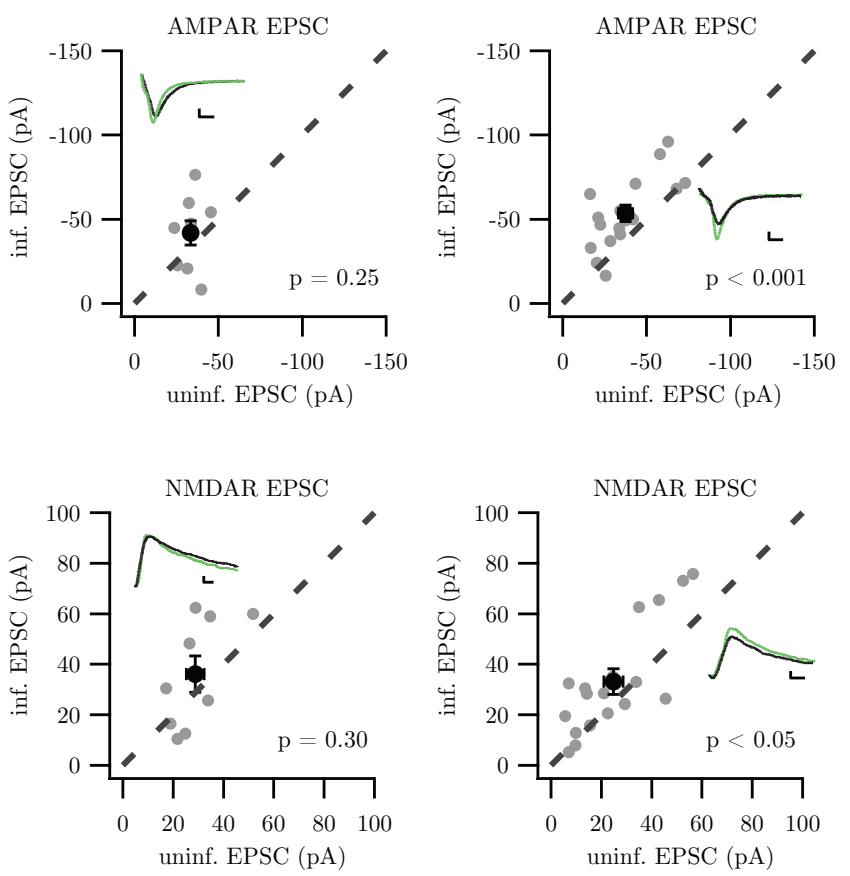

E Summary of overexpression
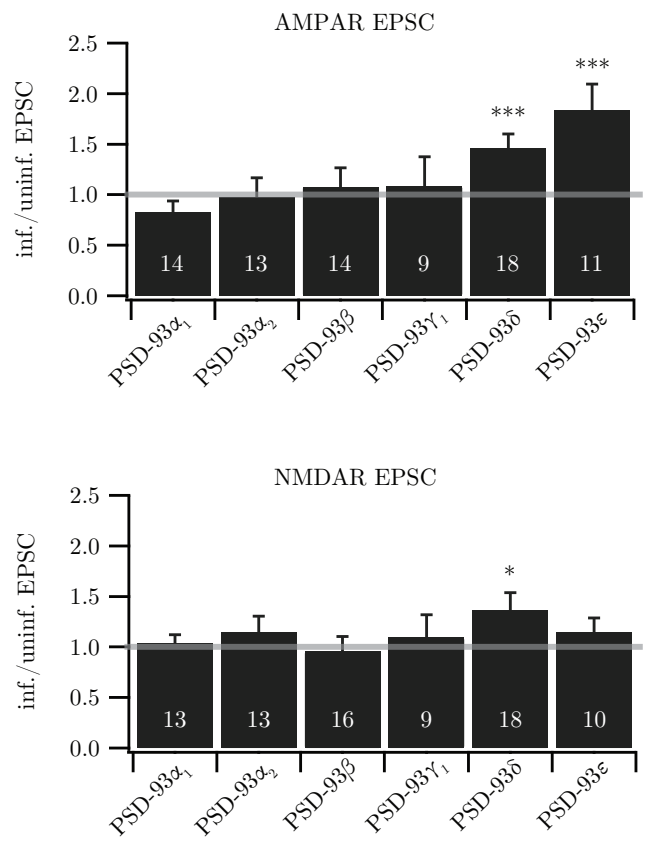


\section{THE ROLE OF PSD-93 IN BASAL SYNAPTIC TRANSMISSION}

AMPAR EPSCs, PSD-93 $\delta$-overexpression also increased the NMDAR-mediated response by $34 \%$ (Figure 4.4C; control: $24.8 \pm 3.8 \mathrm{pA}$, infected: $33.2 \pm 5.1 \mathrm{pA}$ ). Similarly, overexpression of PSD-93 $\epsilon$ led to a significant increase in AMPAR EPSCs (Figure 4.4D; control: $-41.6 \pm 6.5 \mathrm{pA}$, infected: $-75.0 \pm 9.6 \mathrm{pA})$, but no effect on NMDAR EPSCs was observed (control: $24.3 \pm 3.8 \mathrm{pA}$, infected: $28.5 \pm 4.8 \mathrm{pA}$ ). In my hands, similar to PSD93 $\delta$, PSD-95 $\alpha$ overexpression led to an increase in both AMPAR and NMDAR EPSCs (see Figure 4.8A). In fact, it is debated whether PSD-95 manipulations exclusively affect AMPA receptors (Elias et al. 2006; Nakagawa et al. 2004; Schlüter et al. 2006; Schnell et al. 2002), or whether, through transsynaptic crosstalk, release probability is affected that leads to an increase in AMPAR and NMDAR EPSCs (Futai et al. 2007).

Transsynaptic crosstalk could therefore also account for the observed increase in NMDAR EPSCs in case of overexpression of PSD-93 $\delta$. If there is a presynaptic effect, this can be elucidated with paired pulse stimulation. At excitatory synapses in the hippocampus, the second stimulus results in an increased response, a phenomenon that is known as paired pulse facilitation $(\mathrm{PPF})$. PPF is calcium-dependent and has a presynaptic origin, but it is debated whether it results from residual calcium or saturation of calcium buffers (Zucker and Regehr 2002). Changes in PPF are associated with a presynaptic alteration, e.g. a change in transmitter release probability.

Figure 4.5 (facing page): Overexpressing the $\delta$-isoform increases not only AMPAR EPSCs, but also affects a conductance other than NMDARs. Control cell (black), PSD-93 -overexpressing neuron (green). (A) Top: Paired pulse ( $\Delta \mathrm{t}=100 \mathrm{msec}$ ) sample traces for control cell and PSD-93 -expressing neuron. Bottom: The gray traces show the relationship between control cell PPR and infected cell PPR of single experiments, and the black trace the average of the $\mathrm{N}=5$ experiments. (B) Top: I-V relationship of control and PSD-93 -expressing neurons. Mean \pm SEM is plotted; for control, SEM is less than the diameter of the symbol. Bottom: $\mathrm{I}-\mathrm{V}$ curve scaled to the response at $-60 \mathrm{mV}$ shows that there is no difference in rectification between control and PSD-93 $\delta$-expressing neurons. (C) Top: Average traces when cells are voltage-clamped at $+40 \mathrm{mV}$ in the absence $(\mathrm{N}=8)$ or presence $(\mathrm{N}=7)$ of $10 \mu \mathrm{M}$ NBQX. Bottom: The gray traces show the relationship between control and infected cell of single experiments, and the black trace the average for control and infected cell in the absence (left, $\mathrm{N}=18$ ) and presence (right, $\mathrm{N}=7$ ) of $10 \mu \mathrm{M}$ NBQX. (D) Sample traces of control and PSD-93 $\delta$-expressing neurons recorded at $-60 \mathrm{mV}$ and $+40 \mathrm{mV}$ in the presence of $50 \mu \mathrm{M} \mathrm{AP}-\mathrm{V}$, and in the absence (left) or presence (right) of $25 \mu \mathrm{M}$ GYKI53655 (LY303070). n.s. $\mathrm{p} \geq 0.05,{ }^{*} \mathrm{p}<0.05$. Scale bars $10 \mathrm{pA}, 10 \mathrm{msec}$. 
A PPR
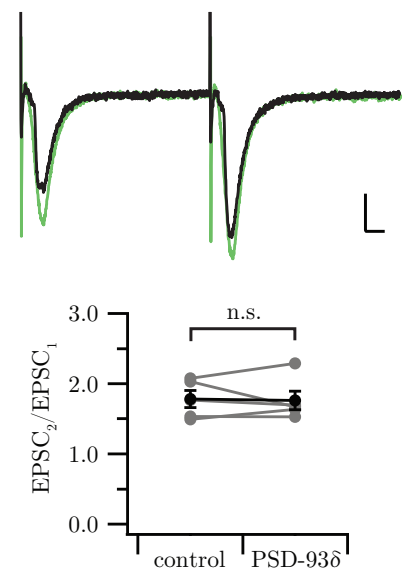

B I-V-plot $(50 \mu \mathrm{M}$ AP-V)
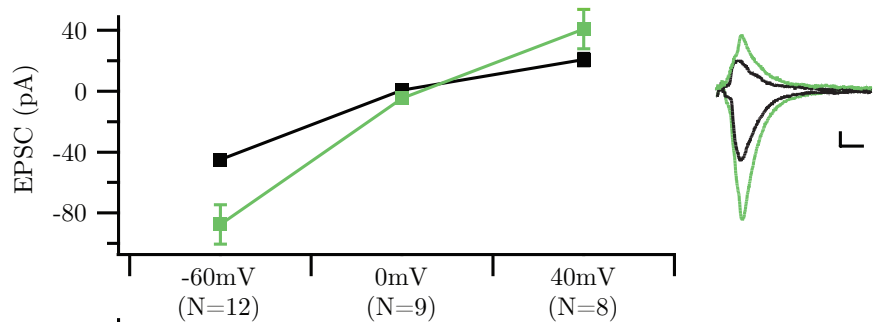

C NBQX

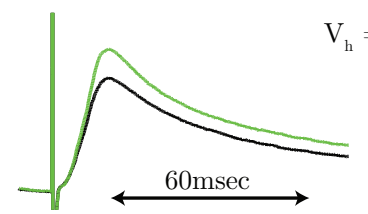

$\mathrm{V}_{\mathrm{h}}=+40 \mathrm{mV}$
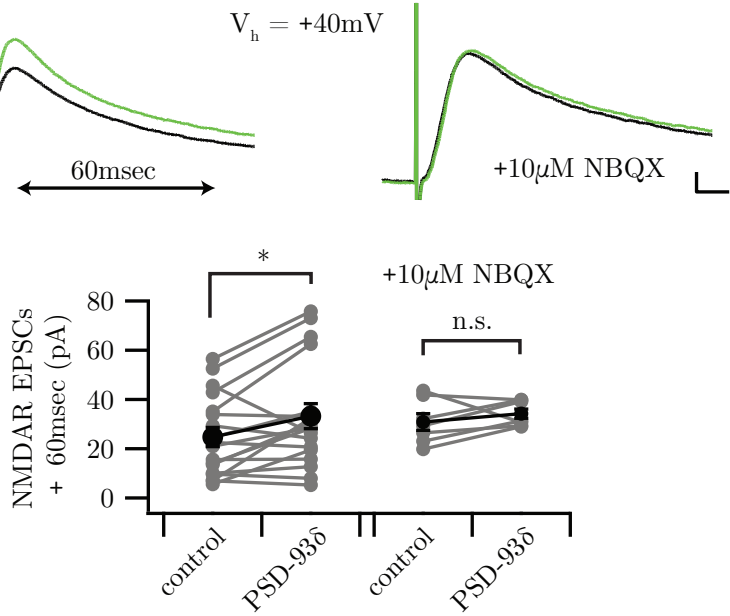

D GYKI

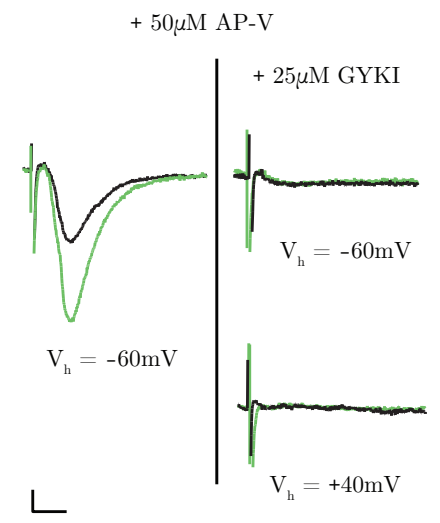




\section{THE ROLE OF PSD-93 IN BASAL SYNAPTIC TRANSMISSION}

Thus, if overexpression of PSD-93 $\delta$ increases the release probability, the magnitude of PPF should be reduced. Hence, the responses of the infected and control cell to two consecutive stimulations (interstimulus interval $\Delta \mathrm{t}=100 \mathrm{msec}$ ) were measured, and the paired pulse ratio ( $\mathrm{PPR}, \mathrm{PPR}=\mathrm{EPSC}_{2} / \mathrm{EPSC}_{1}$ ) was calculated. A sample trace of such a paired pulse experiment is shown in Figure 4.5A, top. In the line plot in Figure 4.5A, the PPR of control versus infected cell of each single experiment is illustrated, together with the mean of $\mathrm{N}=5$ experiments. One can see that there is no difference between the control and the infected cell (PPR control: $1.78 \pm 0.12$, infected: $1.76 \pm 0.13 ; \mathrm{p}=1.0$; $\mathrm{N}=5$ ). Hence, PSD-93 $\delta$ does not seem to affect the presynaptic release probability.

In addition to a presynaptic effect, there are several other possibilities for changes leading to increases in current at $+40 \mathrm{mV}$. AMPARs in the hippocampus typically contain the GluR2 subunit, thereby pass current at positive membrane potentials and are $\mathrm{Ca}^{2+}$-impermeable. If the proportion of GluR2-containing AMPARs was further increased if PSD-93 $\delta$ is overexpressed, more current would pass at positive membrane potentials thus resulting in the increase in current observed at $+40 \mathrm{mV}$. To test whether the degree of rectification is reduced when PSD-93 $\delta$ is overexpressed, the current-voltage relation of the receptor (illustrated by plotting the I-V curve) was analyzed. To this end, recordings were performed in the presence of $50 \mu \mathrm{M}$ AP-V to block NMDA receptors. The evoked AMPAR EPSCs were measured when clamping the cell at $-60 \mathrm{mV}, 0 \mathrm{mV}$, and $+40 \mathrm{mV}$. Still, no change in the rectification was observed (Fig. 4.5B).

As a third possibility, PSD-93 $\delta$ overexpression might indeed lead to an increase in the number of NMDA receptors at the synapse and thus result in the observed increased response. However, when recordings were performed in the presence of $10 \mu \mathrm{M}$ NBQX, an antagonist of both AMPA and kainate receptors, there was no difference between the control and the infected cell (Fig. 4.5C). From this result one can conclude that there is no increase in the synaptic NMDAR number or a change in their conductance of the cells overexpressing PSD-93 $\delta$.

Another scenario is that kainate receptors are recruited into the synapses when overexpressing PSD-93 $\delta$. KARs are activated by glutamate, and the ion channel passes sodium and potassium ions. The conductance of KARs is similar to AMPARs, but kainate receptors inactivate much faster, although in CA1 interneurons slower rise and decay times have been described (Cossart et al. 2002; Frerking and Nicoll 2000). The kainate receptor subunit GluR6 has been shown to be expressed postsynaptically in 
CA1 pyramidal cells (Bureau et al. 1999), and a kainate receptor mediated current can be detected by application of exogenous agonists (Bureau et al. 1999). However, KARs are not activated by stimulation of the Schaffer collateral pathway (Bureau et al. 1999; Castillo et al. 1997). In order to test whether PSD-93 $\delta$ overexpression leads to an incorporation of kainate receptors which can be detected by synaptic stimulation, AMPA and NMDA receptors were pharmacologically blocked with $50 \mu \mathrm{M}$ AP-V and $25 \mu \mathrm{M}$ GYKI53655 (LY303070), a specific AMPAR antagonist. If the increase at $+40 \mathrm{mV}$ is due to the presence of kainate receptors, this effect should still be observed in the presence of the drugs. However, as can be seen in Figure 4.5D, there is no current left when both AMPARs and NMDARs are pharmacologically blocked.

Hence, the nature of the increase in conductance observed at $+40 \mathrm{mV}$ when overexpressing PSD-93 $\delta$ remains unknown.

\subsection{Analyzing the molecular structure of PSD-93}

It was surprising to see that overexpression of the $\alpha$-isoforms of PSD-93 does not increase AMPAR EPSCs as was expected from the overexpression of the $\alpha$-isoforms of PSD-95 and SAP97 (Schlüter et al. 2006). In order to find the reason for this divergence, I undertook a detailed analysis of the N-termini of the $\alpha$-isoforms.

\subsubsection{Exon 3 of PSD-93}

Downstream of exon 3 the isoforms of PSD-93 are identical. PSD-93 $\beta$ differs from all other isoforms in that it does not contain exon 3 (Fig. 3.2 and 3.9), a sequence which is rather conserved among the PSD-MAGUKs. The N-termini of SAP97 and PSD-95 are spliced onto exon 2 and from there on the amino acid sequence is identical for the $\alpha$ - and $\beta$-isoforms of each protein. In order to elucidate whether exon 3 changes the function of the PSD-93 isoforms, mutant constructs of PSD-93 $\alpha_{2}$ and PSD-93 $\beta$ were generated which lacked or contained exon 3. PSD-93 $\alpha_{2}$ without exon 3 did not differ significantly from the control cell as can be seen in Figure 4.6A (AMPAR EPSCs control: $-57.9 \pm 6.4 \mathrm{pA}$, infected: $-69.5 \pm 10.4 \mathrm{pA}$; NMDAR EPSCs control: $38.5 \pm 5.3 \mathrm{pA}$, infected: $29.8 \pm 4.6 \mathrm{pA}$ ). There was no difference in the parameters of basal synaptic transmission between PSD-93 $\alpha_{2}$ with and without exon 3. Likewise, PSD-93 $\beta$ containing exon 3 did not differ from the control cell (AMPAR EPSCs control: $-52.5 \pm 4.8 \mathrm{pA}$, 


\section{THE ROLE OF PSD-93 IN BASAL SYNAPTIC TRANSMISSION}

infected: $-50.8 \pm 5.4 \mathrm{pA}$; NMDAR EPSCs control: $31.5 \pm 3.1 \mathrm{pA}$, infected: $24.2 \pm 4.5 \mathrm{pA}$ ), and behaved exactly as overexpressing the original PSD-93 $\beta$ construct lacking exon 3 (Fig. 4.6B). Thus, exon 3 does not seem to participate in the unexpected behavior of the $\alpha$-isoforms of PSD-93.

A PSD- $93 \alpha_{2}$ w/o exon 3
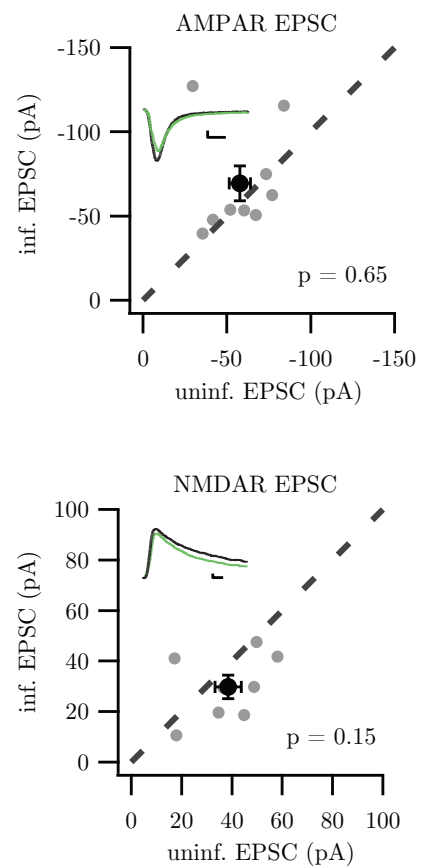

B PSD-93 $\beta \mathrm{w} /$ exon 3
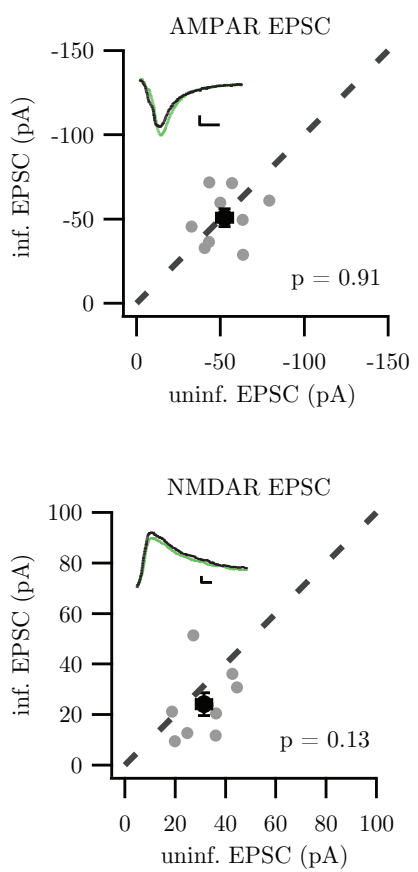

Figure 4.6: Exon 3 does not immediately affect basal synaptic transmission. (A) Scatter plot of amplitudes of cells expressing PSD-93 $\alpha_{2}$ lacking exon 3 and neighboring control neurons (top: AMPAR EPSCs, $\mathrm{N}=9$; bottom: NMDAR EPSCs, N=8). Sample traces of infected (green) and control cell (black). (B) Scatter plot of amplitudes of cells expressing PSD-93 $\beta$ containing exon 3 and neighboring control neurons (top: AMPAR EPSCs, N=9; bottom: NMDAR EPSCs, N=9). Sample traces of infected (green) and control cell (black). Scale bars 10pA, 10msec.

\subsubsection{The $\alpha$-N-terminus of PSD-93}

Since exon 3 could be ruled out, I asked whether there is something intrinsic in the first two N-terminal exons of PSD-93 $\alpha_{1}$ or $\alpha_{2}$ that prevents it from behaving like a classical $\alpha$-isoform. Although the $\alpha$-N-termini of the PSD-MAGUKs all contain a cysteine doublet that is palmitoylated, there is still considerable difference both in exon 1 and 
in exon 2 downstream of the KYRY consensus (Fig. 3.9). Two possibilities exist why PSD-93 $\alpha_{1}$ and $\alpha_{2}$ do not enhance AMPAR EPSCs: First, exon 1, although appearing like a bona fide $\alpha$-exon, is not functional. Second, PSD-93 contains an inhibitory domain that overwrites the AMPAR-enhancing action of exon 1. It is unlikely that such an inhibitory domain is present in the C-terminus of PSD-93, since PSD-93 $\delta$ and PSD-93 $\epsilon$ lead to an increase in AMPAR EPSCs.

In order to distinguish between these two possibilities, I generated chimeric constructs of PSD-95 $\alpha$ and PSD-93 $\alpha_{2}$. In one set of constructs, the full N-terminus from the start codon to PDZ1 was exchanged between PSD-95 $\alpha$ and PSD-93 $\alpha_{2}$. These constructs have been termed $95 \mathrm{~Np} 93$ and $93 \mathrm{~Np} 95$, respectively. In a second set of constructs, only exon 1 was exchanged. These are the first twenty amino acids in case of PSD-93 $\alpha_{2}$, and the first nine amino acids in the case of PSD-95 $\alpha$. Hence, these constructs have been termed $93_{1-20} \mathrm{p} 95$ and $95_{1-9} \mathrm{p} 93$. A schematic can be found in Figure 4.7D and 4.8D.

I find that exon 1 of PSD-93 $\alpha_{2}$ in principle behaves like a classical $\alpha$-isoform and is as functional as exon 1 of PSD-95. The construct 931-20p95 increases AMPAR EPSCs more than 2-fold when compared to the neighboring control cell (Fig. 4.7C; control: $-44.0 \pm 4.9 \mathrm{pA}$, infected: $-96.3 \pm 19.4 \mathrm{pA})$. In contrast, overexpressing a construct in which the full N-terminus of PSD-95 upstream of PDZ1 is exchanged with that of PSD-93 $\alpha_{2}$ (93Np95), AMPAR EPSCs are not different from the control cell (Fig. 4.7B; control: $-65.9 \pm 3.6 \mathrm{pA}$, infected: $-66.6 \pm 8.1 \mathrm{pA})$. In both cases, NMDAR-mediated EPSCs are not affected by the manipulation $\left(93_{1-20} \mathrm{p} 95\right.$ control: $32.2 \pm 3.9 \mathrm{pA}$, infected: $37.6 \pm 4.5 \mathrm{pA}$; 93Np95 control: $31.1 \pm 2.7 \mathrm{pA}$, infected: $33.2 \pm 4.3 \mathrm{pA})$. The NMDAR responses do not differ across constructs (Kruskal-Wallis test, $\mathrm{p}=0.44$ ). These data are summarized in the bar graph in Figure 4.7E. Exon 1 of PSD-93 $\alpha_{2}$ is functional and can in principle increase AMPAR EPSCs by 2-fold, similar to PSD-95 $\alpha$ (Fig. 4.8A). Yet, since 93Np95 does not increase AMPAR EPSCs, there is a domain in the sequence from exon 2 to PDZ1 of PSD-93 which inhibits the $\alpha$-like behavior of exon 1.

Similarly, only the full N-terminus of PSD-95 upstream of PDZ1 and not just exon 1 can convert PSD-93 into a classical $\alpha$-isoform. As shown previously, PSD-95 overexpression results in a 2-fold increase in AMPAR EPSCs as can be seen in Figure 4.8A (control: $-43.5 \pm 3.5 \mathrm{pA}$, infected: $-96.6 \pm 13.8 \mathrm{pA}$ ). Along the lines with the results shown 
A PSD-93 $\alpha_{2}$
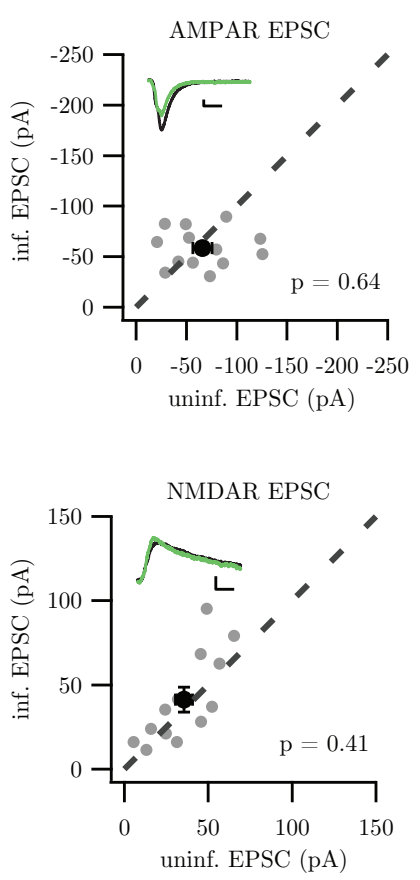

D Schematic of constructs

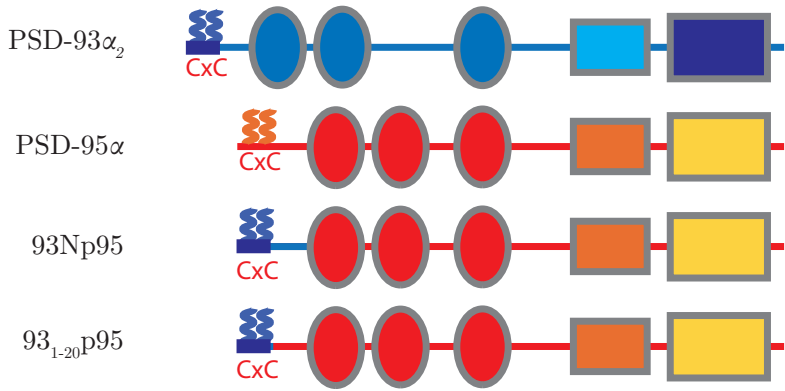

C 931-20p95
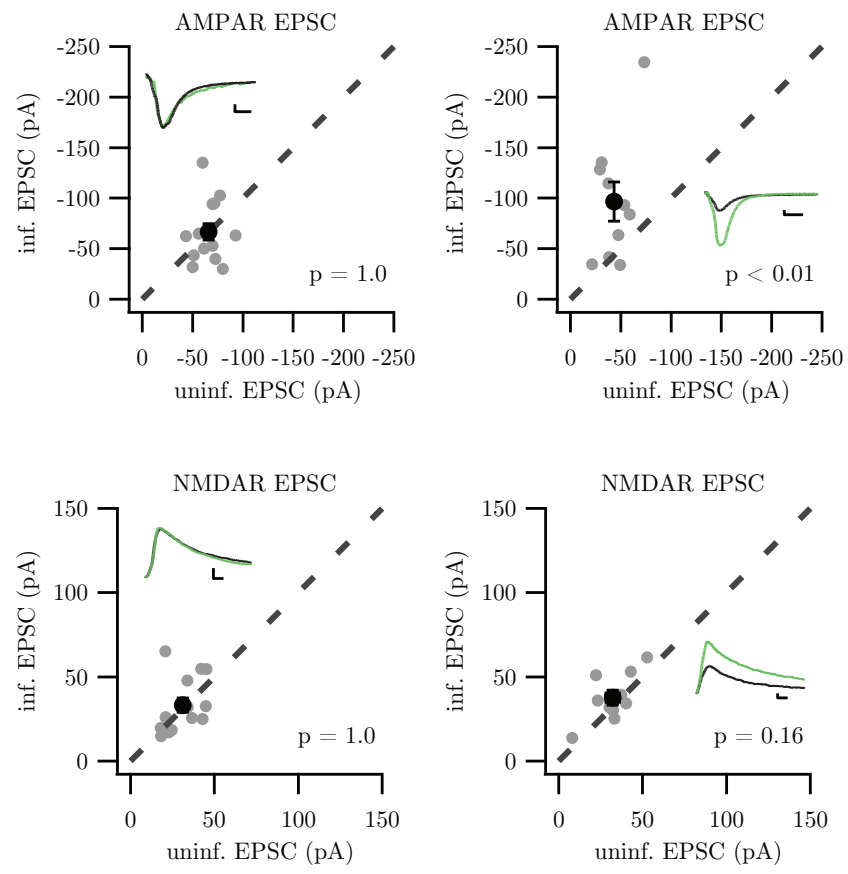

$\mathrm{E}$

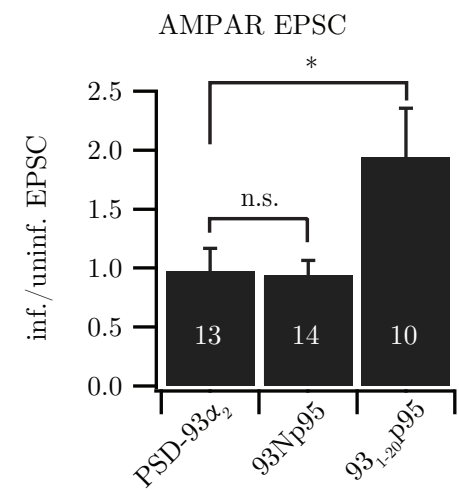

Figure 4.7: Exon 1 of PSD-93 $\alpha_{2}$ is functional and increases AMPAR EPSCs. (A) Amplitudes of cells expressing PSD-93 $\alpha_{2}$ versus neighboring control neurons (top: AMPAR EPSCs, N=13; bottom: NMDAR EPSCs, N=13). Sample traces of infected (green) and control cell (black). (B) Amplitudes of cells expressing 93Np95 versus neighboring control neurons (top: AMPAR EPSCs, N=14; bottom: NMDAR EPSCs, N=14). Sample traces of infected (green) and control cell (black). (C) Scatter plot of amplitudes of cells expressing 931-20p95 versus control neurons (top: AMPAR EPSCs, N=10; bottom: NMDAR EPSCs, $\mathrm{N}=10$ ). Sample traces of infected (green) and control cell (black). (D) Schematic of the constructs used. (E) Bar graph comparing the different constructs. PSD-93 $\alpha_{2}$ and $93 \mathrm{~Np} 95$ do not differ significantly $(\mathrm{p}=0.91)$, but PSD-93 $\alpha_{2}$ and $93_{1-20} \mathrm{p} 95$ do $(\mathrm{p}=0.01)$. n.s. $\mathrm{p} \geq 0.05$, ${ }^{*} \mathrm{p}<0.05$. Scale bars $10 \mathrm{pA}, 10 \mathrm{msec}$. 
A PSD-95 $\alpha$
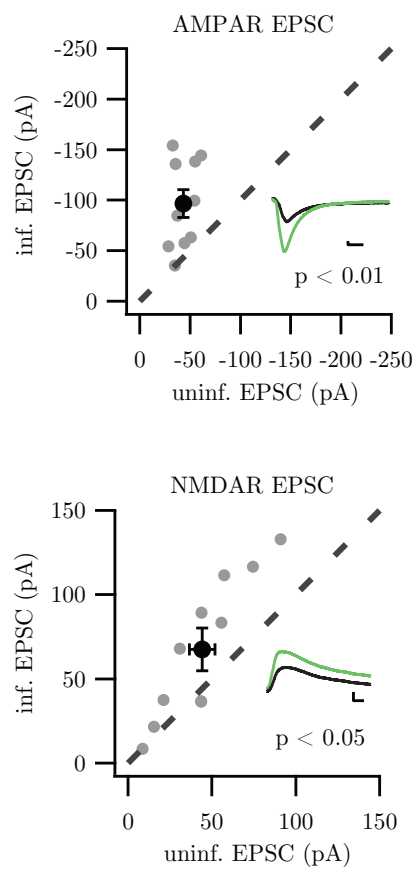

D Schematic of constructs

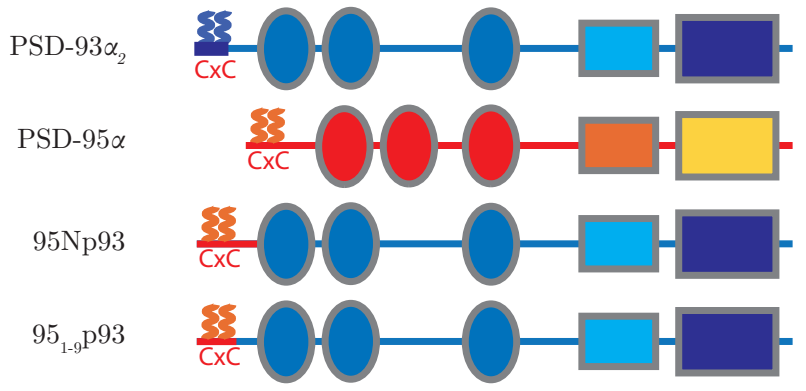

B $95 \mathrm{~Np} 93$
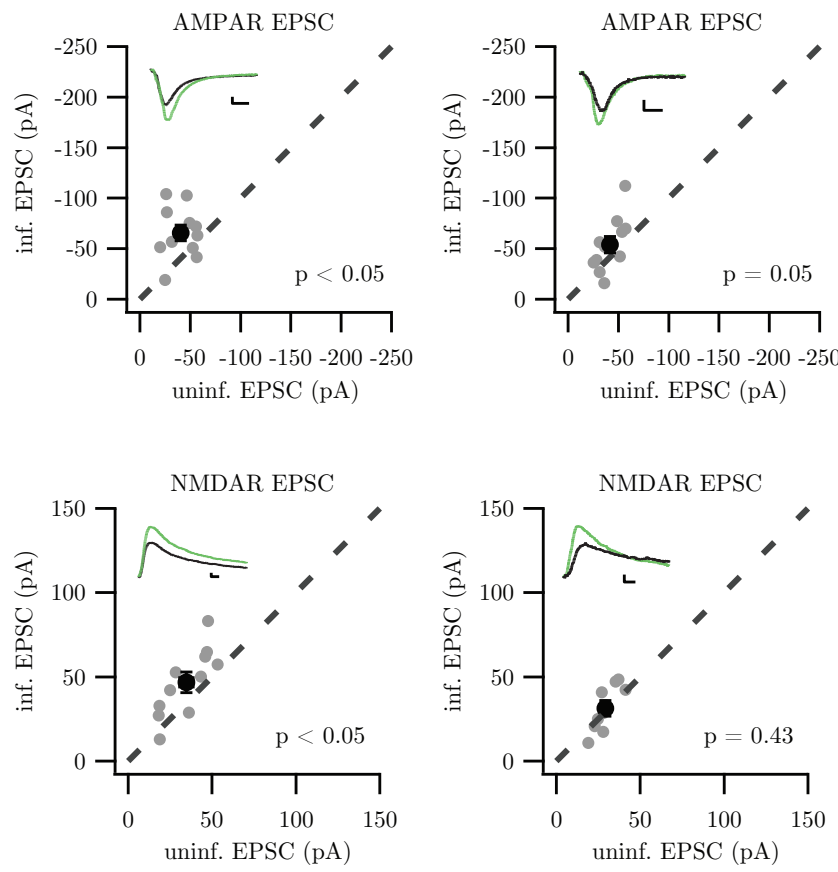

$\mathrm{E}$

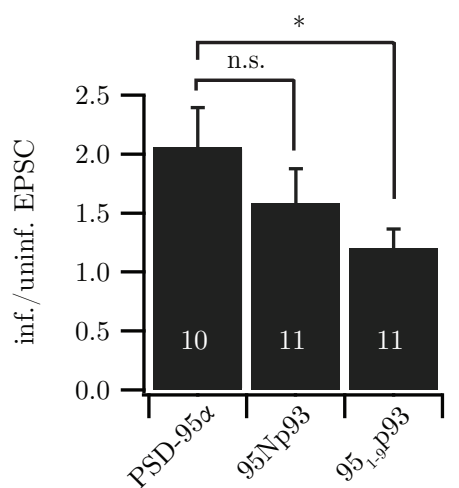

Figure 4.8: The full N-terminus of PSD-95 $\alpha$, but not exon 1, can convert PSD-93 into a classical $\alpha$-isoform. (A) Amplitudes of cells expressing PSD-95 $\alpha$ versus neighboring control neurons (top: AMPAR EPSCs, $\mathrm{N}=10$; bottom: NMDAR EPSCs, $\mathrm{N}=11$ ). Sample traces of infected (green) and control cell (black). (B) Amplitudes of cells expressing 95Np93 versus neighboring control neurons (top: AMPAR EPSCs, N=11; bottom: NMDAR EPSCs, N=11). Sample traces of infected (green) and control cell (black). (C) Scatter plot of amplitudes of cells expressing 951-9p93 versus control neurons (top: AMPAR EPSCs, N=11; bottom: NMDAR EPSCs, N=9). Sample traces of infected (green) and control cell (black). (D) Schematic of the constructs used. (E) Bar graph comparing the different constructs. PSD-95 and 95Np93 do not differ significantly ( $\mathrm{p}=0.28)$, but PSD-95 and $95_{1-9} \mathrm{p} 93$ do $(\mathrm{p}<0.05)$. n.s. $\mathrm{p} \geq 0.05,{ }^{*} \mathrm{p}<0.05$. Scale bars $10 \mathrm{pA}, 10 \mathrm{msec}$. 


\section{THE ROLE OF PSD-93 IN BASAL SYNAPTIC TRANSMISSION}

in Figure 4.7, AMPAR EPSCs are still significantly increased by $58 \%$ if the full Nterminus of PSD-95 $\alpha$ is placed on the PSD-93 backbone, although the effect is not as strong as with full-length PSD-95 $\alpha$ (Fig. 4.8B; control: $-40.7 \pm 4.4 \mathrm{pA}$, infected: $65.7 \pm 7.8 \mathrm{pA}$ ). If only exon 1 of PSD-95 $\alpha$ and PSD-93 $\alpha_{2}$ are exchanged ( $\alpha$-exon of PSD-95 on PSD-93 backbone, 951-9p93), AMPAR EPSCs are not significantly different in the infected compared to the control cell $(\mathrm{p}=0.05$; control: $-41.5 \pm 3.6 \mathrm{pA}$, infected: $-54.0 \pm 8.1 \mathrm{pA}$ ). These data are summarized in the bar graph in Figure 4.8E. Together with the data in Figure 4.7, these results demonstrate that an N-terminal fragment of PSD-93 downstream of exon 1 acts inhibitory, independent of whether it is paired with exon 1 of PSD-93 $\alpha_{2}$ or PSD-95 $\alpha$.

NMDAR EPSCs are significantly increased when the full N-terminus of PSD-95 is overexpressed, independent whether the full N-terminus is placed on the PSD-95 backbone (Fig. 4.8A; control: $44.2 \pm 7.5 \mathrm{pA}$, infected: $67.5 \pm 12.7 \mathrm{pA}$ ) or the PSD-93 backbone (Fig. 4.8B; control: $34.8 \pm 4.0 \mathrm{pA}$, infected: $46.7 \pm 6.1 \mathrm{pA}$ ). When exon 1 of PSD-95 $\alpha$ pairs with PSD-93 $\alpha_{2}$, NMDAR EPSCs do not differ between the control and the infected cell (control: $29.3 \pm 2.6 \mathrm{pA}$, infected: $31.3 \pm 4.6 \mathrm{pA}$ ). Due to the variability in the data, there is no significant difference in NMDAR EPSCs between the three constructs when assessed with the Kruskal-Wallis test statistic $(\mathrm{p}=0.06)$.

\subsection{All isoforms of PSD-93 can compensate for the acute loss of PSD-95}

Both, knocking down PSD-93 with RNA interference and the genetic knockout of the protein did not impair AMPAR function and therefore did not generate a loss-offunction phenotype. The genetic knockout of PSD-95, on the other hand, has previously shown variable consequences ranging from no effect on parameters of basal synaptic transmission (Migaud et al. 1998), over mild effects (Carlisle et al. 2008), to a strong impairment in a subset of synapses (Béique et al. 2006). In contrast, the acute loss of the protein due to RNA interference consistently reduces AMPAR EPSCs by 50\% (Elias et al. 2006; Nakagawa et al. 2004; Schlüter et al. 2006). Although it is not clear why the results in the KOs are inconsistent, the differences in the results between acute and long-term deletion of PSD-95 led to the speculation that genetic loss of PSD-95 can be compensated for by the other members of the PSD-MAGUK 
family (Elias et al. 2006; Schlüter et al. 2006). In order to test whether all isoforms of PSD-93 or only a subset can balance out the loss of PSD-95, I knocked down PSD95 with an shRNA and simultaneously overexpressed individual isoforms of PSD-93. The knockdown construct for PSD-95, sh95p, has been used successfully in a previous study, and helped to unravel the activity-dependent function of PSD-95 $\beta$ and SAP97 $\beta$ (Schlüter et al. 2006). In my hands, knockdown of PSD-95 led to the previously described 50\% reduction in AMPAR EPSC amplitude without affecting NMDAR EPSCs (Fig. 4.9A; AMPAR EPSCs control: $-46.7 \pm 6.2 \mathrm{pA}$, infected: $-23.8 \pm 2.7 \mathrm{pA}$; NMDAR EPSCs control: $26.2 \pm 4.4 \mathrm{pA}$, infected: $24.0 \pm 2.9 \mathrm{pA}$ ).

Although the $\alpha$-isoforms of PSD-93 do not behave like bona fide $\alpha$-isoforms, they can rescue the reduction in AMPAR EPSCs caused by the knockdown of PSD-95. There is no difference between the control cell and the infected neuron when PSD-95 is replaced by PSD-93 $\alpha_{1}$ (Fig. 4.9B; AMPAR EPSCs control: $-61.7 \pm 8.3 p A$, infected: $-50.0 \pm 7.4 p A$; NMDAR EPSCs control: 34.8 $\pm 5.5 \mathrm{pA}$, infected: $38.6 \pm 6.8 \mathrm{pA}$ ) or PSD-93 $\alpha_{2}$ (Fig. 4.9C; AMPAR EPSCs control: $-51.1 \pm 7.6 \mathrm{pA}$, infected: $-60.1 \pm 6.8 \mathrm{pA}$; NMDAR EPSCs control: $30.9 \pm 6.5 \mathrm{pA}$, infected: $30.1 \pm 5.9 \mathrm{pA})$.

Similar to PSD-95 $\beta$ and SAP97 $\beta$ (Schlüter et al. 2006), PSD-93 $\beta$ also restores AMPAR EPSC amplitudes to wildtype levels (Fig. 4.9D; AMPAR EPSCs control: $-46.7 \pm 5.4 \mathrm{pA}$, infected: $-61.8 \pm 14.4 \mathrm{pA}$; NMDAR EPSCs control: $29.3 \pm 4.3 \mathrm{pA}$, infected: $26.9 \pm 3.9 \mathrm{pA}$ ).

Likewise, PSD-93 $\gamma_{1}$ rescues the knockdown of PSD-95 such that AMPAR EPSCs do not differ between the control and the infected neuron (Fig. 4.9E; AMPAR EPSCs control: $-39.8 \pm 3.4 \mathrm{pA}$, infected: $-39.3 \pm 6.4 \mathrm{pA}$; NMDAR EPSCs control: $29.7 \pm 3.4 \mathrm{pA}$, infected: $26.9 \pm 1.6 \mathrm{pA})$.

PSD-938, on the other hand, not only rescues the reduction of AMPAR EPSC amplitude to control levels (control: $-50.5 \pm 3.9 \mathrm{pA}$, infected: $-60.1 \pm 6.6 \mathrm{pA} ; \mathrm{p}=0.15$ ), but at the same time leads to a significant 1.52-fold increase in NMDAR EPSCs (control: 29.18 $\pm 3.84 \mathrm{pA}$, infected: 44.49 $\pm 7.12 \mathrm{pA} ; \mathrm{p}<0.005)$ as shown in Figure 4.10A. Although the effect on AMPAR EPSCs of overexpression of PSD-93 $\delta$ and replacement of PSD95 with PSD-93 is not statistically different (Fig. 4.10C; $\mathrm{p}=0.22$ ), there is a strong indication that the two conditions actually differ. When compared to the neighboring control cell, PSD-93 $\delta$ overexpression leads to a significant increase in AMPAR EPSCs 

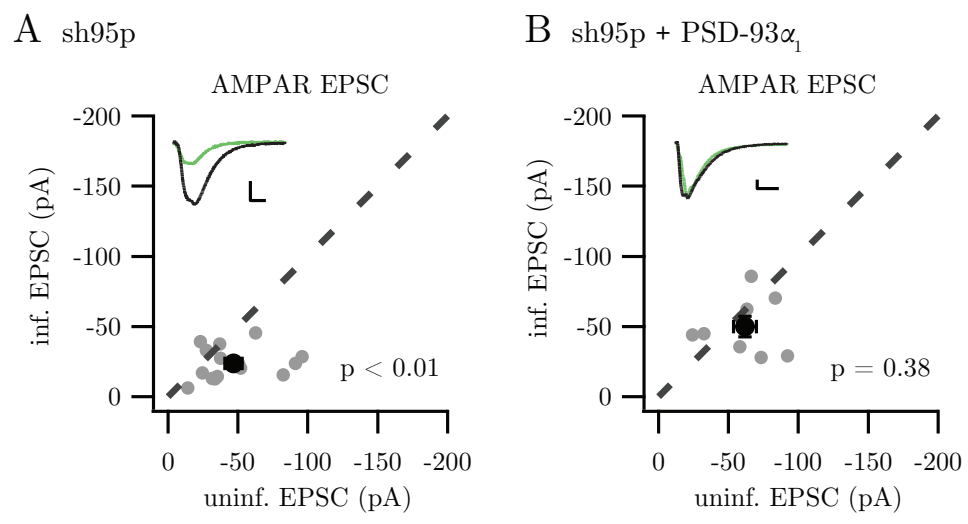

C $\operatorname{sh} 95 p+$ PSD-93 $\alpha_{2}$
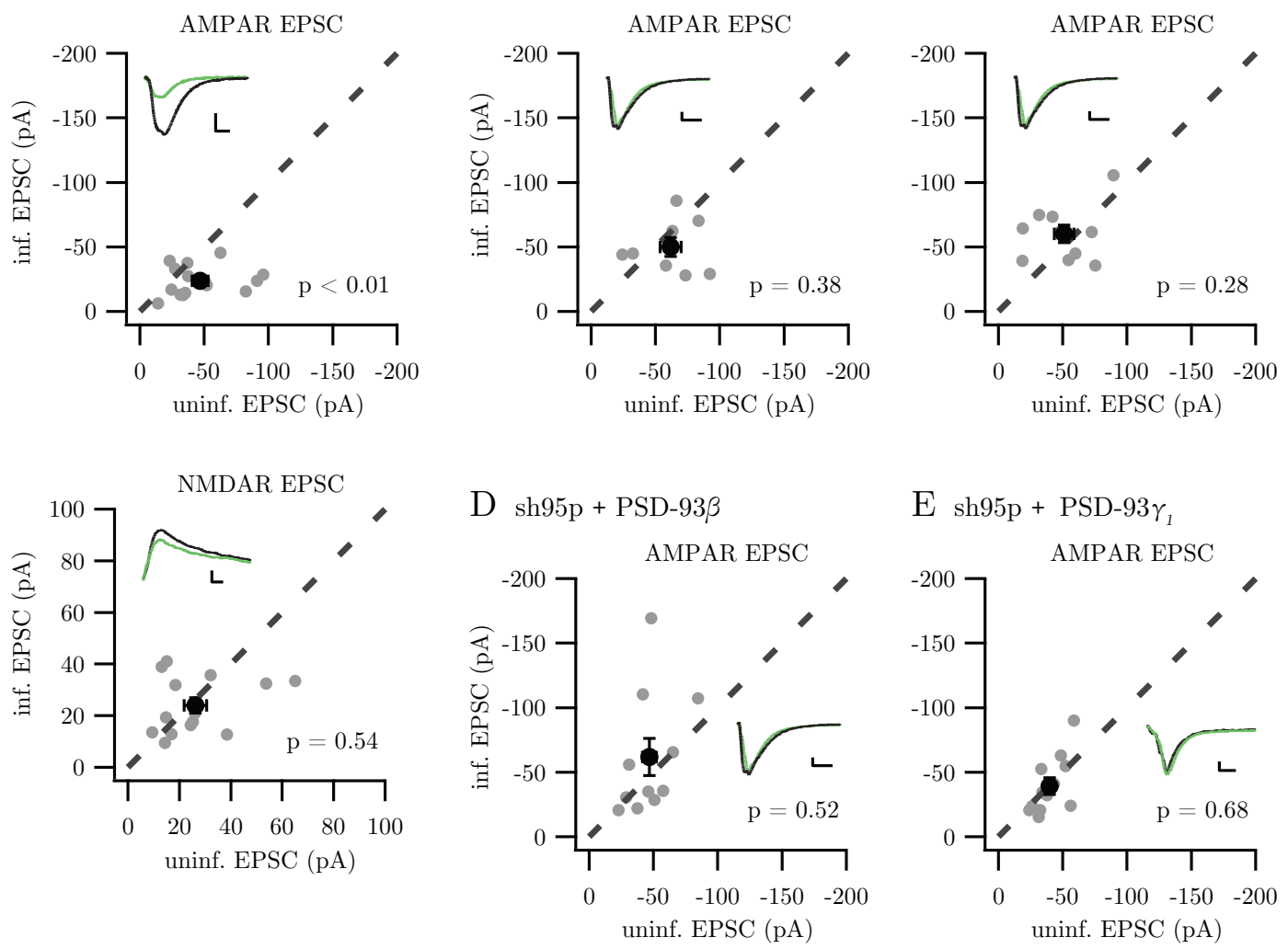

Figure 4.9: PSD-93 can rescue the acute loss of PSD-95. Endogenous PSD-95 was knocked down via RNA interference with the construct sh95p. (A) Amplitudes of neurons expressing the knockdown construct sh95p plotted against neighboring control cells. Top: AMPAR EPSCs $(\mathrm{N}=16)$, bottom: NMDAR EPSCs $(\mathrm{N}=14)$. (B) Scatter plot of AMPAR EPSCs in cells expressing sh95p and overexpressing PSD-93 $\alpha_{1}$ versus control cells $(\mathrm{N}=8)$. (C) Scatter plot of AMPAR EPSCs in cells expressing sh95p and overexpressing PSD-93 $\alpha_{2}$ versus control cells $(\mathrm{N}=10)$. (D) Scatter plot of AMPAR EPSCs in cells expressing sh95p and overexpressing PSD-93 $\beta$ versus control cells $(\mathrm{N}=11)$. (E) Scatter plot of AMPAR EPSCs in cells expressing sh95p and overexpressing PSD-93 $\gamma_{2}$ versus control cells $(\mathrm{N}=12)$. Sample traces of infected (green) and control cell (black) are shown in all subfigures. Scale bars 10pA, 10msec.

(see Fig. 4.4C, $\mathrm{p}<0.001$ ), whereas replacement of PSD-95 with PSD-93 $\delta$ results only in the rescue of AMPAR EPSCs to control levels (Fig. 4.10A, p=0.15).

In contrast, the $\epsilon$-isoform increases AMPAR EPSCs even in the absence of PSD-95, while NMDAR EPSCs do not change in a statistically significant manner (Fig. 4.10B). 
A sh95p + PSD-93
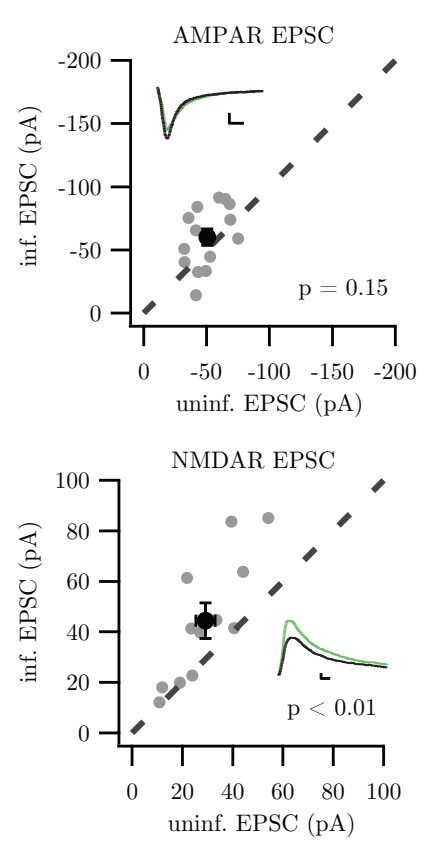

B sh95p + PSD-93€
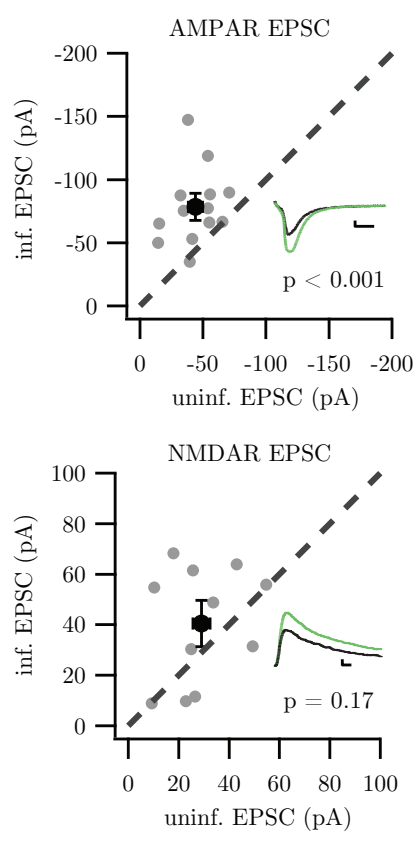

C
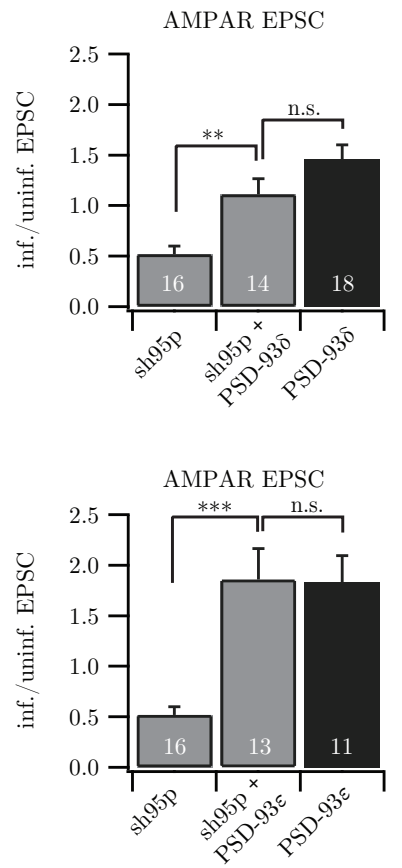

Figure 4.10: Replacement of PSD-95 with PSD-93 $\delta$ and PSD-93 $\epsilon$. (A) Effect of knockdown of PSD-95 and overexpression of PSD-93 $\delta$ versus control shown in a scatter plot of amplitudes of AMPAR EPSCs (top, N=14) and NMDAR EPSCs (bottom, N=12). Sample traces of infected (green) and control cell (black). (B) Effect of knockdown of PSD95 and overexpression of PSD-93 $\epsilon$ versus control shown in a scatter plot of amplitudes of AMPAR EPSCs (top, N=13) and NMDAR EPSCs (bottom, N=11). Sample traces of infected (green) and control cell (black). (C) Summary of effects on AMPAR EPSCs for PSD-93 (top) and PSD-93 $\epsilon$ (bottom) overexpression or replacement of PSD-95. n.s. $\mathrm{p} \geq 0.05,{ }^{* *} \mathrm{p}<0.01,{ }^{* * *} \mathrm{p}<0.001$. Scale bars $10 \mathrm{pA}, 10 \mathrm{msec}$. 


\section{THE ROLE OF PSD-93 IN BASAL SYNAPTIC TRANSMISSION}

Compared to the control cell, AMPAR EPSCs are almost 2-fold higher (AMPAR EPSCs control: $-43.9 \pm 4.8 \mathrm{pA}$, infected: $-78.4 \pm 8.2 \mathrm{pA}$; NMDAR EPSCs control: $29.0 \pm 4.5$, infected: $40.5 \pm 6.9 \mathrm{pA})$. The increase in AMPAR EPSCs seems to be independent of the presence or absence of PSD-95, as there is no statistical difference between the

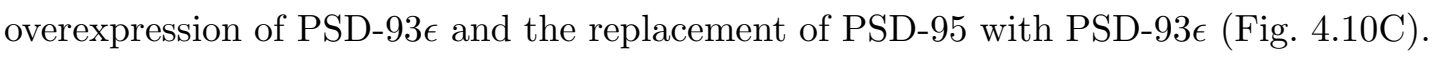

\subsection{Activity manipulations show distinct properties of the PSD-93 isoforms}

Previous work has shown that both the magnitude of the reduction of AMPAR EPSCs due to the knockdown of PSD-95, as well as the increase of AMPAR EPSCs when replacing it with PSD-95 $\beta$ or SAP97 $\beta$ depends of the activity of the culture and requires NMDAR activation (Schlüter et al. 2006). Bicuculline (BIC), a GABA A $_{\text {chnel }}$ inhibitor, can be used to increase the spontaneous excitatory synaptic activity in the slice cultures. I aimed to test whether the PSD-93 isoforms show activity dependent behavior similar to the $\beta$-isoforms of PSD-95 and SAP97, or whether they are not affected by activity, as are the $\alpha$-isoforms of PSD-95 and SAP97 (Schlüter et al. 2006).

From the day of injection on, the slice culture medium was supplemented with $20 \mu \mathrm{M}$ BIC. Overexpressing PSD-93 $\alpha_{1}$, PSD-93 $\alpha_{2}$, or PSD-93 $\gamma_{1}$ in the absence of PSD95 while increasing excitatory activity in the culture does not affect AMPAR EPSCs or NMDAR EPSCs (Fig. 4.11). There is no difference between the EPSC amplitudes in the control cell compared to the infected neighbor for PSD-93 $\alpha_{1}$ (AMPAR EPSCs control: $-52.3 \pm 7.6 \mathrm{pA}$, infected: $-52.6 \pm 9.9 \mathrm{pA}$; NMDAR EPSCs control: $35.9 \pm 4.3$, infected: $45.2 \pm 4.0 \mathrm{pA}$ ), PSD-93 $\alpha_{2}$ (AMPAR EPSCs control: $-65.9 \pm 13.8 \mathrm{pA}$, infected: $-58.9 \pm 7.6 \mathrm{pA}$; NMDAR EPSCs control: $30.4 \pm 4.5$, infected: $31.1 \pm 5.7 \mathrm{pA}$ ), or PSD$93 \gamma_{1}$ (AMPAR EPSCs control: $-28.7 \pm 3.6 \mathrm{pA}$, infected: $-43.3 \pm 6.1 \mathrm{pA}$; NMDAR EPSCs control: $23.3 \pm 5.2$, infected: $31.9 \pm 4.6 \mathrm{pA}$ ).

In contrast, as shown in Figure 4.12A and D, there is a strong 4-fold increase of AMPAR EPSCs when PSD-93 $\beta$ is overexpressed and the excitatory activity in the culture dish is increased by blocking inhibitory transmission (AMPAR EPSCs control: $-27.1 \pm 2.5 \mathrm{pA}$, infected: $-113.9 \pm 16.2 \mathrm{pA}$; NMDAR EPSCs control: $16.7 \pm 3.5$, infected: $24.2 \pm 2.2 \mathrm{pA}, \mathrm{p}=0.11$ ). Surprisingly, this effect is not as strong when the activity manipulation in the culture dish is not chronic. Blocking inhibitory transmis- 
A $\operatorname{sh} 95 p+$ PSD- $93 \alpha_{1}$

$+\mathrm{BIC}$
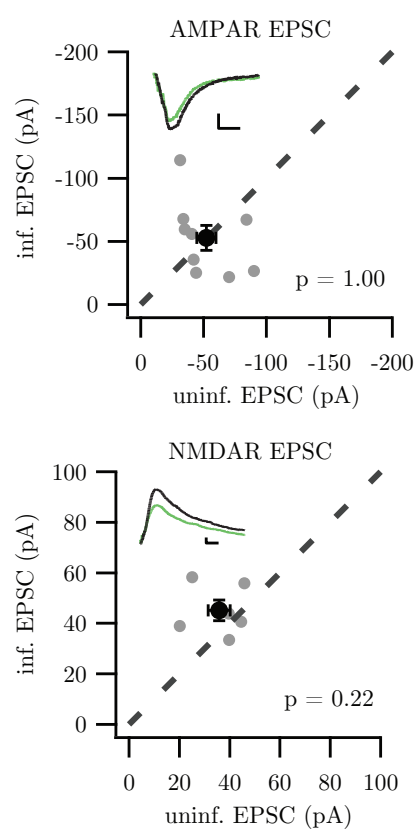

B sh95p + PSD-93 $\alpha_{2}$

$+\mathrm{BIC}$
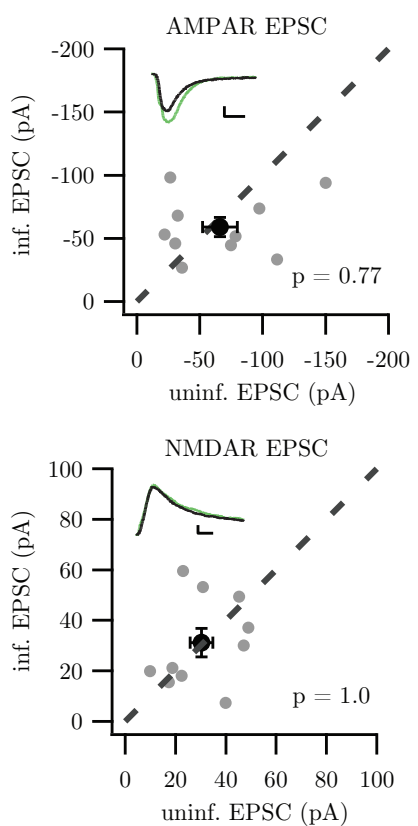

C sh95p + PSD-93 $\gamma_{1}$

$+\mathrm{BIC}$
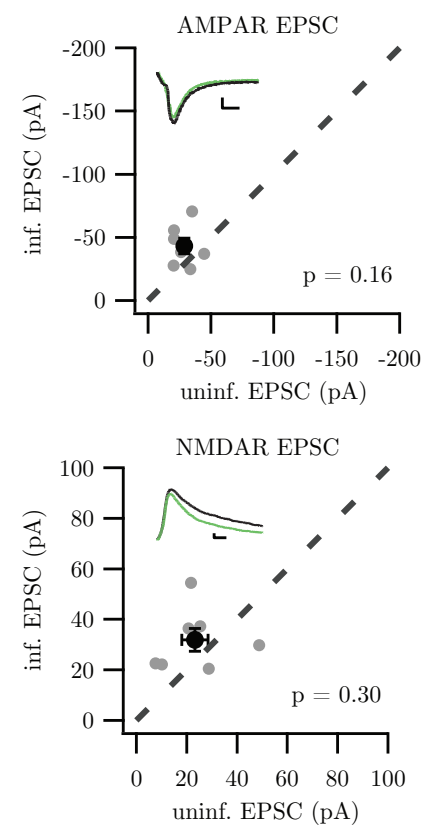

Figure 4.11: PSD-93 $\alpha_{1}$, PSD-93 $\alpha_{2}$, and PSD-93 $\gamma_{1}$ do not show activitydependent behavior. Scatter plot of amplitudes of infected cells versus control neurons for AMPAR EPSCs (top) and NMDAR EPSCs (bottom). Excitatory activity in the culture was increased by supplementing the medium with $20 \mu \mathrm{M}$ BIC. In the infected cells, endogenous PSD-95 was knocked down with sh95p. Simultaneously, the following isoforms of PSD-93 were overexpressed: (A) PSD-93 $\alpha_{1}$ (N=9/6, AMPAR/NMDAR), (B) PSD-93 $\alpha_{2}$ $(\mathrm{N}=10 / 10)$, (C) PSD-93 $\gamma_{1}(\mathrm{~N}=9 / 7)$. Sample traces of infected (green) and control cell (black). Scale bars 10pA, 10msec. 


\section{THE ROLE OF PSD-93 IN BASAL SYNAPTIC TRANSMISSION}

sion for two days only leads to a 1.7-fold, but still significant increase in AMPAR EPSCs compared to control neurons in the same slice (Fig. 4.12B; AMPAR EPSCs control: $-42.0 \pm 5.1 \mathrm{pA}$, infected: $-69.9 \pm 8.7 \mathrm{pA}$; NMDAR EPSCs control: $12.8 \pm 2.3$, infected: $14.6 \pm 1.3 \mathrm{pA}, \mathrm{p}=0.58)$. However, this two-day manipulation is significantly different from the long-term manipulation (Fig. 4.12D). The increase in AMPAR EPSCs by overexpressing PSD-93 $\beta$ and long-term blockage of inhibitory transmission is dependent on the activity of NMDA receptors. When blocking NMDA receptors with the NMDAR antagonist AP-V $(25 \mu \mathrm{M})$ for two days before recording, no difference between control and infected neurons is observed (Fig. 4.12C, D; AMPAR EPSCs control: $-57.8 \pm 6.8 \mathrm{pA}$, infected: $-75.2 \pm 12.2 \mathrm{pA}$; NMDAR EPSCs control: 27.8 \pm 9.8 , infected: $28.5 \pm 8.1 \mathrm{pA}, \mathrm{p}=0.81)$. NMDAR EPSCs do not differ between the tested conditions (Fig. 4.12D). The behavior of PSD-93 $\beta$ thus resembles that of PSD-95 $\beta$ and SAP97 $\beta$ which have been shown to be modulated by activity in an NMDAR-dependent manner (Schlüter et al. 2006).

In addition, PSD-93 $\delta$ also appears to be dependent on the excitatory activity in the slice culture. Acute knockdown of PSD-95 could be rescued to control levels when PSD-93 $\delta$ was overexpressed (see Fig. 4.10A and Fig. 4.13C). However, when inhibitory synaptic transmission was chronically blocked by $20 \mu \mathrm{M}$ BIC, AMPAR-mediated responses in the infected cell were 2-fold higher than in neighboring control neurons (Fig. 4.13A; AMPAR EPSCs control: $-44.3 \pm 3.7 \mathrm{pA}$, infected: $-93.2 \pm 13.1 \mathrm{pA}$ ). NMDAR EPSCs were not significantly different from the control neurons (NMDAR EPSCs control: $15.4 \pm 1.5$, infected: $22.7 \pm 4.6 \mathrm{pA}$ ), although there was a trend towards an increase (see Fig. 4.10A and 4.13C).

In the case of overexpressing PSD-93 $\epsilon$ while blocking inhibitory transmission, a similar 2.5-fold increase in AMPAR EPSCs was observed (Fig. 4.13B; AMPAR EPSCs control: $-35.4 \pm 3.0 \mathrm{pA}$, infected: $-90.0 \pm 9.0 \mathrm{pA})$, which is highly significant $(\mathrm{p}<0.001)$. In the case without activity manipulations, replacement of PSD-95 with PSD-93 $\epsilon$ resulted in a 1.8-fold increase in AMPAR EPSCs (Fig. 4.10B). Although there is a strong trend that an increase in excitatory activity leads to a further enhancement of AMPAR EPSC amplitude when PSD-95 is replaced with PSD-93e, the two sample populations do not differ significantly ( $\mathrm{p}=0.17$; Fig. 4.13C). NMDAR EPSCs are significantly increased by $43 \%$ in the infected neuron compared to the corresponding control cell (NMDAR

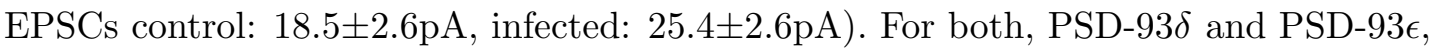


A $\quad \operatorname{sh} 95 p+$ PSD-93ß

$+\mathrm{BIC}$

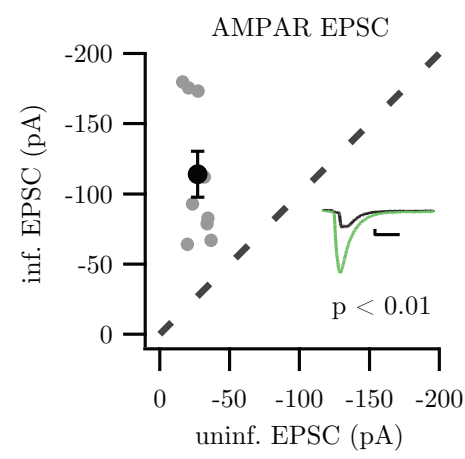

B $\quad \operatorname{sh} 95 p+$ PSD-93 $\beta$

+2 days $\mathrm{BIC}$

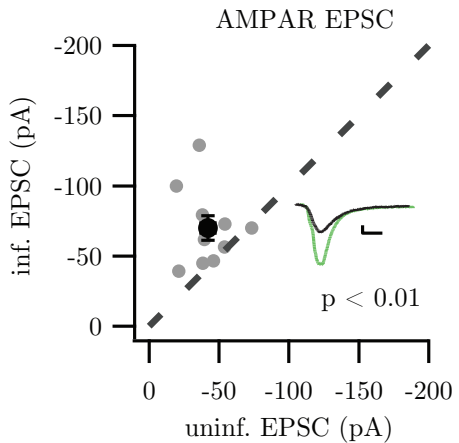

C $\quad \operatorname{sh} 95 p+$ PSD-93 $\beta$

$+\mathrm{BIC}+2$ days $\mathrm{AP}-\mathrm{V}$

AMPAR EPSC

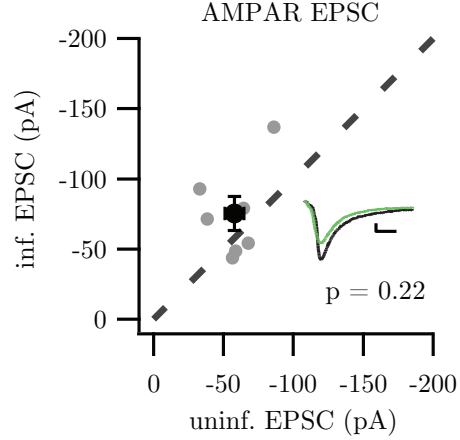

$\mathrm{D}$

AMPAR EPSC

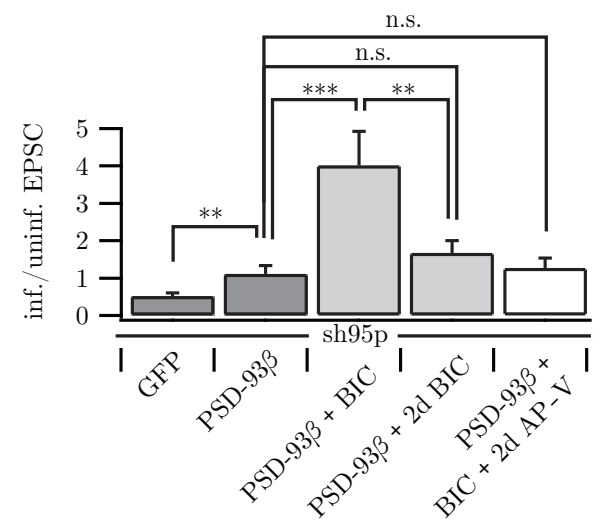

NMDAR EPSC

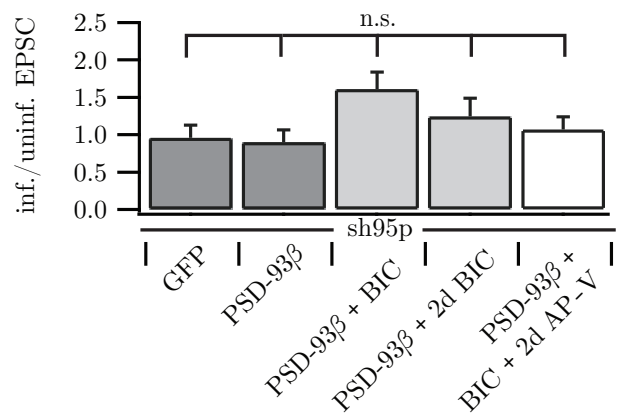

Figure 4.12: Activity-dependent increase of AMPAR EPSCs when overexpressing PSD-93 $\beta$ is NMDAR-dependent. Knockdown of endogenous PSD-95 with sh95p combined with overexpression of PSD-93 $\beta$. (A) Scatter plot of AMPAR EPSCs of control versus infected cell when incubating the slice culture in $20 \mu \mathrm{M}$ BIC from the day of infection on. Sample traces of infected (green) and control cell (black). (B) Scatter plot of AMPAR EPSCs of control versus infected cell when incubating the slice culture in $20 \mu \mathrm{M}$ BIC for 2 days before the recording. Sample traces of infected (green) and control cell (black). (C) Scatter plot of AMPAR EPSCs of control versus infected cell when incubating the slice culture in $20 \mu \mathrm{M}$ BIC from the day of infection on, and adding $50 \mu \mathrm{M} \mathrm{AP-V}$ for the last 2 days before the recording. Sample traces of infected (green) and control cell (black). (D) Summary of the effects of the activity manipulations when PSD-95 is replaced with PSD-93 $\beta$. Left: AMPAR EPSCs, Right: NMDAR EPSCs. Significance between pairs was tested with Wilcoxon Rank Sum test and between groups with Kruskal-Wallis test statistic. n.s. $\mathrm{p} \geq 0.05, * * \mathrm{p}<0.01,{ }^{* * *} \mathrm{p}<0.001$. Scale bars $10 \mathrm{pA}, 10 \mathrm{msec}$. 


\section{THE ROLE OF PSD-93 IN BASAL SYNAPTIC TRANSMISSION}

A $\operatorname{sh} 95 p+$ PSD-93 $\delta$

$+\mathrm{BIC}$
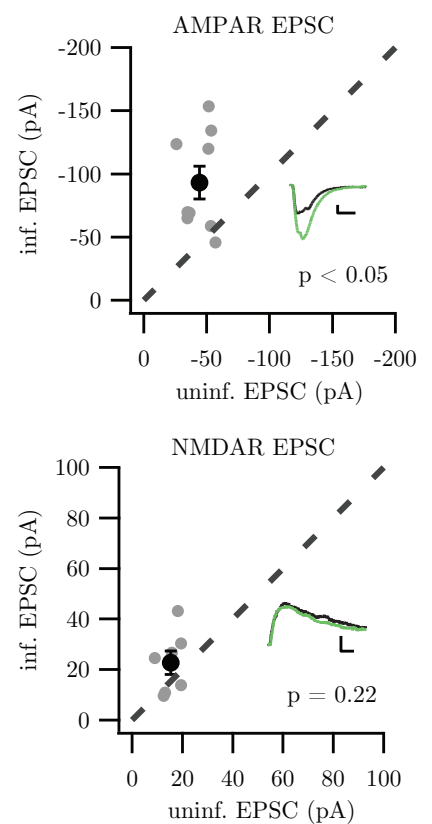

B sh95p + PSD-93є

$+\mathrm{BIC}$

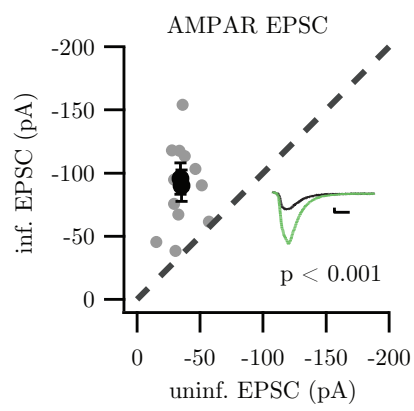

NMDAR EPSC

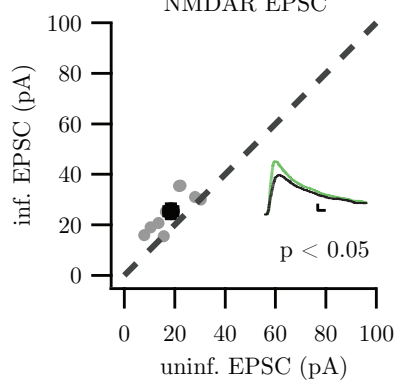

$\mathrm{C}$

AMPAR EPSC

$\operatorname{sh} 95 \mathrm{p}+\quad \operatorname{sh} 95 \mathrm{p}+$ PSD-938 PSD-93e

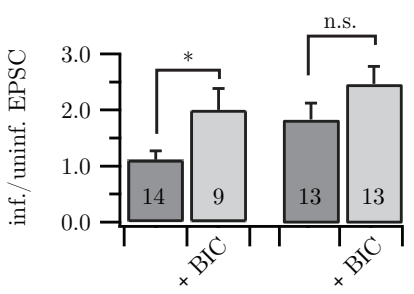

NMDAR EPSC

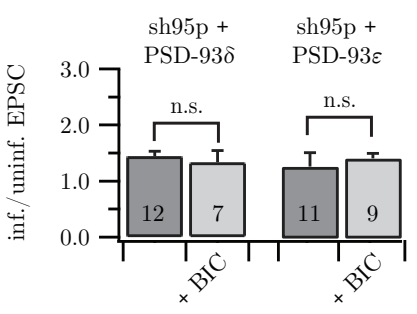

Figure 4.13: PSD-93 $\delta$ and possibly PSD-93 $\epsilon$ are affected by activity manipulations in the culture dish. The slice cultures were maintained in $20 \mu \mathrm{M}$ BIC from the day of infection on. (A) Scatter plot of AMPAR EPSCs (top, N=9) and NMDAR EPSCs (bottom, $\mathrm{N}=7$ ) of cells expressing sh95p and PSD-93 $\delta$ versus neighboring control neurons. Sample traces of infected (green) and control cell (black). (B) Scatter plot of AMPAR EPSCs (top, N=13) and NMDAR EPSCs (bottom, N=9) of cells expressing sh95p and PSD-93€ versus neighboring control neurons. Sample traces of infected (green) and control cell (black). (C) Summary of the effect of $20 \mu \mathrm{M}$ BIC on AMPAR (top) and NMDAR (bottom) EPSCs, when overexpressing PSD-93 $\delta$ and PSD-93 $\epsilon$ while endogenous PSD-95 is knocked down. n.s. $\mathrm{p} \geq 0.05, * \mathrm{p}<0.05$. Scale bars $10 \mathrm{pA}, 10 \mathrm{msec}$.

it remains to be tested whether the activity dependence of these isoforms requires NMDA receptor activation.

Thus, in addition to the L27 domain-containing isoform PSD-93 $\beta$, for which activity dependence was expected due to a previous study (Schlüter et al. 2006), PSD-93 $\delta$ and, to a smaller degree, PSD-93€ are regulated by activity. 
5

\section{Discussion}

As outlined in the introduction, both the identity of the PSD-93 isoforms expressed in the hippocampus as well as their functional properties have not been extensively studied. In fact, the physiological data available so far is quite contradictory (Carlisle et al. 2008; Elias et al. 2006; McGee et al. 2001; Tao et al. 2003). Despite extensive sequence similarity among the PSD-MAGUKs, there is evidence that they fulfill different functions. PSD-93 and PSD-95 have been suggested to exert opposing roles in plasticity (Carlisle et al. 2008), but identical roles in basal synaptic transmission (Elias et al. 2006). A recent study has shown that PSD-95 can act both as a structural scaffold to gate AMPARs as well as a signaling scaffold for proteins involved in LTD (Xu et al. 2008). A similar scenario could hold true for PSD-93, acting as a gate for AMPARs in basal synaptic transmission, and as a signaling scaffold for LTP. Nonetheless, it seems unlikely that in a single neuron two proteins have exactly the same function with regard to basal synaptic transmission.

Since the N-terminus of the PSD-MAGUKs largely controls their functional profile (Schlüter et al. 2006), and since PSD-93 possesses six alternative N-termini, identifying the isoforms of PSD-93 which are expressed in the hippocampus provides a key step in elucidating the protein's role.

In this thesis, I determined which isoforms are present in the hippocampus and analyzed their functional properties with respect to basal synaptic transmission and activity manipulations. Interestingly, all isoforms can rescue the reduction in AMPARmediated transmission when PSD-95 is knocked down by RNAi, but otherwise they exhibit distinct functional characteristics. 


\section{DISCUSSION}

\subsection{Relative abundance of PSD-93 isoforms in the hip- pocampus}

The $\alpha$-isoforms are the most abundant isoforms of PSD-93 in the hippocampus. Still, PSD-93 $\alpha_{1}$ and $\alpha_{2}$ were not detected by mass spectrometry. Since only a small number of unique peptides is theoretically available for PSD-93 $\alpha_{1}$ or PSD-93 $\alpha_{2}$, it is likely, that these peptide masses were not measured because the peptides did not fly well. Nonetheless, one peptide was observed which is predictive of both $\alpha$-isoforms of PSD93 but cannot distinguish between them (see Table 3.2, T35), and another peptide which indicates the presence of PSD-93 $\alpha_{1}, \alpha_{2}$, and/or $\epsilon$ (see Table 3.3, C23).

From the western blots it was inferred that the $\alpha$-isoforms of PSD-93 account for approximately $50 \%$ of all PSD-93 in the hippocampus (Fig. 3.14A). The $\alpha$-isoforms enrich relatively more in the P2 fraction compared to the remaining isoforms, and in addition are not solubilized well with $1 \%$ sodium N-lauryl sarcosine. This might indicate a different location of the $\alpha$ - isoforms, for instance in the core of the PSD.

Assuming that the lower two bands of PSD-93 in Figure 3.14A represent PSD-93 $\gamma_{1}$ and PSD-93e, respectively, they each account for 20\% of PSD-93 in the hippocampus. It has to be kept in mind that the presence of PSD-93 $\epsilon$ has not been proven and that this band might reflect a breakdown product of PSD-93 $\gamma_{1}$. PSD-93 $\delta$ makes up at most $5 \%$ of PSD-93, and PSD-93 $\beta$ is also not very abundant and reflects up to $10 \%$ of all PSD-93.

In addition, the relative abundance of PSD-93 with respect to the other PSDMAGUKs provides important information. With the help of antibodies and GFPfusion proteins for their normalization, I could demonstrate that in the hippocampus the amount of PSD-93 is about 42\% of PSD-95 (Fig. 3.14A). In contrast, Cheng et al. (2006) showed by absolute quantitative mass spectrometry that PSD-95 is six times more abundant than PSD-93 in the PSD fraction of rat forebrain. As discussed above, it is conceivable that only the $\alpha$-isoforms of PSD-93 are particularly enriched in the PSD fraction. Therefore, my results showing that the levels of PSD-93 $\alpha_{1 / 2}$ are about $20 \%$ of PSD-95 (Fig. 3.14B) are consistent with the 17\% predicted by Cheng et al. (2006). Another study showed by semi-quantitative mass spectrometry that both PSD-93 and SAP102 are about half of PSD-95 levels in the PSD fraction of rat forebrain (Peng et al. 2004). Since labeled recombinant PSD-95 but not PSD-93 was included in the 
analysis, the amount of PSD-93 could only be estimated from the number of peptides found for PSD-93, and is thus prone to inaccuracies.

Several methods can be used for quantitative protein profiling, such as quantitative immunofluorescence, western blotting, and mass spectrometry. Each of them has its own advantages and disadvantages, which will be discussed below. To study synaptic proteins, it is common to prepare a PSD fraction and limit the analysis to the proteins present in this fraction. However, when biochemically purified preparations are used for determining the abundance of certain proteins, it is possible, that the extraction steps enrich some proteins more than others, or that proteins originally present in other cellular compartments co-purify because of identical density and solubilization characteristics.

For absolute quantitative mass spectrometry, a known amount of labeled internal standard is added to the sample (e.g. the PSD fraction) before mass spectrometry. Several approaches can be taken, from addition of a labeled synthetic peptide as in the Absolute Quantification (AQUA, Gerber et al. 2003) to a labeled full-length protein (Brun et al. 2007). The AQUA approach as taken in Peng et al. (2004) and Cheng et al. (2006) may lead to an underestimation of the analyte if the protein under investigation is not digested completely or if it does not recover well from SDS-PAGE (Kito and Ito 2008). The intact labeled protein is expensive, but provides the highest accuracy. As it can only be added to the sample after protein extraction, this method has the same drawback as the AQUA technique since the extracted but not necessarily the total protein in the tissue is quantified (Kito and Ito 2008).

Quantitative immunofluorescence aims at circumventing the problem of biochemically purifying the PSD fraction and instead concentrates locally on individual synapses. The protein of interest is expressed recombinantly as a GFP-fusion protein and its abundance is determined using beads that are coated with a known number of GFP molecules. Using antibody staining, the relative amount of the endogenous protein with respect to the recombinant protein is calculated, thereby determining the absolute amount of native protein (Chiu et al. 2001; Okabe 2007). One caveat of this approach is that the expression of the recombinant protein might alter the abundance and/or localization of the native protein. In addition, photobleaching of GFP or background staining of the antibody might hamper the analysis. Also, this approach does not 


\section{DISCUSSION}

allow to differentiate between different isoforms of one protein unless isoform-specific antibodies are available.

Quantitative western blotting can distinguish between different isoforms of a protein, provided that they differ in their molecular weight. Hence, for studying PSD-93 isoforms, this method has proven useful. Again, the antibody for the protein of interest is normalized using recombinant GFP-fusion proteins and an $\alpha$-GFP antibody. One constrain of quantitative western blotting is the quality of the antibodies used. It is essential that the antibody signals are in their linear range.

From this discussion it follows that the "gold standard" method for quantitative protein profiling has yet to be developed. Still, the methods presented can in principle produce accurate estimates of the amount of protein present, but the results have to be carefully interpreted.

\subsection{The PSD-93 $\alpha$-isoforms behave $\alpha$-atypical}

In the study of Schlüter et al. (2006), it was shown that the $\alpha$-isoforms of PSD-95 and SAP97 are stunningly alike. Both SAP97 $\alpha$ and PSD-95 $\alpha$, when overexpressed, lead to an increase in AMPAR EPSCs. Important to note, SAP97 $\alpha$ is rare in the hippocampus when compared to PSD-95 $\alpha$ levels.

PSD-93 exists mainly as the $\alpha$-isoform in the hippocampus. PSD-93 $\alpha_{1}$ overexpression has been suggested to lead to a similar increase in AMPAR EPSCs as PSD-95 $\alpha$ (Elias et al. 2006). In my hands, neither the overexpression of PSD-93 $\alpha_{1}$ nor PSD$93 \alpha_{2}$, with or without GFP attached to its C-terminus, led to an increase in AMPAR EPSCs. There are three lines of evidence supporting this experimental result:

- It has been previously observed that unphysiological overexpression can lead to effects that do not reflect the endogenous function of the protein under investigation. Biolistic transfection compared to lentiviral-mediated delivery in general results in much stronger expression levels of the transgene. For biolistic transfection, the expression levels also highly depend on the amount of plasmid per gold particle.

In the study of Nakagawa et al. (2004), biolistic transfection was used to overexpress PSD-95 $\alpha$. It led to a 7-8 fold increase of AMPAR EPSCs, and to a 1.8-fold increase with SAP97 $\beta$ overexpression. In contrast, other studies usually 
observed a 2- to 3-fold increase when PSD-95 $\alpha$ was overexpressed (Elias et al. 2006; Schlüter et al. 2006; Schnell et al. 2002). For SAP97 $\beta$ overexpression no alterations of AMPAR EPSCs are detected (Ehrlich and Malinow 2004; Schlüter et al. 2006; Schnell et al. 2002), and one study even observed a decrease (Waites et al. 2009). Although both Elias et al. (2006) and Schnell et al. (2002) used biolistic transfection to overexpress PSD-95 $\alpha$, the divergence from Nakagawa et al. (2004) might be due to different amounts of plasmid coated around the gold particle, and hence different expression levels of the transgene.

Although Elias et al. (2006) studied both PSD-95 $\alpha$ and PSD-93 $\alpha_{1}$ using biolistic transfection, it has to be noted that the endogenous levels of PSD-93 and PSD95 differ (PSD-93 $\alpha_{1 / 2}$ makes up only $20 \%$ of PSD-95 $\alpha$ ). It is therefore possible that in case of PSD-93 $\alpha_{1}$ an unphysiological overexpression of the protein occurs. Hence, the observed phenotype might not reflect the endogenous function of this isoform. In contrast, lentiviral-mediated delivery of PSD-93 as in the present study only results in moderate expression levels.

- I could demonstrate that the deficiency of PSD-93 $\alpha_{1 / 2}$ to increase AMPAR EPSCs is due to an inhibitory domain inside the PSD-93 N-terminus. Likely, this repressive phenotype is overcome by high expression levels. Although exon 1 of PSD$93 \alpha_{2}$ and PSD-95 $\alpha$ in principle behave alike, the full N-terminus of PSD-95 was required to convert PSD-93 into an $\alpha$-isoform. This is in analogy to the study of Schnell et al. (2002), where placing the N-terminus of PSD-95 onto the SAP97 backbone caused the same phenotype.

As can be seen in Figure 3.9, exon 2 of PSD-95 is much shorter than those of PSD-93 and SAP97. From the experiments shown in Figure 4.7 and 4.8, it appears that exon 2 contains a domain which prevents PSD-93 $\alpha_{1 / 2}$ from affecting AMPAR-mediated currents. Exon 3 is quite conserved among the MAGUKs (Fig. 3.9), and I could show that exclusion of this exon from PSD-93 $\alpha_{2}$ does not change its inefficiency to increase AMPAR EPSCs (Fig. 4.6). The sequence ELVHVSEKNLSQIENVHGYVLQSHISPLK in exon 2 occurs with minor modifications also in SAP97. It is not clear why in case of SAP97 $\alpha$ overexpression AMPAR EPSCs are still increased. Either a C-terminal feature of SAP97, such as the I3 or I5 insert, overwrites the inhibitory action of exon 2, or single amino acids in exon 2 


\section{DISCUSSION}

are responsible for this effect. In exon 2 there are two serine residues in the motif xSHISP (arrows in Fig. 3.9). Using the web tool NetPhos 2.0, these residues were predicted to be phosphorylated in SAP97 $\alpha$ but not in PSD-93 $\alpha_{2}$ (SAP97 $\alpha$ S59: 0.778, S62: 0.585; PSD-93 $\alpha_{2}$ S69: 0.229, S72: 0.265; range 0-1, 0.5 being the threshold). It is conceivable that phosphorylation of these residues is required for SAP97 $\alpha$ to enhance AMPAR EPSCs, but this remains to be experimentally tested.

- Both $\alpha$-isoforms of PSD-93 can interact with a mutant of PSD-95 that only possesses the first two PDZ domains (Fig. 3.15C). If PSD-93 and PSD-95 fulfill similar functions, one would expect that PSD-93 $\alpha_{1 / 2}$ can translocate the mutant into the synapse. However, as shown in the study of Xu et al. (2008), in the absence of endogenous PSD-95, expression of the mutant construct does not rescue the impaired basal synaptic transmission whereas overexpression of the mutant construct increases AMPAR EPSCs. This indicates that full-length PSD-95 is required for this effect, and PSD-93 cannot compensate. One possibility is that PSD-93 and PSD-95 are localized to different sets of synapses as suggested by Elias et al. (2006), hence the observed association in Figure 3.15 is an experimental artifact, or occurs in the biosynthetic pathway before the protein's delivery to the synapse. On the other hand, immunogold labeling of PSD-93 and PSD95 showed the two proteins in the same spine (Degiorgis et al. 2008), and their colocalization is estimated to be $33 \%$ (Sans et al. 2000).

Instead of PSD-93 $\alpha_{1 / 2}$, PSD-93 $\epsilon$ behaves like a classical $\alpha$-isoform as it increases AMPAR EPSCs two-fold. Interestingly, it does not interact N-terminally with PSD-95 as could be appreciated in Figure 3.15B. Also, it does not appear to multimerize with itself as demonstrated using HEK293T cells (data not shown). Hence, in contrast to PSD-95 which needs homomultimerization for proper synaptic function (Schlüter et al. 2006), PSD-93 $\epsilon$ increases AMPAR EPSCs by another yet unknown mechanism.

\subsection{PSD-93 $\delta$ affects a conductance other than NMDARs}

PSD-93 not only leads to an increase in AMPAR EPSCs, but also to an increase $60 \mathrm{msec}$ after the peak when the cell is clamped at $+40 \mathrm{mV}$ (Fig. $4.4 \mathrm{C}$ ). This increase is also 
observed when overexpressing PSD-95 $\alpha$ (Fig. 4.8A). In a detailed pharmacological analysis, I could demonstrate that the increased current observed at $+40 \mathrm{mV}$ is neither due to AMPAR-mediated current, nor to current through NMDARs or KARs (Fig. 4.5C, D). Besides, there is no change in the presynaptic release probability (Fig. 4.5A).

CA1 pyramidal neurons are equipped with a variety of voltage-gated conductances. Voltage-dependent $\mathrm{Na}^{+}$and $\mathrm{Ca}^{2+}$ as well as A-type $\mathrm{K}^{+}$channels are distributed along the dendrite and fulfill functions in action potential backpropagation and control of membrane excitability (Andersen et al. 2007).

PSD-93 $\delta$ has been shown to cluster the inward rectifier $\mathrm{K}^{+}$channel Kir2.1, although no physiological effect of the association has been observed (Leyland and Dart 2004). Since Kir channels are blocked at positive membrane potentials (Doupnik et al. 1995), this association cannot be responsible for the observed effects of PSD-93 $\delta$ overexpression at $+40 \mathrm{mV}$.

Small conductance $\mathrm{Ca}^{2+}$-activated potassium channels (SK channels) can shunt EPSCs, although traditionally they have been shown to mediate the afterhyperpolarization current. A changed distribution of SK channels in response to PSD-93 $\delta$ overexpression, however, cannot account for the increase in transmission observed at $+40 \mathrm{mV}$. If the number of SK channels was reduced, one would expect less shunting. SK channels are activated by $\mathrm{Ca}^{2+}$ influx through NMDARs (Ngo-Anh et al. 2005). Therefore, even in the presence of NBQX, the reduction in shunting and thus enhanced EPSCs should be observed. This is not the case as can be seen in Figure 4.5C, hence making it unlikely that SK channels are responsible for the increase in conductance. An experimental approach to directly examine the role of SK channels would be to record in the presence of bicuculline since in addition to being an antagonist of $\mathrm{GABA}_{\mathrm{A}}$ receptors, it blocks SK channels.

In hippocampal CA1 pyramidal neurons it has been shown that activation of $\mathrm{Na}^{+}$ and $\mathrm{Ca}^{2+}$ channels can increase the size of subthreshold EPSPs measured in the soma, and prolong its duration (Magee and Johnston 1995). The drug QX-314, which is commonly used in intracellular solutions to block $\mathrm{Na}^{+}$channels (Strichartz 1973), has been shown to also influence $\mathrm{Ca}^{2+}$ currents (Talbot and Sayer 1996) and G proteincoupled inwardly-rectifying potassium channels (GIRKs, Andrade 1991). Intriguingly, whereas Schlüter et al. (2006) included QX-314 in their internal solution and did not observe an increased conductance when overexpressing PSD-95 $\alpha$ and clamping the cell 


\section{DISCUSSION}

at $+40 \mathrm{mV}$, I did not include QX-314 in the internal solution and observed an increase in the conductance at $+40 \mathrm{mV}$. However, a contribution of $\mathrm{Na}^{+}$and $\mathrm{Ca}^{2+}$ channels seems unlikely. Both $\mathrm{Na}^{+}$and $\mathrm{Ca}^{2+}$ channels are known to inactivate very fast and therefore should be inactive when the cell is voltage-clamped at $+40 \mathrm{mV}$ (reviewed in Goldin 2003; Hering et al. 2000). GIRKs are blocked at positive membrane potentials (Doupnik et al. 1995), and hence cannot contribute to the conductance observed.

Thus, it remains elusive where the increase in conductance results from when PSD$93 \delta$ is overexpressed and the cell is clamped at $+40 \mathrm{mV}$.

\subsection{PSD-93 has no role in basal synaptic transmission}

Half of PSD-93 in the hippocampus is made up of the $\alpha$-isoforms. However, the $\alpha$ isoforms behave $\alpha$-atypical, as they do not increase AMPAR EPSCs when overexpressed (Fig. 4.3). The only isoforms which affect AMPAR EPSCs when overexpressed are PSD-93 $\delta$ and PSD-93 $\epsilon$. Merely 5\% of PSD-93 in the hippocampus is PSD-93 $\delta$, and the existence of PSD-93 $\epsilon$ in the hippocampus is still ambiguous. Assuming that the lowest band on the western blots is PSD-93 $\epsilon$, it makes up about 20\% of total PSD-93. Taking PSD-93 $\delta$ and $\epsilon$ together, they reflect $10 \%$ of PSD-95 levels. It is therefore plausible that the knockdown of PSD-93 does not lead to the same 50\% reduction of AMPAR EPSCs as does PSD-95 knockdown. This is in contrast to the study of Elias et al. (2006), in which a 50\% reduction of AMPAR EPSCs was observed when PSD-93 was knocked down. Consistent with my results, none of the groups studying the effect of the genetic knockout of PSD-93 noticed a change in basal synaptic transmission (Carlisle et al. 2008; Elias et al. 2006; Tao et al. 2003). Contrarily, some groups observed an effect on parameters of basal synaptic transmission in PSD-95 knockout animals (Béïque et al. 2006; Carlisle et al. 2008; but see Migaud et al. 1998), in line with the RNAi-mediated knockdown of PSD-95 which resulted in a 50\% reduction of AMPAR EPSCs (Elias et al. 2006; Nakagawa et al. 2004; Schlüter et al. 2006). Hence, although it still remains unclear why my results differ from those of Elias et al. (2006), the present study is consistent and is in agreement with the results found in the PSD-93 KO mice. 


\subsection{Activity dependence is not specific for the L27 domain}

PSD-93 $\beta$ behaves like a bona fide $\beta$-isoform, as it shows a strong increase in AMPAR EPSCs when the excitatory activity in the culture dish is elevated (Fig. 4.12). The increase is twice as strong as for SAP97 $\beta$ (Schlüter et al. 2006), and as with SAP97 $\beta$, the effect can be blocked with the NMDAR antagonist AP-V (Fig. 4.12C). The increase of AMPAR EPSCs is not as strong if bicuculline is added to the culture medium only for two rather than five to seven days. Using dissociated cultures Ehlers (2003) showed that activity manipulations usually result in short-term effects, peaking 48-72 hours after the start of the manipulation and rapidly returning to baseline levels. On the other hand, whereas endogenous synaptic PSD-95 and SAP102 levels have been shown to be affected by treatment with tetrodotoxin or bicuculline, PSD-93 levels remained constant (Ehlers 2003). Most likely, the overexpression of PSD-93 $\beta$ paired with activity leads to a signaling cascade that increases the number of functional AMPARs at the synapse, without an absolute increase in PSD-93 $\beta$ protein levels.

Importantly, not only PSD-93 $\beta$ showed activity-dependence, but also PSD-938. When PSD-93 $\delta$ was overexpressed and the excitatory activity in the culture was raised with bicuculline, AMPAR EPSCs were increased two-fold, without affecting NMDAR EPSCs. PSD-93 $\epsilon$ also showed an increase of AMPAR EPSCs when the excitatory activity in the culture was high. However, since AMPAR EPSCs were already 1.8-fold increased when PSD-93 $\epsilon$ was overexpressed or when PSD-95 was replaced with PSD$93 \epsilon$, this additional increase of $37 \%$ did not reach statistical significance and hence only reflects a trend.

So far, the activity-dependence has been attributed to the L27 domain of the $\beta$ isoforms (Schlüter et al. 2006). Since PSD-93 $\delta$ does not possess an L27 domain, there might be a different feature conferring activity dependence. Moreover, it can be ruled out that being non- $\alpha$ is sufficient for possessing activity-dependence, as PSD-93 $\gamma_{1}$ does not show activity dependent behavior.

\subsection{Conclusions and Outlook}

The present study demonstrates that the N-terminal isoforms of PSD-93 define the protein's function and make each of them unique. This is summarized in Table 5.1. Importantly, the $\alpha$-isoforms of PSD-93 do not behave like classical $\alpha$-isoforms. Since 


\section{DISCUSSION}

Table 5.1: Summary of the expression and function of PSD-93 isoforms (Hp, hippocampus; KD, knockdown; $\rightarrow$, no change; $\nearrow$, slight increase; $\uparrow$ significant increase).

\begin{tabular}{cccccc}
\hline PSD-93 & $\begin{array}{c}\text { Expressed } \\
\text { Isoform }\end{array}$ & $\begin{array}{c}\text { Abundance } \\
\text { in } \mathrm{Hp}\end{array}$ & $\begin{array}{c}\text { AMPAR } \\
\text { in }\end{array}$ & $\begin{array}{c}\text { Rescue of } \\
\text { PSD-95 KD }\end{array}$ & $\begin{array}{c}\text { Activity } \\
\text { dependence }\end{array}$ \\
\hline$\alpha_{1 / 2}$ & $\checkmark$ & $\sim 50 \%$ & $\rightarrow$ & $\checkmark$ & $\rightarrow$ \\
$\beta$ & $\checkmark$ & $\sim 10 \%$ & $\rightarrow$ & $\checkmark$ & $\uparrow$ \\
$\gamma_{1}$ & $\checkmark$ & $\sim 20 \%$ & $\rightarrow$ & $\checkmark$ & $\rightarrow$ \\
$\delta$ & $\checkmark$ & $<5 \%$ & $\uparrow$ & $\checkmark$ & $\uparrow$ \\
$\epsilon$ & $?$ & $?$ & $\uparrow$ & $\checkmark$ & $\nearrow$ \\
\hline
\end{tabular}

PSD-93 mainly occurs as the $\alpha$-isoform in the hippocampus, this is consistent with the fact that basal synaptic transmission is not altered when PSD-93 is knocked down by RNA interference.

Showing the diversity of the different isoforms sets the framework for follow-up studies. There are still important questions to target:

- Is PSD-93 palmitoylated at C33 and C35? Is the palmitoylation required to lead to the increased conductance at $+40 \mathrm{mV}$ ? What is the nature of this conductance; is it sensitive to QX-314 in the intracellular solution in the recording pipette?

- Does the C-terminal splicing, thus the incorporation of the I3- and/or I5-like inserts, change the protein's function? For SAP97 specific protein interactions have been attributed to the I3 and/or I5 exons. The interaction with protein $4.1 \mathrm{~N}$ via the I3 insert has been described by Lue et al. (1994) and was suggested to be crucial for synaptic targeting of SAP97 (Rumbaugh et al. 2003). The combination of the exons I3 and I5 is thought to be important for a CaMKII $\alpha$ modulated binding of AKAP79/150 to SAP97 (Nikandrova et al. 2010). Which N-terminal isoforms of PSD-93 pair with which C-terminal isoforms?

- Having shown that the activity-dependence does not require an L27 domain, it remains to be elucidated where the activity domain is localized in the protein. Do the activity-dependent isoforms participate in LTP? 


\section{References}

H. Adesnik and R. A. Nicoll. Conservation of glutamate receptor 2-containing AMPA receptors during long-term potentiation. J Neurosci, 27(17):4598-4602, Apr 2007. Page 5.

D. G. Amaral and M. P. Witter. The three-dimensional organization of the hippocampal formation: a review of anatomical data. Neuroscience, 31(3):571-591, 1989. Page 2.

P. Andersen, T. V. Bliss, and K. K. Skrede. Lamellar organization of hippocampal excitatory pathways. Exp Brain Res, 13(2):222-238, 1971. Page 2.

P. Andersen, A. F. Soleng, and M. Raastad. The hippocampal lamella hypothesis revisited. Brain Res, 886(1-2):165-171, Dec 2000. Page 4.

P. Andersen, R. Morris, D. Amaral, T. Bliss, and J. O'Keefe, editors. The Hippocampus Book (Oxford Neuroscience Series). Oxford University Press, USA, 1 edition, 112007. ISBN 9780195100273. Page 2, 3, 97.

R. Andrade. Blockade of neurotransmitter-activated K+ conductance by QX-314 in the rat hippocampus. Eur J Pharmacol, 199(2):259-262, Jun 1991. Page 97.

P. Ascher and L. Nowak. The role of divalent cations in the N-methyl-D-aspartate responses of mouse central neurones in culture. J Physiol, 399:247-266, May 1988. Page 4.

J. L. Ashurst, C.-K. Chen, J. G. R. Gilbert, K. Jekosch, S. Keenan, P. Meidl, S. M. Searle, J. Stalker, R. Storey, S. Trevanion, L. Wilming, and T. Hubbard. The Vertebrate Genome Annotation (Vega) database. Nucleic Acids Res, 33(Database issue): D459-D465, Jan 2005. Page 34. 


\section{REFERENCES}

A. A. Bagal, J. P. Y. Kao, C. Tang, and S. M. Thompson. Long-term potentiation of exogenous glutamate responses at single dendritic spines. Proc Natl Acad Sci U S A, 102(40):14434-14439, Oct 2005. Page 5.

C. Bats, L. Groc, and D. Choquet. The interaction between Stargazin and PSD95 regulates AMPA receptor surface trafficking. Neuron, 53(5):719-734, Mar 2007. Page 9.

J.-C. Béique and R. Andrade. PSD-95 regulates synaptic transmission and plasticity in rat cerebral cortex. J Physiol, 546(Pt 3):859-867, Feb 2003. Page 9.

J.-C. Béïque, D.-T. Lin, M.-G. Kang, H. Aizawa, K. Takamiya, and R. L. Huganir. Synapse-specific regulation of AMPA receptor function by PSD-95. Proc Natl Acad Sci U S A, 103(51):19535-19540, Dec 2006. Page 8, 9, 11, 82, 98.

H. C. Birnboim and J. Doly. A rapid alkaline extraction procedure for screening recombinant plasmid DNA. Nucleic Acids Res, 7(6):1513-1523, Nov 1979. Page 15.

M. M. Blanchard, P. Taillon-Miller, P. Nowotny, and V. Nowotny. PCR buffer optimization with uniform temperature regimen to facilitate automation. PCR Methods Appl, 2(3):234-240, Feb 1993. Page 16.

T. V. Bliss and T. Lømo. Long-lasting potentiation of synaptic transmission in the dentate area of the anaesthetized rabbit following stimulation of the perforant path. J Physiol, 232(2):331-356, Jul 1973. Page 2.

N. Blom, S. Gammeltoft, and S. Brunak. Sequence and structure-based prediction of eukaryotic protein phosphorylation sites. J Mol Biol, 294(5):1351-1362, Dec 1999. Page 34 .

A. J. Borgdorff and D. Choquet. Regulation of AMPA receptor lateral movements. Nature, 417(6889):649-653, Jun 2002. Page 6.

D. S. Bredt and R. A. Nicoll. AMPA receptor trafficking at excitatory synapses. Neuron, 40(2):361-379, Oct 2003. Page 2, 5 .

J. E. Brenman, K. S. Christopherson, S. E. Craven, A. W. McGee, and D. S. Bredt. Cloning and characterization of postsynaptic density 93, a nitric oxide synthase interacting protein. J Neurosci, 16(23):7407-7415, Dec 1996. Page 8, 37, 39, 61. 
T. R. Brummelkamp, R. Bernards, and R. Agami. A system for stable expression of short interfering RNAs in mammalian cells. Science, 296(5567):550-553, Apr 2002. Page 13.

V. Brun, A. Dupuis, A. Adrait, M. Marcellin, D. Thomas, M. Court, F. Vandenesch, and J. Garin. Isotope-labeled protein standards: toward absolute quantitative proteomics. Mol Cell Proteomics, 6(12):2139-2149, Dec 2007. Page 93.

I. Bureau, S. Bischoff, S. F. Heinemann, and C. Mulle. Kainate receptor-mediated responses in the CA1 field of wild-type and GluR6-deficient mice. J Neurosci, 19(2): 653-663, Jan 1999. Page 77.

C. Cai, S. K. Coleman, K. Niemi, and K. Keinänen. Selective binding of synapseassociated protein 97 to GluR-A alpha-amino-5-hydroxy-3-methyl-4-isoxazole propionate receptor subunit is determined by a novel sequence motif. J Biol Chem, 277 (35):31484-31490, Aug 2002. Page 9.

H. J. Carlisle, A. E. Fink, S. G. N. Grant, and T. J. O'Dell. Opposing effects of PSD93 and PSD-95 on long-term potentiation and spike-timing dependent plasticity. $J$ Physiol, Oct 2008. Page 11, 69, 82, 91, 98.

P. E. Castillo, R. C. Malenka, and R. A. Nicoll. Kainate receptors mediate a slow postsynaptic current in hippocampal CA3 neurons. Nature, 388(6638):182-186, Jul 1997. Page 77.

L. Chen, D. M. Chetkovich, R. S. Petralia, N. T. Sweeney, Y. Kawasaki, R. J. Wenthold, D. S. Bredt, and R. A. Nicoll. Stargazin regulates synaptic targeting of AMPA receptors by two distinct mechanisms. Nature, 408(6815):936-943, 2000. Page 9.

D. Cheng, C. C. Hoogenraad, J. R., E. Ramm, M. A. Schlager, D. M. Duong, P. Xu, S. R. Wijayawardana, J. Hanfelt, T. Nakagawa, M. Sheng, and J. Peng. Relative and absolute quantification of postsynaptic density proteome isolated from rat forebrain and cerebellum. Mol Cell Proteomics, 5(6):1158-1170, Jun 2006. Page 92, 93.

D. M. Chetkovich, R. C. Bunn, S.-H. Kuo, Y. Kawasaki, M. Kohwi, and D. S. Bredt. Postsynaptic targeting of alternative postsynaptic density-95 isoforms by distinct mechanisms. J Neurosci, 22(15):6415-6425, Aug 2002. Page 7, 8, 9, 38. 


\section{REFERENCES}

C. S. Chiu, E. Kartalov, M. Unger, S. Quake, and H. A. Lester. Single-molecule measurements calibrate green fluorescent protein surface densities on transparent beads for use with 'knock-in' animals and other expression systems. J Neurosci Methods, 105(1):55-63, Jan 2001. Page 93.

P. Chomczynski and N. Sacchi. Single-step method of RNA isolation by acid guanidinium thiocyanate-phenol-chloroform extraction. Anal Biochem, 162(1):156-159, Apr 1987. Page 20.

G. L. Collingridge, J. T. R. Isaac, and Y. T. Wang. Receptor trafficking and synaptic plasticity. Nat Rev Neurosci, 5(12):952-962, Dec 2004. Page 5.

R. Cossart, J. Epsztein, R. Tyzio, H. Becq, J. Hirsch, Y. Ben-Ari, and V. Crépel. Quantal release of glutamate generates pure kainate and mixed AMPA/kainate EPSCs in hippocampal neurons. Neuron, 35(1):147-159, Jul 2002. Page 76.

S. E. Craven, A. E. El-Husseini, and D. S. Bredt. Synaptic targeting of the postsynaptic density protein PSD-95 mediated by lipid and protein motifs. Neuron, 22(3):497-509, Mar 1999. Page 7.

S. Dakoji, S. Tomita, S. Karimzadegan, R. A. Nicoll, and D. S. Bredt. Interaction of transmembrane AMPA receptor regulatory proteins with multiple membrane associated guanylate kinases. Neuropharmacology, 45(6):849-856, Nov 2003. Page 9.

J. Degiorgis, J. Galbraith, A. Dosemeci, X. Chen, and T. Reese. Distribution of the scaffolding proteins PSD-95, PSD-93, and SAP97 in isolated PSDs. Brain Cell Biol, Apr 2008. Page 96.

C. A. Doupnik, N. Davidson, and H. A. Lester. The inward rectifier potassium channel family. Curr Opin Neurobiol, 5(3):268-277, Jun 1995. Page 97, 98.

M. D. Ehlers. Activity level controls postsynaptic composition and signaling via the ubiquitin-proteasome system. Nat Neurosci, 6(3):231-242, Mar 2003. Page 99.

I. Ehrlich and R. Malinow. Postsynaptic density 95 controls AMPA receptor incorporation during long-term potentiation and experience-driven synaptic plasticity. $J$ Neurosci, 24(4):916-927, Jan 2004. Page 9, 10, 95. 
A. E. El-Husseini, S. E. Craven, D. M. Chetkovich, B. L. Firestein, E. Schnell, C. Aoki, and D. S. Bredt. Dual palmitoylation of PSD-95 mediates its vesiculotubular sorting, postsynaptic targeting, and ion channel clustering. J Cell Biol, 148(1):159-172, Jan 2000a. Page 7.

A. E. El-Husseini, E. Schnell, D. M. Chetkovich, R. A. Nicoll, and D. S. Bredt. PSD95 involvement in maturation of excitatory synapses. Science, 290(5495):1364-1368, Nov 2000b. Page 8.

A. E. El-Husseini, J. R. Topinka, J. E. Lehrer-Graiwer, B. L. Firestein, S. E. Craven, C. Aoki, and D. S. Bredt. Ion channel clustering by membrane-associated guanylate kinases. Differential regulation by N-terminal lipid and metal binding motifs. J Biol Chem, 275(31):23904-23910, Aug 2000c. Page 8, 37, 63.

A. E. El-Husseini, E. Schnell, S. Dakoji, N. Sweeney, Q. Zhou, O. Prange, C. GauthierCampbell, A. Aguilera-Moreno, R. A. Nicoll, and D. S. Bredt. Synaptic strength regulated by palmitate cycling on PSD-95. Cell, 108(6):849-863, Mar 2002. Page 7.

G. M. Elias, L. Funke, V. Stein, S. G. Grant, D. S. Bredt, and R. A. Nicoll. Synapsespecific and developmentally regulated targeting of AMPA receptors by a family of MAGUK scaffolding proteins. Neuron, 52(2):307-320, Oct 2006. Page 9, 10, 11, 44, $69,74,82,83,91,94,95,96,98$.

B. L. Firestein, S. E. Craven, and D. S. Bredt. Postsynaptic targeting of MAGUKs mediated by distinct N-terminal domains. Neuroreport, 11(16):3479-3484, Nov 2000. Page 8.

M. Frerking and R. A. Nicoll. Synaptic kainate receptors. Curr Opin Neurobiol, 10(3): 342-351, Jun 2000. Page 76.

T. F. Freund. Interneuron Diversity series: Rhythm and mood in perisomatic inhibition. Trends Neurosci, 26(9):489-495, Sep 2003. Page 2.

Y. Fukata and M. Fukata. Protein palmitoylation in neuronal development and synaptic plasticity. Nat Rev Neurosci, 11(3):161-175, Mar 2010. Page 7. 


\section{REFERENCES}

Y. Fukata, A. V. Tzingounis, J. C. Trinidad, M. Fukata, A. L. Burlingame, R. A. Nicoll, and D. S. Bredt. Molecular constituents of neuronal AMPA receptors. J Cell Biol, 169(3):399-404, May 2005. Page 9.

L. Funke. Function of the PDZ-domain containing protein familiy of PSD-MAGUKs in AMPA receptor targeting to excitatory synapses. $\mathrm{PhD}$ thesis, Freie Universität Berlin, Germany, April 2007. Page 35, 37.

K. Futai, M. J. Kim, T. Hashikawa, P. Scheiffele, M. Sheng, and Y. Hayashi. Retrograde modulation of presynaptic release probability through signaling mediated by PSD95-neuroligin. Nat Neurosci, 10(2):186-195, Feb 2007. Page 9, 74.

B. H. Gähwiler. Organotypic monolayer cultures of nervous tissue. J Neurosci Methods, 4(4):329-342, Dec 1981. Page 2.

S. A. Gerber, J. Rush, O. Stemman, M. W. Kirschner, and S. P. Gygi. Absolute quantification of proteins and phosphoproteins from cell lysates by tandem MS. Proc Natl Acad Sci U S A, 100(12):6940-6945, Jun 2003. Page 93.

A. L. Goldin. Mechanisms of sodium channel inactivation. Curr Opin Neurobiol, 13 (3):284-290, Jun 2003. Page 98.

F. L. Graham, J. Smiley, W. C. Russell, and R. Nairn. Characteristics of a human cell line transformed by DNA from human adenovirus type 5. J Gen Virol, 36(1):59-74, Jul 1977. Page 22.

N. K. Gray and M. Wickens. Control of translation initiation in animals. Annu Rev Cell Dev Biol, 14:399-458, 1998. Page 61.

Y. Hayashi, S. H. Shi, J. A. Esteban, A. Piccini, J. C. Poncer, and R. Malinow. Driving AMPA receptors into synapses by LTP and CaMKII: requirement for GluR1 and PDZ domain interaction. Science, 287(5461):2262-2267, Mar 2000. Page 5.

K. He, L. Song, L. W. Cummings, J. Goldman, R. L. Huganir, and H.-K. Lee. Stabilization of Ca2+-permeable AMPA receptors at perisynaptic sites by GluR1-S845 phosphorylation. Proc Natl Acad Sci U S A, 106(47):20033-20038, Nov 2009. Page 5. 
D. O. Hebb. The organization of behavior: a neuropsychological theory. Wiley, 1949. Page 2.

M. Heine, L. Groc, R. Frischknecht, J.-C. Béique, B. Lounis, G. Rumbaugh, R.L. Huganir, L. Cognet, and D. Choquet. Surface mobility of postsynaptic AMPARs tunes synaptic transmission. Science, 320(5873):201-205, Apr 2008. Page 6.

S. Hering, S. Berjukow, S. Sokolov, R. Marksteiner, R. G. Weiss, R. Kraus, and E. N. Timin. Molecular determinants of inactivation in voltage-gated $\mathrm{Ca} 2+$ channels. $J$ Physiol, 528 Pt 2:237-249, Oct 2000. Page 98.

M. D. Hoffman and J. Kast. Mass spectrometric characterization of lipid-modified peptides for the analysis of acylated proteins. J Mass Spectrom, 41(2):229-241, Feb 2006. Page 63.

Y. P. Hsueh, E. Kim, and M. Sheng. Disulfide-linked head-to-head multimerization in the mechanism of ion channel clustering by PSD-95. Neuron, 18(5):803-814, May 1997. Page 7.

J. E. Huettner and R. W. Baughman. Primary culture of identified neurons from the visual cortex of postnatal rats. J Neurosci, 6(10):3044-3060, Oct 1986. Page 23.

J. T. Isaac, R. A. Nicoll, and R. C. Malenka. Evidence for silent synapses: implications for the expression of LTP. Neuron, 15(2):427-434, Aug 1995. Page 10.

H. Kawabe, A. Neeb, K. Dimova, S. M. Young, M. Takeda, S. Katsurabayashi, M. Mitkovski, O. A. Malakhova, D.-E. Zhang, M. Umikawa, K. Kariya, S. Goebbels, K.-A. Nave, C. Rosenmund, O. Jahn, J. Rhee, and N. Brose. Regulation of Rap2A by the ubiquitin ligase Nedd4-1 controls neurite development. Neuron, 65(3):358-372, Feb 2010. Page 25, 26, 37, 66.

R. P. Kesner and R. O. Hopkins. Mnemonic functions of the hippocampus: a comparison between animals and humans. Biol Psychol, 73(1):3-18, Jul 2006. Page 2.

E. Kim and M. Sheng. PDZ domain proteins of synapses. Nat Rev Neurosci, 5(10): 771-781, Oct 2004. Page 6. 


\section{REFERENCES}

E. Kim, K. O. Cho, A. Rothschild, and M. Sheng. Heteromultimerization and NMDA receptor-clustering activity of Chapsyn-110, a member of the PSD-95 family of proteins. Neuron, 17(1):103-113, Jul 1996. Page 7, 55.

K. Kito and T. Ito. Mass spectrometry-based approaches toward absolute quantitative proteomics. Curr Genomics, 9(4):263-274, Jun 2008. Page 93.

M. Kozak. An analysis of 5'-noncoding sequences from 699 vertebrate messenger RNAs. Nucleic Acids Res, 15(20):8125-8148, Oct 1987. Page 18.

M. A. Larkin, G. Blackshields, N. P. Brown, R. Chenna, P. A. McGettigan, H. McWilliam, F. Valentin, I. M. Wallace, A. Wilm, R. Lopez, J. D. Thompson, T. J. Gibson, and D. G. Higgins. Clustal W and Clustal X version 2.0. Bioinformatics, 23(21):2947-2948, Nov 2007. Page 34.

B. Laube, H. Hirai, M. Sturgess, H. Betz, and J. Kuhse. Molecular determinants of agonist discrimination by NMDA receptor subunits: analysis of the glutamate binding site on the NR2B subunit. Neuron, 18(3):493-503, Mar 1997. Page 4.

D. J. Laurie, I. Bartke, R. Schoepfer, K. Naujoks, and P. H. Seeburg. Regional, developmental and interspecies expression of the four NMDAR2 subunits, examined using monoclonal antibodies. Brain Res Mol Brain Res, 51(1-2):23-32, Nov 1997. Page 4.

G. Lavezzari, J. McCallum, R. L., and K. W. Roche. Differential binding of the AP2 adaptor complex and PSD-95 to the C-terminus of the NMDA receptor subunit NR2B regulates surface expression. Neuropharmacology, 45(6):729-737, Nov 2003. Page 66.

S. Lee, S. Fan, O. Makarova, S. Straight, and B. Margolis. A novel and conserved protein-protein interaction domain of mammalian Lin-2/CASK binds and recruits SAP97 to the lateral surface of epithelia. Mol Cell Biol, 22(6):1778-1791, Mar 2002. Page 7.

A. S. Leonard, M. A. Davare, M. C. Horne, C. C. Garner, and J. W. Hell. SAP97 is associated with the alpha-amino-3-hydroxy-5-methylisoxazole-4-propionic acid receptor GluR1 subunit. J Biol Chem, 273(31):19518-19524, Jul 1998. Page 9. 
R. A. Lester, J. D. Clements, G. L. Westbrook, and C. E. Jahr. Channel kinetics determine the time course of NMDA receptor-mediated synaptic currents. Nature, 346(6284):565-567, Aug 1990. Page 4.

P. Lewis, M. Hensel, and M. Emerman. Human immunodeficiency virus infection of cells arrested in the cell cycle. EMBO J, 11(8):3053-3058, Aug 1992. Page 22.

M. L. Leyland and C. Dart. An alternatively spliced isoform of PSD-93/chapsyn 110 binds to the inwardly rectifying potassium channel, Kir2.1. J Biol Chem, 279(42): 43427-43436, Oct 2004. Page 25, 26, 39, 40, 53, 97.

X.-B. Liu, K. D. Murray, and E. G. Jones. Switching of NMDA receptor 2A and 2B subunits at thalamic and cortical synapses during early postnatal development. $J$ Neurosci, 24(40):8885-8895, Oct 2004. Page 4, 37.

C. Lois, E. J. Hong, S. Pease, E. J. Brown, and D. Baltimore. Germline transmission and tissue-specific expression of transgenes delivered by lentiviral vectors. Science, 295(5556):868-872, Feb 2002. Page 13, 17.

W. Lu, Y. Shi, A. C. Jackson, K. Bjorgan, M. J. During, R. Sprengel, P. H. Seeburg, and R. A. Nicoll. Subunit composition of synaptic AMPA receptors revealed by a single-cell genetic approach. Neuron, 62(2):254-268, Apr 2009. Page 5.

R. A. Lue, S. M. Marfatia, D. Branton, and A. H. Chishti. Cloning and characterization of hdlg: the human homologue of the Drosophila discs large tumor suppressor binds to protein 4.1. Proc Natl Acad Sci U S A, 91(21):9818-9822, Oct 1994. Page 40, 100.

K. S. Lundberg, D. D. Shoemaker, M. W. Adams, J. M. Short, J. A. Sorge, and E. J. Mathur. High-fidelity amplification using a thermostable DNA polymerase isolated from Pyrococcus furiosus. Gene, 108(1):1-6, Dec 1991. Page 16.

J. C. Magee and D. Johnston. Synaptic activation of voltage-gated channels in the dendrites of hippocampal pyramidal neurons. Science, 268(5208):301-304, Apr 1995. Page 97.

R. C. Malenka and M. F. Bear. LTP and LTD: an embarrassment of riches. Neuron, 44(1):5-21, Sep 2004. Page 2. 


\section{REFERENCES}

R. Malinow and R. C. Malenka. AMPA receptor trafficking and synaptic plasticity. Annu Rev Neurosci, 25:103-126, 2002. Page 2, 4, 5.

A. Marchler-Bauer and S. H. Bryant. CD-Search: protein domain annotations on the fly. Nucleic Acids Res, 32(Web Server issue):W327-W331, Jul 2004. Page 34.

J. R. Masters and G. N. Stacey. Changing medium and passaging cell lines. Nat Protoc, 2(9):2276-2284, 2007. Page 22.

C. J. McBain and A. Fisahn. Interneurons unbound. Nat Rev Neurosci, 2(1):11-23, Jan 2001. Page 2.

A. W. McGee, J. R. Topinka, K. Hashimoto, R. S. Petralia, S. Kakizawa, F. W. Kauer, A. Aguilera-Moreno, R. J. Wenthold, M. Kano, D. S. Bredt, and F. Kauer. PSD-93 knock-out mice reveal that neuronal MAGUKs are not required for development or function of parallel fiber synapses in cerebellum. J Neurosci, 21(9):3085-3091, May 2001. Page 10, 25, 69, 91.

M. Migaud, P. Charlesworth, M. Dempster, L. C. Webster, A. M. Watabe, M. Makhinson, Y. He, M. F. Ramsay, R. G. Morris, J. H. Morrison, T. J. O'Dell, and S. G. Grant. Enhanced long-term potentiation and impaired learning in mice with mutant postsynaptic density-95 protein. Nature, 396(6710):433-439, Dec 1998. Page 8, 9, $11,82,98$.

B. Milner and W. Penfield. The effect of hippocampal lesions on recent memory. Trans Am Neurol Assoc, (80th Meeting):42-48, 1955. Page 2.

B. Milner, L. R. Squire, and E. R. Kandel. Cognitive neuroscience and the study of memory. Neuron, 20(3):445-468, Mar 1998. Page 2.

J. Moffat, D. A. Grueneberg, X. Yang, S. Y. Kim, A. M. Kloepfer, G. Hinkle, B. Piqani, T. M. Eisenhaure, B. Luo, J. K. Grenier, A. E. Carpenter, S. Yin Foo, S. A. Stewart, B. R. Stockwell, N. Hacohen, W. C. Hahn, E. S. Lander, D. M. Sabatini, and D. E. Root. A lentiviral RNAi library for human and mouse genes applied to an arrayed viral high-content screen. Cell, 124(6):1283-1298, Mar 2006. Page 44. 
H. Monyer, N. Burnashev, D. J. Laurie, B. Sakmann, and P. H. Seeburg. Developmental and regional expression in the rat brain and functional properties of four NMDA receptors. Neuron, 12(3):529-540, Mar 1994. Page 4.

D. Muller, P. A. Buchs, and L. Stoppini. Time course of synaptic development in hippocampal organotypic cultures. Brain Res Dev Brain Res, 71(1):93-100, Jan 1993. Page 2.

S. Nada, T. Shima, H. Yanai, H. Husi, S. G. N. Grant, M. Okada, and T. Akiyama. Identification of PSD-93 as a substrate for the Src family tyrosine kinase Fyn. J Biol Chem, 278(48):47610-47621, Nov 2003. Page 8, 66.

T. Nakagawa, K. Futai, H. A. Lashuel, I. Lo, K. Okamoto, T. Walz, Y. Hayashi, and M. Sheng. Quaternary structure, protein dynamics, and synaptic function of SAP97 controlled by L27 domain interactions. Neuron, 44(3):453-467, Oct 2004. Page 8, 9, $74,82,94,95,98$.

G. Neves, S. F. Cooke, and T. V. P. Bliss. Synaptic plasticity, memory and the hippocampus: a neural network approach to causality. Nat Rev Neurosci, 9(1):65-75, Jan 2008. Page 68.

T. M. Newpher and M. D. Ehlers. Spine microdomains for postsynaptic signaling and plasticity. Trends Cell Biol, 19(5):218-227, May 2009. Page 6.

T. J. Ngo-Anh, B. L. Bloodgood, M. Lin, B. L. Sabatini, J. Maylie, and J. P. Adelman. SK channels and NMDA receptors form a Ca2+-mediated feedback loop in dendritic spines. Nat Neurosci, 8(5):642-649, May 2005. Page 97.

M. Niethammer, E. Kim, and M. Sheng. Interaction between the C terminus of NMDA receptor subunits and multiple members of the PSD-95 family of membraneassociated guanylate kinases. J Neurosci, 16(7):2157-2163, Apr 1996. Page 6.

Y. A. Nikandrova, Y. Jiao, A. J. Baucum, S. J. Tavalin, and R. J. Colbran. $\mathrm{Ca} 2+$ /calmodulin-dependent protein kinase II binds to and phosphorylates a specific SAP97 splice variant to disrupt association with AKAP79/150 and modulate alpha-amino-3-hydroxy-5-methyl-4-isoxazolepropionic acid-type glutamate receptor (AMPAR) activity. J Biol Chem, 285(2):923-934, Jan 2010. Page 42, 100. 


\section{REFERENCES}

Y. Ogawa, I. Horresh, J. S. Trimmer, D. S. Bredt, E. Peles, and M. N. Rasband. Postsynaptic density-93 clusters Kv1 channels at axon initial segments independently of Caspr2. J Neurosci, 28(22):5731-5739, May 2008. Page 44.

S. Okabe. Molecular anatomy of the postsynaptic density. Mol Cell Neurosci, 34(4): 503-518, Apr 2007. Page 93.

D. I. Papac, K. R. Thornburg, E. E. Bllesbach, R. K. Crouch, and D. R. Knapp. Palmitylation of a G-protein coupled receptor. Direct analysis by tandem mass spectrometry. J Biol Chem, 267(24):16889-16894, Aug 1992. Page 63.

M. Park, E. C. Penick, J. G. Edwards, J. A. Kauer, and M. D. Ehlers. Recycling endosomes supply AMPA receptors for LTP. Science, 305(5692):1972-1975, Sep 2004. Page 6 .

M. J. Parker, S. Zhao, D. S. Bredt, J. R. Sanes, and G. Feng. PSD93 regulates synaptic stability at neuronal cholinergic synapses. J Neurosci, 24(2):378-388, Jan 2004. Page 8, 17, 37, 38, 39, 40, 61.

M. Passafaro, V. Pich, and M. Sheng. Subunit-specific temporal and spatial patterns of AMPA receptor exocytosis in hippocampal neurons. Nat Neurosci, 4(9):917-926, Sep 2001. Page 5, 6.

J. Peng, M. Jong Kim, D. Cheng, D. M. Duong, S. P. Gygi, and M. Sheng. Semiquantitative proteomic analysis of rat forebrain postsynaptic density fractions by mass spectrometry. J Biol Chem, 279(20):21003-21011, May 2004. Page 92, 93.

D. N. Perkins, D. J. Pappin, D. M. Creasy, and J. S. Cottrell. Probability-based protein identification by searching sequence databases using mass spectrometry data. Electrophoresis, 20(18):3551-3567, Dec 1999. Page 31, 58.

K. Plant, K. A. Pelkey, Z. A. Bortolotto, D. Morita, A. Terashima, C. J. McBain, G. L. Collingridge, and J. T. R. Isaac. Transient incorporation of native GluR2-lacking AMPA receptors during hippocampal long-term potentiation. Nat Neurosci, 9(5): 602-604, May 2006. Page 5. 
K. Prybylowski, K. Chang, N. Sans, L. Kan, S. Vicini, and R. J. Wenthold. The synaptic localization of NR2B-containing NMDA receptors is controlled by interactions with PDZ proteins and AP-2. Neuron, 47(6):845-857, Sep 2005. Page 66.

R Development Core Team. R: A Language and Environment for Statistical Computing. R Foundation for Statistical Computing, Vienna, Austria, 2009. URL http://www . R-project.org. ISBN 3-900051-07-0. Page 34.

K. W. Roche and R. L. Huganir. Detection of protein phosphorylation in tissues and cells. Curr Protoc Neurosci, Chapter 5:Unit 5.14, May 2001. Page 66.

K. W. Roche, S. Standley, J. McCallum, C. Dune Ly, M. D. Ehlers, and R. J. Wenthold. Molecular determinants of NMDA receptor internalization. Nat Neurosci, 4(8):794802, Aug 2001. Page 9.

J. L. Rogers and R. P. Kesner. Lesions of the dorsal hippocampus or parietal cortex differentially affect spatial information processing. Behav Neurosci, 120(4):852-860, Aug 2006. Page 2.

J. Rosenfeld, J. Capdevielle, J. C. Guillemot, and P. Ferrara. In-gel digestion of proteins for internal sequence analysis after one- or two-dimensional gel electrophoresis. Anal Biochem, 203(1):173-179, May 1992. Page 31.

G. Rumbaugh, G.-M. Sia, C. C. Garner, and R. L. Huganir. Synapse-associated protein97 isoform-specific regulation of surface AMPA receptors and synaptic function in cultured neurons. J Neurosci, 23(11):4567-4576, Jun 2003. Page 8, 9, 10, 40, 100.

R. K. Saiki, D. H. Gelfand, S. Stoffel, S. J. Scharf, R. Higuchi, G. T. Horn, K. B. Mullis, and H. A. Erlich. Primer-directed enzymatic amplification of DNA with a thermostable DNA polymerase. Science, 239(4839):487-491, Jan 1988. Page 16.

J. Sambrook. Molecular Cloning: A Laboratory Manual, Third Edition (3 Volume Set). Cold Spring Harbor Laboratory Press, 2001. ISBN 0879695773. Page 13.

N. Sans, R. S. Petralia, Y. X. Wang, J. Blahos, J. W. Hell, and R. J. Wenthold. A developmental change in NMDA receptor-associated proteins at hippocampal synapses. J Neurosci, 20(3):1260-1271, Feb 2000. Page 35, 37, 48, 96. 


\section{REFERENCES}

N. Sans, C. Racca, R. S. Petralia, Y. X. Wang, J. McCallum, and R. J. Wenthold. Synapse-associated protein 97 selectively associates with a subset of AMPA receptors early in their biosynthetic pathway. J Neurosci, 21(19):7506-7516, Oct 2001. Page 10.

Y. Sato, Y-X. Tao, Q. Su, and R. A. Johns. Post-synaptic density-93 mediates tyrosinephosphorylation of the N-methyl-d-aspartate receptors. Neuroscience, Mar 2008. Page 66.

O. M. Schlüter, W. Xu, and R. C. Malenka. Alternative N-terminal domains of PSD-95 and SAP97 govern activity-dependent regulation of synaptic AMPA receptor function. Neuron, 51(1):99-111, Jul 2006. Page 7, 9, 10, 11, 13, 17, 18, 19, 37, 48, 49, $72,74,77,82,83,86,88,90,91,94,95,96,97,98,99$.

E. Schnell, M. Sizemore, S. Karimzadegan, L. Chen, D. S. Bredt, and R. A. Nicoll. Direct interactions between PSD-95 and stargazin control synaptic AMPA receptor number. Proc Natl Acad Sci U S A, 99(21):13902-13907, Oct 2002. Page 9, 10, 74, 95.

A. L. Shapiro, E. Viuela, and J. V. Maizel. Molecular weight estimation of polypeptide chains by electrophoresis in SDS-polyacrylamide gels. Biochem Biophys Res Commun, 28(5):815-820, Sep 1967. Page 28.

G. M. Shepherd, editor. The Synaptic Organization of the Brain. Oxford University Press, USA, 5 edition, 11 2003. ISBN 9780195159561. Page 3.

J. D. Shepherd and R. L. Huganir. The cell biology of synaptic plasticity: AMPA receptor trafficking. Annu Rev Cell Dev Biol, 23:613-643, 2007. Page 6.

A. Shevchenko, M. Wilm, O. Vorm, and M. Mann. Mass spectrometric sequencing of proteins silver-stained polyacrylamide gels. Anal Chem, 68(5):850-858, Mar 1996. Page 31 .

S. Shi, Y. Hayashi, J. A. Esteban, and R. Malinow. Subunit-specific rules governing AMPA receptor trafficking to synapses in hippocampal pyramidal neurons. Cell, 105 (3):331-343, May 2001. Page 5, 9. 
A. De Simoni, C. B. Griesinger, and F. A. Edwards. Development of rat CA1 neurones in acute versus organotypic slices: role of experience in synaptic morphology and activity. J Physiol, 550(Pt 1):135-147, Jul 2003. Page 2.

D. Siolas, C. Lerner, J. Burchard, W. Ge, P. S. Linsley, P. J. Paddison, G. J. Hannon, and M. A. Cleary. Synthetic shRNAs as potent RNAi triggers. Nat Biotechnol, 23 (2):227-231, Feb 2005. Page 44.

P. K. Smith, R. I. Krohn, G. T. Hermanson, A. K. Mallia, F. H. Gartner, M. D. Provenzano, E. K. Fujimoto, N. M. Goeke, B. J. Olson, and D. C. Klenk. Measurement of protein using bicinchoninic acid. Anal Biochem, 150(1):76-85, Oct 1985. Page 28.

V. Stein, D. R. C. House, D. S. Bredt, and R. A. Nicoll. Postsynaptic density-95 mimics and occludes hippocampal long-term potentiation and enhances long-term depression. J Neurosci, 23(13):5503-5506, Jul 2003. Page 9.

L. Stoppini, P. A. Buchs, and D. Muller. A simple method for organotypic cultures of nervous tissue. J Neurosci Methods, 37(2):173-182, Apr 1991. Page 2, 23.

G. R. Strichartz. The inhibition of sodium currents in myelinated nerve by quaternary derivatives of lidocaine. J Gen Physiol, 62(1):37-57, Jul 1973. Page 97.

M. E. Swartz, J. Eberhart, E. B. Pasquale, and C. E. Krull. EphA4/ephrin-A5 interactions in muscle precursor cell migration in the avian forelimb. Development, 128 (23):4669-4680, Dec 2001. Page 18.

M. J. Talbot and R. J. Sayer. Intracellular QX-314 inhibits calcium currents in hippocampal CA1 pyramidal neurons. J Neurophysiol, 76(3):2120-2124, Sep 1996. Page 97.

Y.-X. Tao, G. Rumbaugh, G.-D. Wang, R. S. Petralia, C. Zhao, F. W. Kauer, F. Tao, M. Zhuo, R. J. Wenthold, S. N. Raja, R. L. Huganir, D. S. Bredt, and R. A. Johns. Impaired NMDA receptor-mediated postsynaptic function and blunted NMDA receptordependent persistent pain in mice lacking postsynaptic density-93 protein. $\mathrm{J} \mathrm{Neu-}$ rosci, 23(17):6703-6712, Jul 2003. Page 10, 91, 98. 


\section{REFERENCES}

J. R. Topinka and D. S. Bredt. N-terminal palmitoylation of PSD-95 regulates association with cell membranes and interaction with K+ channel Kv1.4. Neuron, 20(1): 125-134, Jan 1998. Page 7.

H. Towbin, T. Staehelin, and J. Gordon. Electrophoretic transfer of proteins from polyacrylamide gels to nitrocellulose sheets: procedure and some applications. Proc Natl Acad Sci U S A, 76(9):4350-4354, Sep 1979. Page 28.

T. Umahara, T. Uchihara, A. Nakamura, and T. Iwamoto. Isoform-dependent immunolocalization of 14-3-3 proteins in developing rat cerebellum. Brain Res, 1253: 15-26, Feb 2009. Page 35.

UniProt Consortium. The Universal Protein Resource (UniProt) in 2010. Nucleic Acids Res, 38(Database issue):D142-D148, Jan 2010. Page 34, 64.

C. L. Waites, C. G. Specht, K. Hrtel, S. Leal-Ortiz, D. Genoux, D. Li, R. C. Drisdel, O. Jeyifous, J. E. Cheyne, W. N. Green, J. M. Montgomery, and C. C. Garner. Synaptic SAP97 isoforms regulate AMPA receptor dynamics and access to presynaptic glutamate. J Neurosci, 29(14):4332-4345, Apr 2009. Page 8, 10, 95.

C.-Y. Wang, K. Chang, R. S. Petralia, Y.-X. Wang, G. K. Seabold, and R. J. Wenthold. A novel family of adhesion-like molecules that interacts with the NMDA receptor. $J$ Neurosci, 26(8):2174-2183, Feb 2006. Page 35.

R. J. Wenthold, R. S. Petralia, I. I. Blahos J, and A. S. Niedzielski. Evidence for multiple AMPA receptor complexes in hippocampal CA1/CA2 neurons. J Neurosci, 16(6):1982-1989, Mar 1996. Page 5.

R. J. Wenthold, K. Prybylowski, S. Standley, N. Sans, and R. S. Petralia. Trafficking of NMDA receptors. Annu Rev Pharmacol Toxicol, 43:335-358, 2003. Page 4.

A. Wenzel, J. M. Fritschy, H. Mohler, and D. Benke. NMDA receptor heterogeneity during postnatal development of the rat brain: differential expression of the NR2A, NR2B, and NR2C subunit proteins. J Neurochem, 68(2):469-478, Feb 1997. Page 4.

W. Xu, O. M. Schlüter, P. Steiner, B. L. Czervionke, B. Sabatini, and R. C. Malenka. Molecular Dissociation of the Role of PSD-95 in Regulating Synaptic Strength and LTD. Neuron, 57(2):248-262, Jan 2008. Page 7, 52, 56, 58, 91, 96. 
Y. Yang, X. Wang, M. Frerking, and Q. Zhou. Delivery of AMPA receptors to perisynaptic sites precedes the full expression of long-term potentiation. Proc Natl Acad Sci U S A, 105(32):11388-11393, Aug 2008. Page 6.

R. S. Zucker and W. G. Regehr. Short-term synaptic plasticity. Annu Rev Physiol, 64: 355-405, 2002. Page 74. 


\section{Curriculum Vitae}

Personal Data

Name:

Juliane Marie Krüger, formerly Gansert

Address:

Weender Str. 86

37073 Göttingen

E-mail: mail@juliane-krueger.com

Date and place of birth: May $8^{\text {th }}, 1983$ in Lüdenscheid

Education

01/2007 - 06/2010 PhD with Dr. Dr. Oliver Schlüter

European Neuroscience Institute Göttingen, Germany

Thesis: Expression and function of PSD-93 isoforms in hippocampal synapses.

09/2005 - 05/2006 M.Sc. in Computational Biology (GPA: 3.88/4.0)

New Jersey Institute of Technology, Newark, NJ, USA

Thesis: Network activity arising from optimal diameters of neuronal processes.

10/2002 - 07/2005 B.Sc. in Computational Life Science (Final grade: 1.4) University of Lübeck, Germany

Thesis: Medical image registration using a multigrid approach.

06/2002 High School Diploma (Abitur, final grade: 1.1)

Evangelisches Gymnasium Meinerzhagen, Germany

Relevant Work Experience and Internships

06/2006 - 08/2006 New Jersey Institute of Technology, Full-time Research Associate

12/2004 - 06/2005 Drägerwerk AG, Basic research and development, Student Trainee

09/2003 - 10/2003 Institute of Medicine, Research Center Jülich, Intern 
Courses and Workshops

09/2009 European Synapse Summer School, Bordeaux, France

09/2008 Course on High Resolution Microscopy

Scholarships

09/2005 - 05/2006 New Jersey Institute of Technology Provost Scholarship

08/2005 - 05/2006 Fulbright Scholarship

Publications

PEer-ReVieWed PAPERS

Gansert J, Golowasch J and Nadim F: Sustained rhythmic activity in gap-junctionally coupled networks of model neurons depends on the diameter of coupled dendrites. J Neurophysiol, 2007.

\section{Conference Posters}

Gansert $\mathbf{J}$ and Schlüter OM: Expression and function of PSD-93 isoforms in hippocampal synapses. EU Synapse Meeting, 2009.

Nadim F, Gansert J and Golowasch J: Activity in gap-junctionally coupled networks depends on the diameter of coupled processes. Society for Neuroscience Annual Meeting 32: 634.24, 2006. 